Daniel de la Riva Massaad

\title{
An invitation to Noise Sensitivity and applications to quenched Voronoi Percolation
}

Thesis presented to the Programa de Pós-graduação em Matemática, do Departamento de Matemática da PUC-Rio in partial fulfillment of the requirements for the degree of Mestre em Matemática.

Advisor: Prof. Simon Griffiths 


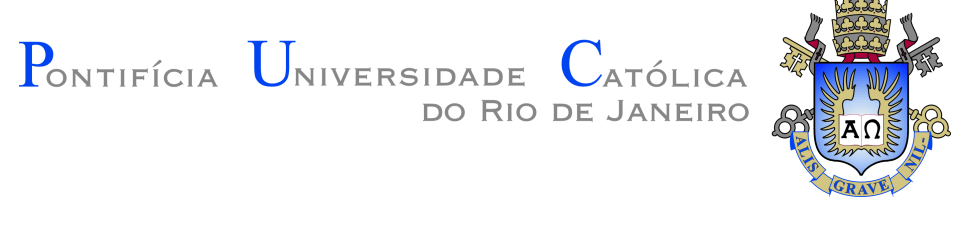

Daniel de la Riva Massaad

\begin{abstract}
An invitation to Noise Sensitivity and applications to quenched Voronoi Percolation
\end{abstract}

Thesis presented to the Programa de Pós-graduação em Matemática da PUC-Rio in partial fulfillment of the requirements for the degree of Mestre em Matemática. Approved by the Examination Committee:

Prof. Simon Griffiths

Advisor

Departamento de Matemática - PUC-Rio

Prof. Roberto Imbuzeiro Moraes Felinto de Oliveira Instituto de Matemática Pura e Aplicada - IMPA

Prof. Boyan Slavchev Sirakov Departamento de Matemática - PUC-Rio

Prof. Augusto Quadros Teixeira Instituto de Matemática Pura e Aplicada - IMPA

Prof. Robert David Morris Instituto de Matemática Pura e Aplicada - IMPA 
All rights reserved.

\section{Daniel de la Riva Massaad}

Majored in mathematics by Pontifícia Universidade Católica do Rio de Janeiro (Rio de Janeiro, Brazil)

Bibliographic data

de la Riva Massaad, Daniel

An invitation to Noise Sensitivity and applications to quenched Voronoi Percolation / Daniel de la Riva Massaad; advisor: Simon Griffiths. - 2020.

111 f: il. color. ; $30 \mathrm{~cm}$

Dissertação (mestrado) - Pontifícia Universidade Católica do Rio de Janeiro, Departamento de Matemática, 2020.

Inclui bibliografia

1. Matemática - Teses. 2. Probabilidade - Teses. 3. Sensibilidade a Ruído. 4. Martingal. 5. Análise de Fourier. 6. Função Booleana. 7. Percolação. 8. Desigualdade de Efron-Stein. 9. Quenched . I. Griffiths, Simon. II. Pontifícia Universidade Católica do Rio de Janeiro. Departamento de Matemática. III. Título. 
To all the professors and thinkers that shaped my thoughts 


\section{Acknowledgments}

I would like to begin this by thanking my parents. My mother, Marcia, always supported me to pursue my mathematical studies, with love and care. My father, Ricardo, taught me the discipline of a warrior, required for the ones that aim at greatness.

My friends from IMPA, Thomás, Daniel, Caio, Luiz and Guilherme that were great study partners and gave me great advices.

My friends from PUC's mathematics department, Adailton, Paulo, Marcelo, Matheus, Leonardo, José, Thiago, Aline, Hugo, Byron, Luize, Caetano, Gabriel, Victor, Igor, David, Filipe and Joao that helped me with a large number of courses.

My friends from PUC's logic department, German and Victor introduced me to the study of Logics, one of the most important and beautiful fields of mathematics.

To my childhood friends, Pedro and Antonio.

To my friends from Iguape and members of the Camus' fan club, Cadu, Julia, Matheus and Remo.

To my friends from Toca de Assis, specially Cris, Damião, Carol, Rosa, Edson, and all our brothers.

This study was financed in part by the Coordenação de Aperfeiçoamento de Pessoal de Nível Superior - Brasil (CAPES) - Finance Code 001, and FAPERJ.

I would also like to thank all the professores that helped me throughout my life. Specially:

Professor Luiz Carlos, from PUC's logic department, that presented amazing seminars with the enthusiasm of a passionate professor.

Professors Cadu, Gabriel, and Remo, that bravely endured my long divagations and taught me the most important subject developed by mankind: Philosophy.

Professor Daniel Ahlberg for sharing his ideas and trusting me to work with them. Without his aid it wouldn't be possible for me to make the improvements listed in this thesis.

And finally, Professor Simon Griffiths. More than an advisor, Simon was a friend to me. With patience and respect he brought back my desire for mathematics. If one day I will be called a mathematician, it is all due to Simon and the opportunities he gave me. 


\section{Abstract}

de la Riva Massaad, Daniel; Griffiths, Simon (Advisor). An invitation to Noise Sensitivity and applications to quenched Voronoi Percolation. Rio de Janeiro, 2020. 111p. Dissertação de Mestrado - Departamento de Matemática, Pontifícia Universidade Católica do Rio de Janeiro.

We begin this dissertation by giving an introductory overview of the topics of Noise Sensitivity and Percolation. As these areas may be unfamiliar to many graduate students, we present the material in an accessible way, with the objective of publicising important techniques and results in these areas. We shall also introduce the model of Voronoi Percolation and present results of Vincent Tassion on crossing probabilities in this model. In the last two chapters we consider Noise Sensitivity of Quenched Voronoi Percolation. In particular, in the penultimate chapter we present the results of the paper "Quenched Voronoi Percolation" by Daniel Ahlberg, Simon Griffiths, Robert Morris and Vincent Tassion, and in the final chapter we prove a theorem which improves one of the bounds of that paper.

\section{Keywords}

Noise Sensitivity; Martingale; Fourier Analysis; Boolean Function; Percolation; Efron-Stein Inequality; Quenched. 


\section{Resumo}

de la Riva Massaad, Daniel; Griffiths, Simon. Um convite à "Sensibilidade a Ruído" e aplicações para Percolação de Voronoi do tipo quenched. Rio de Janeiro, 2020. 111p. Dissertação de Mestrado - Departamento de Matemática, Pontifícia Universidade Católica do Rio de Janeiro.

Nós começamos essa dissertação com um panorama geral e introdutório dos tópicos de "Sensibilidade a Ruído" e "Percolação" . Como essas áreas podem ser desconhecidas por muitos estudantes de pós-graduação, nós apresentamos o material de uma maneira acessível, com o intuito de divulgar importantes técnicas e resultados dessas áreas. Nós também vamos introduzir o modelo para Percolação de Voronoi e apresentar resultados sobre probabilidades de cruzamentos neste modelo. Nos últimos dois capulos nós iremos considerar Sensibilidade a Ruído para Percolação do tipo "quenched". Em particular, no penúltimo capítulo nós vamos apresentar resultados do artigo "Quenched Voronoi Percolation" de Daniel Ahlberg, Simon Griffiths, Robert Morris e Vincent Tassion, e no último capítulo provaremos um teorema que melhora uma das cotas deste artigo.

\section{Palavras-chave}

Sensibilidade a Ruído; Martingal; Análise de Fourier; Função Booleana; Percolação; Desigualdade de Efron-Stein; Quenched. 


\section{Table of contents}

1 Introduction 10

2 Basic Tools $\quad 15$

$\begin{array}{ll}2.1 \text { Martingale } & 15\end{array}$

$\begin{array}{lll}2.2 & \text { Boolean Functions } & 16\end{array}$

2.3 Fourier Analysis on the Hypercube 19

$3 \quad$ Percolation on $\mathbb{Z}^{2} \quad 22$

3.1 Definition of the model and other basic properties. 22

3.2 Russo-Seymour-Welsh 26

3.3 Margulis-Russo Formula 29

3.4 The critical point $p_{c}=1 / 2 \quad 31$

4 Voronoi Percolation on $\mathbb{R}^{2} \quad 36$

4.1 Definition of the model 37

4.2 Russo-Seymour-Welsh for Voronoi Percolation in the plane 38

$5 \quad$ Noise Sensitivity of Boolean Functions 48

$5.1 \quad$ Influence 49

5.2 Noise Sensitivity 52

5.3 The Benjamini-Kalai-Schramm Noise Sensitivity Theorem 55

5.4 The Steif-Schramm Revealment Theorem 57

$\begin{array}{lll}5.5 & \text { Crossings are Noise Sensitive } & 60\end{array}$

6 Quenched Voronoi Percolation $\quad 62$

6.1 Two more Poisson models (to deal with boundary problems) 64

6.2 Weak bounds for crossing probabilities on Quenched Voronoi Percolation 65

6.3 A bound on the probability of the 1-arm event 68

6.4 Variance and Influence 74

6.5 Noise Sensitivity of crossings in Quenched Voronoi Percolation 75

7 On the rate of convergence in Quenched Voronoi Percolation $\quad \mathbf{8 2}$

$\begin{array}{lll}7.1 & \text { Relations between the models } & 85\end{array}$

7.2 Improved bound for the sum of squares of the Influences 86

7.3 Improved bound on the crossing probabilities for Voronoi Percolation 90

7.4 Proof of the Main Theorem for the auxiliary models 95

7.5 Final remarks to achieve the improvement on the original model 100

$\begin{array}{ll}\text { Bibliography } & 104\end{array}$

$\begin{array}{ll}\text { A Classic Inequalities } & 106\end{array}$ 
Il n'y a pas d'amour de vivre sans désespoir de vivre.

Albert Camus, Entre Oui et Non. 


\section{Introduction}

When I was an undergraduate student I often found it quite hard to understand the titles of dissertations and seminars. Not only were they long and unattractive, often they were completely incomprehensible.

Even now, as a graduate student, I still get confused by these titles. And it was not long until I noticed that my first work, my master's thesis, has an extremely long, unattractive, and incomprehensible title.

But if I want to be sincere with my former self, my first goal is to make this title more comprehensible for any math student that desires to read this text. Let us, then, begin our explanation.

What is Percolation? From the Latin word "percolare", percolation refers to the movement and filtering of fluids through porous materials. From a mathematical point of view, it was first introduced in the article "Percolation process", by Broadbent and Hammersley [6]. This paper studied in a general way how the random properties of a "medium" influence the percolation of a "fluid" through it, for example, water penetrating a porous stone.

As usual, they formalized this model and turned it into a problem that can be analyzed from a mathematical perspective. Suppose that a lump of porous material is placed in a bucket of water, and we wish to know how much of the interior becomes wet. Suppose also that we can represent the pore system of the material as a maze, in which the water is able to flow through sufficiently large pores to the interstices of the interior. Consider that each possible "tunnel" is open independently with probability $p$ (for some $p \in[0,1]$ ) and water may pass through open tunnels. Will the water reach a point near the center of the stone? For how many paths will this happen? And what proportion of a porous stone is wetted? And from that, the first percolation model was born. Instead of imagining stones, we may take rectangles formed by the grid of $\mathbb{Z}^{2}$ and, instead of imagining channels, we may consider the edges of $\mathbb{Z}^{2}$ and assign independently for each one of them, probability $p$ of being "open" and $1-p$ of being "closed". We include the following image from [10]. 


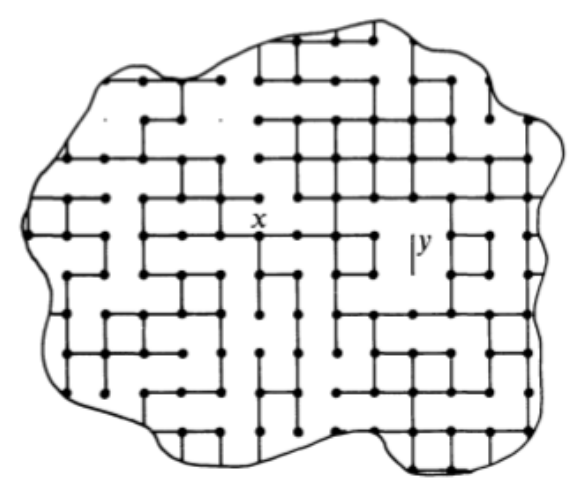

Figure 1.1: The contour represents the boundary of the stone, the black edges represent the open tunnels and the empty edges represent the closed tunnels. Note that $x$ is wetted but $y$ is not.

The probability of wetting points near the center for large-scale stones is related to the existence of infinite connected "clusters" in $\mathbb{Z}^{2}$. We can see this by observing if a center point is wetted, there must be an open path from the boundary of the stone to it. If we take larger structures, more tunnels will appear and the grid will get bigger. So, it is natural to study the existence of such components since they will imply those events for any size of stones. One may note that for $p=0$ there is no edge and for $p=1$ we have all edges of $\mathbb{Z}^{2}$. So what is happening with the intermediate probabilities? Is there a sort of critical point which changes the behavior of this process? These questions will be addressed in Chapter 3, together with a more formal definition of our model.

Now that we know the meaning of Percolation, and specifically, Percolation on $\mathbb{Z}^{2}$, we may wonder what is Quenched Voronoi Percolation. But first, we must study the meaning of Voronoi Percolation.

In Voronoi Percolation, we still want to analyze properties such as the existence of infinite connected components, but instead of working with $\mathbb{Z}^{2}$, we will work with $\mathbb{R}^{2}$. Which types of crossings will we be looking for now? To introduce a natural model in $\mathbb{R}^{2}$ we will define the notion of Voronoi Tessellation informally.

First developed by the mathematician Georges Voronoi in the article "Nouvelles applications des parametres continus a la theorie des formes quadratiques. Premier memoire. Sur quelques proprietes des formes quadratiques positives parfaites" from 1908 [19], a Voronoi Tessellation looks at chosen points ( in our scenario, often chosen in a random way ) in a rectangle and define their territory by the region closest to each point. This territory will be named a "tile", and will also look like one. One can also extend the Tessellation to 
the whole plane, by choosing points with random processes in $\mathbb{R}^{2}$, such as the Poisson Point Process.

Now imagine that and my advisor Simon and I are playing a war game. First, Matheus (one of Simon's students) will pick up, let's say $n=10$ points, and distribute them in a random way across the whiteboard. Afterwards, Simon and I will try to conquer each territory. Since we are both fair people, a coin will be flipped for each tile and be given color red when it flips heads (open), and blue when it flips tails (closed). However, maybe Simon is not so fair as I thought and rigs up the coin, in such a way that the probability of landing heads will be now $0 \leq p \leq 1$. We can see that a very similar problem arises. Will there be a way to cross from the boundary of the whiteboard to the middle of it in red? Or maybe a path that uses only red tiles from the left to the right side of the rectangle? Again, it is trivially not possible for $p=0$ and trivially possible for $p=1$. But, since the tiles now have different sizes and shapes, our new structure is much more uncertain. Will we be able to achieve similar properties to $\mathbb{Z}^{2}$ ? In Chapter 4 the model will be properly introduced and some of these questions will be answered, mainly based on the work of Vincent Tassion "Crossing probabilities for Voronoi percolation" [18].

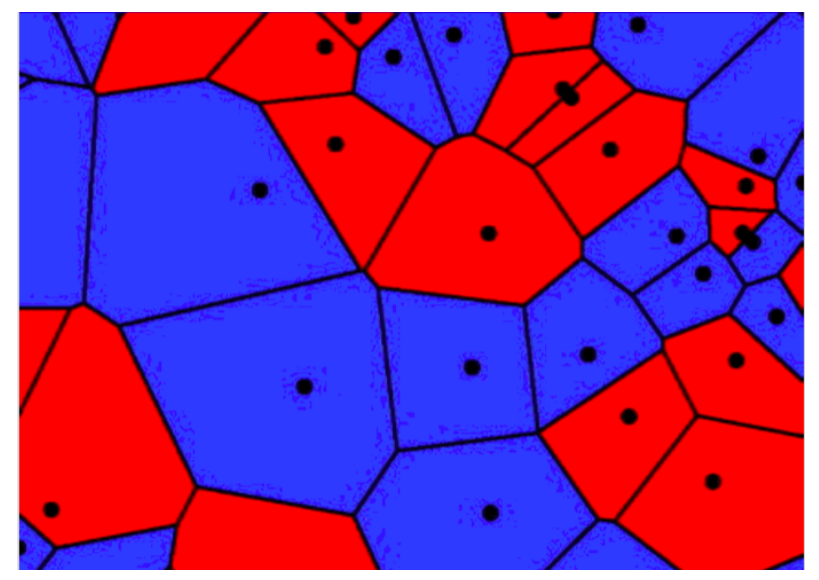

Figure 1.2: Here we look at an excerpt of the Voronoi Percolation Process in $\mathbb{R}^{2}$. The image was take from the cover of [4].

Now let's explain the "Quenched" part. By "Quenched Voronoi Percolation" we mean a Voronoi Percolation processes (as described above), in which we have already know (and have fixed) the tiles. The only thing yet to be revealed is the color of each tile. One can note that now, the probability of some events will depend on the model thus generated, since, for example, the position of the points alters the probability of a left to right crossing. We finally reach our last complicated expression. What is Noise Sensitivity and how does it relate to Quenched Voronoi Percolation? 
In contrast with our previous notions, this is an surprisingly recent idea. It was first introduced in 1999 in the seminal paper by Benjamini, Kalai, and Schramm "Noise Sensitivity of Boolean Functions and applications to Percolation" [2]. This is a more refined concept and in order to illustrate it, we will give the example of an election. Let us say that Simon and his students will decide who is the funniest, Matheus or Daniel. Note that if, apart from Matheus and Daniel, Simon and his students form a group of 11 people this election may be characterized by a so-called Boolean function:

$$
f:\{0,1\}^{11} \rightarrow\{0,1\}
$$

where 0 represents the votes for Matheus and 1 represents the votes for Daniel. The outcome of this function is the winner.

But as you may have noticed, there can be many possible ways to organize this election. Maybe it will be the "fairest" system, which is the democracy: The one who receives the highest number of votes wins! Maybe Simon is a sort of Dictator, and only his vote counts. Or maybe if only one person votes for Matheus, he is automatically the winner.

Now imagine that Igor, an outsider, and a former student of Simon, arrives, and with probability $\varepsilon>0$ chooses each person and alters their vote. Will the outcome easily change or is it a little bit more stable than Igor thought?

Noise Sensitive functions, as the name suggests, are the ones that will easily change their outcome with small disturbances. So, in those cases, knowing most of the information still doesn't help us a lot in predicting the outcome. In our example, Quenched Voronoi Percolation, with $p=1 / 2$ (which is particularly interesting as it is the critical probability), the event of a red leftto-right crossing in a rectangle will be asymptotically noise sensitive. That is, in a large rectangle, with a given tiling and coloring, if a small proportion of colors are changed (at random) we have almost no information about whether the crossing will occur. The definitions and classical theorems of Noise Sensitivity are presented in Chapter 5 .

We now give an overview of the dissertation. After Chapter 2, in which we introduce basic tools and concepts, we present an account of major results in the areas of Percolation on $\mathbb{Z}^{2}$ (Chapter 3), Voronoi Percolation on $\mathbb{R}^{2}$ (Chapter 4) and Noise Sensitivity (Chapter 5). Our hope is that these Chapters will be accessible to interested students.

We then turn to Quenched Voronoi Percolation in Chapter 6. We will discuss results from the paper "Quenched Voronoi Percolation", by Ahlberg, 
Griffiths, Morris, and Tassion [1] in 2015. At some moments in that paper there are important details which are left a little vague. This does not represent a major problem, however, and we explain how these arguments can be made completely rigorous.

The authors proved a major conjecture of [2]. Essentially, the conjecture states that knowing the Voronoi Tessellation, but not knowing the colors of the cells, gives almost no information whether or not a red horizontal crossing exists.

Finally, in Chapter 7, we shall present a new result (Theorem 7.1) which improves on the probability bound given by Theorem 1.1 of [1] (which appears here as Theorem 6.2). We remark that many of the ideas involved in the proof arose in discussions with Daniel Ahlberg and Simon Griffiths.

We cannot at this point give a full description of our approach. However, we may mention that it is based on the idea of proving subsequently stronger bounds at larger scales by iterating previous results. To make this approach rigorous we need to better understand the relation between different models and make some other changes to the proofs.

We also state and prove some classic inequalities that were used throughout this thesis in the Appendix. 


\section{Basic Tools}

In this Chapter we present the basic tools that we will throughout this dissertation. We present Martingales in Section 2.1, Boolean functions in Section 2.2 and Fourier Analysis on the hypercube in Section 2.3.

\section{1}

\section{Martingale}

In this Section we will define the concepts of conditional expectation and Martingale. Since this is a very well known idea, and it is often presented in basic probability courses, we will only list the properties that we will use and refer to [7] for proofs and details.

Definição 2.1 Given a probability space $\left(\Omega, \mathcal{F}_{0}, P\right)$, a $\sigma$-field $\mathcal{F} \subseteq \mathcal{F}_{0}$, and a random variable $X \in \mathcal{F}_{0}$ with $E[|X|]<\infty$, we define the conditional expectation of $X$ given $\mathcal{F}, E(X \mid \mathcal{F})$ to be any random variable $Y$, such that

1. $Y \in \mathcal{F}$, i.e., $Y$ is $\mathcal{F}$-measurable.

2. For all $A \in \mathcal{F}, \int_{A} X d P=\int_{A} Y d P$.

We can actually verify that $Y$ always exists and $Y$ is unique.

We now list a series of properties that will be used.

Theorem 2.2 Assume $E[|X|], E[|Y|]<\infty$. Then:

1. $E(a X+Y \mid \mathcal{F})=a E(X \mid \mathcal{F})+E(Y \mid \mathcal{F})$.

2. If $X \leq Y$, then $E(X \mid \mathcal{F}) \leq E(Y \mid \mathcal{F})$.

3. If $X$ is independent of $\mathbb{1}_{A}$, for every $A \in \mathcal{F}$, then $E(X \mid \mathcal{F})=E(X)$

4. If $X$ is $\mathcal{F}$-measurable, then $E(X \mid \mathcal{F})=X$.

5. If $X$ is $\mathcal{F}$-measurable, then $E(X Y \mid \mathcal{F})=X E(Y \mid \mathcal{F})$.

6. We have the Law of total expectation: $E(E(X \mid \mathcal{F}))=E(X)$.

7. Tower property: If $\mathcal{F}_{1} \subseteq \mathcal{F}_{2} \subseteq \mathcal{F}_{0}$, we have $E\left(E\left(X \mid \mathcal{F}_{2}\right) \mid \mathcal{F}_{1}\right)=E\left(X \mid \mathcal{F}_{1}\right)$. 
Now that we have listed those properties, we can define a martingale.

Definição 2.3 Let $\mathcal{F}_{n}$ be a filtration, i.e., an increasing sequence of $\sigma$-fields. $A$ sequence $X_{n}$ is said to be adapted to $\mathcal{F}_{n}$ if $X_{n} \in \mathcal{F}_{n}$ for all $n$. If $X_{n}$ is a sequence with

1. $E\left[\left|X_{n}\right|\right]<\infty$.

2. $X_{n}$ is adapted to $\mathcal{F}_{n}$.

3. $E\left(X_{n+1} \mid \mathcal{F}_{n}\right)=X_{n}$ for all $n$,

Then, $X$ is said to be a martingale with respect to $\mathcal{F}_{n}$. If in the last definition, $=$ is replaced by $\leq$ or $\geq$, then $X$ is said to be a supermartingale or submartingale, respectively.

Theorem 2.4 (Martingale Convergence Theorem) If $X_{n}$ is a submartingale with $\sup _{n} E\left[X_{n}^{+}\right]<\infty$, then as $n$ approaches infinity, $X_{n}$ converges almost surely to a limit $X$ with $E[|X|]<\infty$.

We remark that there are many interesting regarding martingales, butt we won't need them in this thesis. We, one more time, refer the interested reader to [7].

\section{2}

\section{Boolean Functions}

The following Sections will be largely based on the definitions and examples defined in [9].

The concept of Boolean functions is extremely important in many areas such as Computer Science and Logic. As the name suggests, a Boolean function is a function whose argument and outcome gets assigned with two possible values, often interpreted as False Value and Truth Value. In the introduction, this concept was stated in an informal way and it was directly related to the idea of an election. More generally:

Definição 2.5 A Boolean function is a function from the Hypercube $\Omega_{n}:=$ $\{-1,1\}^{n}$ into either $\{0,1\}$ or $\{-1,1\}$.

The Hypercube is more often defined on $\{0,1\}^{n}$. But for Fourier Analysis on the Hypercube, developed in the next Section, using $\Omega_{n}=\{-1,1\}^{n}$ is more convenient, and since there is no fundamental difference between both of them, we will stick with the latter choice.

Sometimes it will be more convenient to work with functions with image in $\{-1,1\}$, while at other times $\{0,1\}$ is preferable. Again, there is no essential 
difference between them since all the theorems stated for Boolean functions can be attained for both.

In some cases we may even consider $f: \Omega_{n} \rightarrow \mathbb{R}$, which is not a Boolean function.

Since we will be interested in working in a probabilistic setting, the Hypercube may be endowed with the uniform measure $\mathbb{P}$, where if $w:=$ $w_{n}=\left(w_{1}, \ldots, w_{n}\right) \in \Omega_{n}$, and $X$ is the discrete uniform random variable, then $\mathbb{P}(X=w)=1 / 2^{n}$ for every $w \in \Omega_{n}$ and $\mathbb{E}$ denotes the corresponding expectation.

Occasionally, $\Omega_{n}$ will be endowed with the weighted product measure $P_{p}(X=w)=p^{\left|W_{-1}\right|}(1-p)^{\left|W_{1}\right|}$, where $\left|W_{-1}\right|$ represents the number of $-1^{\prime} s$ in $w$, and $\left|W_{1}\right|$ represents the number of $1^{\prime} s$ in $w . \mathbb{E}_{p}$ will denote the corresponding expected value.

Another useful concept will be that of monotone functions. For vectors, $x$ and $y$, we write $x \leq y$ if $x_{i} \leq y_{i}$ for all $i$.

Definição 2.6 A function $f$ is monotone, if $f(x) \leq f(y)$ whenever $x \leq y$.

This is an important class of functions since they have a number of interesting properties, and some theorems, will be valid just for them.

We will now introduce three basic examples that will have a number of interesting properties. In particular, we may consider the notion of influence which is related to changing the outcome of the function. A formal definition will be given in Chapter 3. We will use the language of an election to be more intuitive to the readers new to this area. We also encourage them to verify which of these functions are monotone.

\section{Example 2.7 (Dictator)}

$$
\operatorname{Dict}_{n}\left(x_{1}, \ldots, x_{n}\right):=x_{1} \text {. }
$$

As you expect from a dictator, the first "voter" determines the outcome of this function. One may note that only the first bit has influence over the election.

\section{Example 2.8 (Parity)}

$$
\operatorname{Par}_{n}\left(x_{1}, \ldots, x_{n}\right):=\prod_{i=1}^{n} x_{i} .
$$

The outcome of this function is determined by whether the number of $-1^{\prime}$ s in $w$ is even or odd. In this example every "voter" has the chance to change the outcome of this function. So, all of them have total influence. 
Example 2.9 (Majority) Let $n$ be an odd number and define

$$
\operatorname{Maj}_{n}\left(x_{1}, \ldots, x_{n}\right):=\operatorname{sign}\left(\sum_{i=1}^{n} x_{i}\right) .
$$

This function exemplifies the idea of a democracy. We can see that all the "voters" have the same influence, and a "voter" decides the fate of the election only when the sum of the other votes is zero.

What would be the influence of each voter in this example? To even contemplate this question would require a more formal definition of influence. The enthusiastic reader may try to think of a possible definition that would correspond to our intuition. See Chapter 3 for details.

We now give two more complicated examples:

Example 2.10 (Tribes) Partition $[n]=(1, \ldots, n)$ into disjoint blocks of length $\log _{2}(n)-\log _{2}\left(\log _{2}(n)\right)$. Define $f_{n}$ to be 1 if there exists at least one block that contains only $1^{\prime} s$, and 0 otherwise. Note that this represents an election which must have an unanimity of $1^{\prime} s$, in at least one tribe, to elect 1 . We can see that for a "voter" to decide the outcome of the election, it must be in a tribe with only 1-voters and we can't have unanimity in any other tribe. Only in this case the "voter" will influence the result of the election. The choice of the length is exactly made to achieve an interesting result regarding such quantity.

Example 2.11 (Crossings in $\mathbb{Z}^{2}$ ) We start with the graph $\mathbb{Z}^{2}$ which has vertices being the set $\mathbb{Z}^{2}$ and edges between pairs of points at Euclidean distance 1. Consider $S_{n}:=[0, n] \times[0, n]$, to be the subgraph determined by such vertices. We may now, color each edge independently with probability $0 \leq p \leq 1$ for the color "red" (open), and $1-p$ for the color "blue" (closed). Define $H_{S_{n}}$ to be the event that there exists a red path through the edges from $\{0\} \times[0, n]$ to $\{n\} \times[0, n]$. We call it a horizontal red crossing. Define $f_{n}:=\mathbb{1}_{S_{n}}$, which represents the indicator of the even $S_{n}$. Understanding the influence of such events turns out to be an extremely interesting and difficult problem. The image below represents the 4-arm event, for a similar and more natural model. This is exactly the case of a decisive voter (when it is far from the boundary). We may also define Crossings for the Voronoi Tessellation in a similar way. This will be made more precise in Chapter 4. 


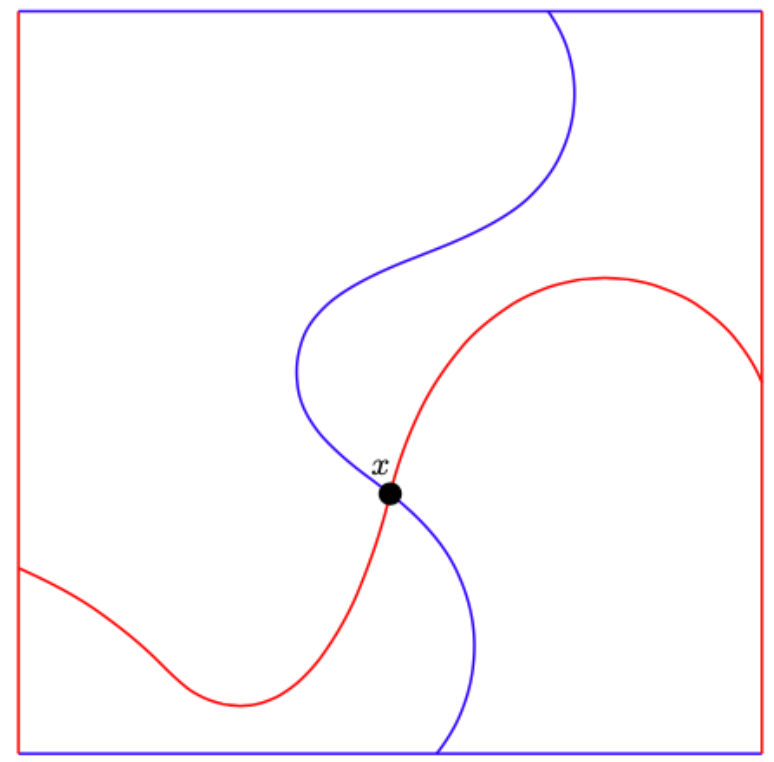

Figure 2.1: In this image we look at a Hex Board Game. It is a more natural environment to look at the 4-arm event. For those who are not familiar with this game, we take a board composed of Hexagons with two parallels red walls, and two parallels blue walls as in the drawing. Each player chooses a color and the winner is the one can get a crossing from one side to the other with the same color as the side. There can be only one winner, so if you make a drawing of the board you can note that a red crossing blocks a blue on and vice-versa. As it can be seen in the image the black node $x$ decides the winner of such game. Note that if it is colored blue, $f_{n}=0$ and if it is red $f_{n}=1$.

\section{3}

\section{Fourier Analysis on the Hypercube}

Another useful tool will be Fourier analysis on the hypercube. In this Section we will introduce some basic definitions and calculate some of the so-called Fourier-Walsh Coefficients for some of the previous examples.

It is natural, when applying Fourier Analysis, to consider the set $L^{2}\left(\{-1,1\}^{n}\right)$, of all real-valued functions on $\Omega_{n}$ endowed with the following inner product:

$$
\begin{aligned}
\langle f, g\rangle & :=\sum_{x_{1}, \ldots, x_{n}} 2^{-n} f\left(x_{1}, \ldots, x_{n}\right) g\left(x_{1}, \ldots, x_{n}\right) \\
& =\mathbb{E}[f g] \quad \text { for all } f, g \in L^{2}\left(\Omega_{n}\right)
\end{aligned}
$$

For any $S \subseteq\{1, \ldots, n\}$, let $\chi_{S}$ be a function on $\Omega_{n}$ defined by

$$
\chi_{S}(w):=\prod_{i \in S} x_{i}
$$

Observe that $\chi_{\emptyset} \equiv 1$. Also note that this family of $2^{n}$ elements forms an 
orthonormal basis for $\Omega_{n}$. To see this, first note that we have that for any $S$

$$
\left\langle\chi_{S}, \chi_{S}\right\rangle=\sum_{x_{1}, \ldots, x_{n}} 2^{-n} \prod_{x i \in S} x_{i}^{2}=1
$$

and that for $S \neq T$

$$
\left\langle\chi_{S}, \chi_{T}\right\rangle=2^{-n} \sum_{x_{1}, \ldots, x_{n}} \prod_{i \in S \triangle T} x_{i}=0
$$

by symmetry. Note that $\triangle$ represents the symmetric difference. Finally, observe that any $f \in L^{2}\left(\Omega_{n}\right)$ may be represented as

$$
f(x)=\sum_{a_{1}, \ldots, a_{n}} f(a) \mathbb{1}_{\{a\}}(x),
$$

with

$$
\mathbb{1}_{\{a\}}(x):=\left(\frac{1+a_{1} x_{1}}{2}\right) \ldots\left(\frac{1+a_{n} x_{n}}{2}\right) .
$$

Considering, then, the monomials $\chi_{S}$, we get that:

$$
f=\sum_{S \subseteq\{1, \ldots, n\}} \hat{f}(S) \chi_{S}
$$

and so it forms a basis. The $\hat{f}(S)$ are called the Fourier-Walsh coefficients of $f$. They also satisfy that

$$
\hat{f}(S):=\left\langle f, \chi_{S}\right\rangle=\mathbb{E}\left[f \chi_{S}\right]
$$

Because of that, we have that our representation is unique given the basis $\chi_{S}$. Note that $\hat{f}(\emptyset)=\mathbb{E}[f]$ and we have the Parseval's formula

$$
E\left[f^{2}\right]=\sum_{S \subseteq\{1, \ldots, n\}} \hat{f}^{2}(S)
$$

We define one more concept. We also remark that all of those concepts, that may seem slightly arbitrary, will be extremely useful in Chapter 5 .

Definição 2.12 For any function $f: \Omega_{n} \rightarrow \mathbb{R}$, the energy spectrum $E_{f} i$ defined by

$$
E_{f}(m):=\sum_{|S|=m} \hat{f}(S)^{2}, m \in\{1, \ldots, n\}
$$

We will now calculate the Fourier expansion of some of the previous examples.

Parity easily gives us that $f=\chi_{[n]}$, since this is exactly the definition of the function, and as we stated, the representation is unique. 
Dictator also easily gives the expansion directly by the definition of the function $f=\chi_{1}$.

Majority has a slightly more complicated expansion. First, note that if $|S|$ is even we have that $\widehat{M a j_{n}}(S)=0$, since $M a j_{n}$ is an odd function. For $|S|=k$ odd we refer to Theorem 5.19 of [14] which states that

$$
\widehat{\operatorname{Maj}_{n}}(S)=(-1)^{\frac{k-1}{2}} \frac{\left(\begin{array}{c}
\frac{n-1}{2} \\
\frac{k-1}{2}
\end{array}\right)}{\left(\begin{array}{l}
n-1 \\
k-1
\end{array}\right)} 2^{n-1}\left(\begin{array}{c}
n-1 \\
\frac{n-1}{2}
\end{array}\right) .
$$

With that in mind we end this introductory Section. Now that we have the most important tools, we can begin the most important ideas of this thesis. 


\section{Percolation on $\mathbb{Z}^{2}$}

Now we can finally focus on studying Percolation. We will introduce the first and most basic model of percolation: Percolation on $\mathbb{Z}^{2}$. In the introduction, we already mentioned how this model arose, and we gave an intuitive definition of it and asked the reader some questions regarding this model. Since there are plenty of texts regarding Percolation on $\mathbb{Z}^{2}$ we will be very straightforward in this chapter. So we will be interested in giving a formal definition for the model, and even though the model has a great number of interesting properties, we will focus particularly on the question of criticality. This is the study of a natural parameter of the model, in this case, the probability of having an open edge, and how this model drastically changes depending on the chosen $p$.

As we have stated in the introduction, we are interested in the existence of infinite connected components that contain the origin. We also noted that for $p=1$ we all edges and for $p=0$ we have no edges, so in the first case we almost surely have an infinite connected component and in the other case we almost surely don't have an infinite connected component. So, there exists a non trivial critical point $p_{c}$, such that for $p>p_{c}$ a.s exists an infinite connected component containing the origin and for $p<p_{c}$ we have the opposite? Our first theorems will give a positive answer to this question.

With that in mind we will prove that $p_{c}=1 / 2$. This will use three main tools, which are Harris (FKG) Inequality, proved in the Appendix, the Russo-Seymour-Welsh Theorem, which will give us $p_{c} \geq 1 / 2$, and finally the Margulis-Russo formula, which will prove that $p_{c} \leq 1 / 2$. This chapter is largely based in [9] and [17].

\section{1}

\section{Definition of the model and other basic properties.}

We begin with the graph $\mathbb{Z}^{2}$, which is defined by having its vertices as the points of $\mathbb{Z}^{2}$ and edges between points with Euclidean distance 1. We call the set of edges $E$, and for each $p \in[0,1]$ we define the following random subgraph: Independently for each edge $e \in E$, we keep the edge with probability $p$ and take it away with probability $1-p$. This can also be seen 
as a random configuration $\omega \in \Omega:=\{-1,1\}^{E}$, where for each edge $e \in E$, we independently declare the edge to be open, $w(e)=1$, with probability $p$ or closed, $w(e)=-1$, with probability $1-p$. We remark that depending on the visualization that we want to achieve, we may work with open edges being black lines and closed edges being white (empty) or dashed lines, or we may also work with open edges being red lines and closed edges being blue.

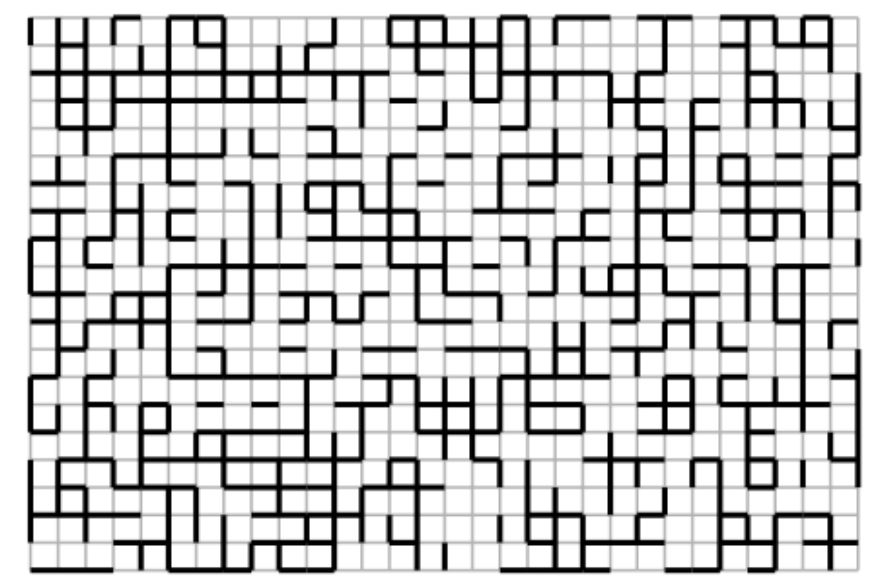

Figure 3.1: Percolation configuration on $\mathbb{Z}^{2}$ for $p=1 / 2$. Image from [9].

We may define a similar model for the triangular lattice $\mathbb{T}$. On this lattice we consider site percolation, which means we are coloring the site as black (red) with probability $p$ and white (blue) with probability $1-p$. Those sites are the points $\mathbb{Z}+e^{i \pi / 3} \mathbb{Z}$ which basically gives us honeycombs. We will only work with $\mathbb{Z}^{2}$ on this chapter, but keep in mind that both this models share many properties.

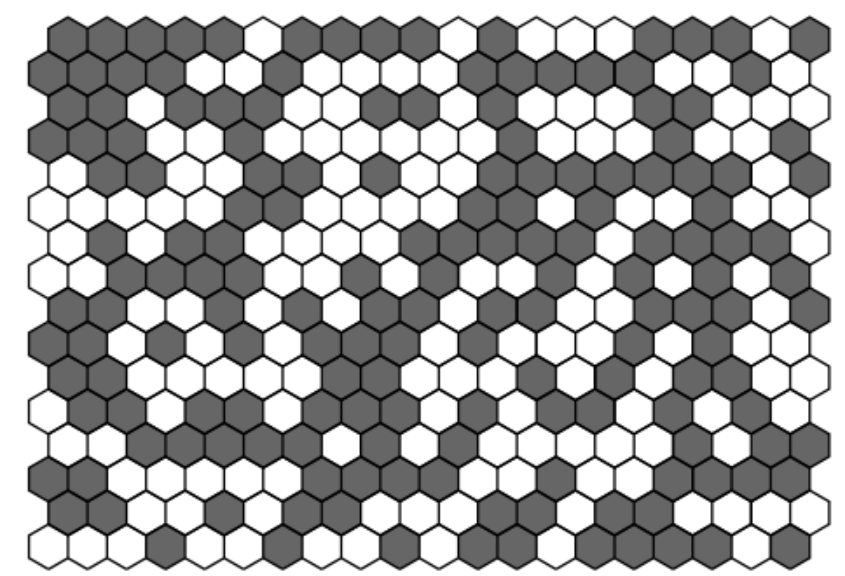

Figure 3.2: Percolation configuration on $\mathbb{T}$ for $p=1 / 2$. Image from [9].

Remark 3.1 We state this a remark because it is not essential to be this rigorous in our context, but for the ones interested in a measure theoretic 
perspective, we define the probability space $\left(\Omega, \mathcal{F}, \mathbb{P}_{p}\right)$, with $\mathcal{F}$ being the $\sigma$ algebra generated by the cylinder sets (events which depend solely on a finite number of edges) and let $\mathbb{P}_{p}=\prod_{e \in E} \mu_{p}(e)$, where $\mu_{p}(1)=p$ and $\mu_{p}(-1)=1-p$.

We let $C(x)$ denote the connected component containing $x$ in our random subgraph. This represents all the vertices connected to $x$ via a path of open edges. We define $C:=C(0)$ and $\theta(p):=\mathbb{P}_{p}(|C|=\infty)$. This represents the probability of having an infinite connected component which contains the origin. Note that as we commented in the Introduction, the existence of a self-avoiding path from 0 to $\infty$ is equivalent to $|C|=\infty$.

We will now prove that for $p$ small but non-zero $\theta(p)=0$ and for $p$ big, but not $1, \theta(p)>0$. This will be the first step towards the understatement of the non trivial critical point which will later turn out to be $1 / 2$.

Theorem 3.2 If $p<1 / 3$, then $\theta(p)=0$

Proof. Consider $F_{n}$ as the event that there is a self-avoiding path of length $n$ starting at 0 using only open edges. Note that

$$
\mathbb{P}_{p}\left(F_{n}\right) \leq 4.3^{n-1} p^{n}
$$

since we have at most $4\left(3^{n-1}\right)$ paths, each with probability $p^{n}$. But note that for $p<1 / 3, \mathbb{P}\left(F_{n}\right) \rightarrow 0$, as $n \rightarrow \infty$. Since we have that $\{|C|=\infty\} \subseteq F_{n}$, for every $n$, then $\theta_{p}=\{|C|=\infty\}=0$.

Theorem 3.3 If $p>2 / 3$, then $\theta(p)>0$.

Before we prove this theorem, we will present a very useful idea that we will be frequently using throughout this thesis.

We introduce the dual graph $\left(\mathbb{Z}^{2}\right)^{*}$ by taking the translation $Z^{2}+\left(\frac{1}{2}, \frac{1}{2}\right)$. Note that there is a $1-1$ correspondence between edges that cross each other at their centers. With this correspondence in mind, we consider an open edge in $\mathbb{Z}^{2}$ to have its correspondent edge as closed in $\left(\mathbb{Z}^{2}\right)^{+}$. So the dual is embedded with a probability measure $\mathbb{P}_{1-p}$.

From this, a key Lemma will allow us to prove the above theorem. Since it is a very geometrical result, we will not give the proof of it but solely state it and refer to Whitney.

Lemma 3.4 $|C|<\infty$ if and only if there exists a simple cycle in $\left(\mathbb{Z}^{2}\right)^{*}$ surrounding 0 consisting of all open edges.

Proof. Pick up $p>2 / 3$. Let $T_{k}$ be the line segment joining the origin to the point $(k, 0)$. Let $C_{2 \ell}$ be a dual open cycle surrounding $T_{k}$, of length $2 l$. Note 


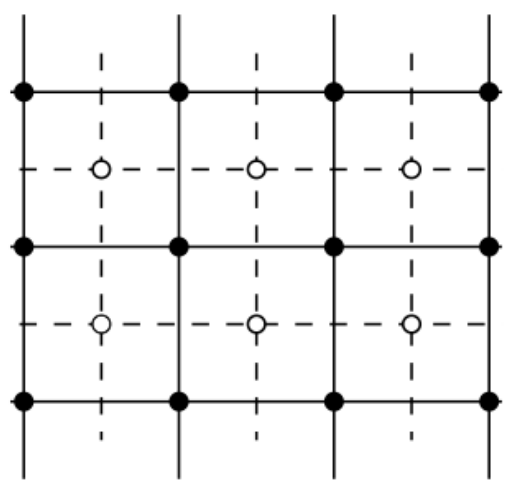

Figure 3.3: This image represents a subgraph of $\mathbb{Z}^{2}$ and its corresponding dual. Note the correspondence between edges that are crossing in the middle. This image was taken from [10].

that $C_{2 \ell}$ must contain a dual open edge $e^{+}$crossing the positive $x$-axis between $k+1 / 2$ and $(2 l-3) / 2$. This means that the number of choices for $e^{*}$ is less than $l$. For the rest of $C_{2 \ell}$ we have a self-avoiding path of length $2 l-1$, so we got at most $4.3^{2 \ell-2}$ choices for it. If we define $Y_{k}$ to be the number of open dual cycles surrounding $T_{k}$ we will get that:

$$
\mathbb{E}_{p}\left(Y_{k}\right) \leq \sum_{\ell \geq k+2} \ell .4 .3^{2 l-2}(1-p)^{2 \ell} \leq \sum_{\ell \geq k+2} \frac{4 \ell}{9}(3(1-p))^{2 \ell}
$$

Since $3(1-p)<1$, the final sum is convergent and we get that $\mathbb{E}_{p}\left(Y_{k}\right) \rightarrow 0$ as $k \rightarrow \infty$. So there exists $k$ such that $\mathbb{E}_{p}\left(Y_{k}\right)<1$. If we define $A_{k}$ to be the event that $Y_{k}=0$ we get that $\mathbb{P}_{p}\left(A_{k}\right)>0$. Define $B_{k}$ to be the event that all the edges of $T_{k}$ are open. Since $A_{k}$ and $B_{k}$ are both independent together with the previous Lemma we get that

$$
\theta(p) \geq P_{p}\left(A_{k} \cap B_{k}\right)>0
$$

So we get the desired result.

Now we may finally define the critical point $p_{c}$ in a natural way by:

$$
p_{c}:=\sup \{p: \theta(p)=0\}=\inf \{p: \theta(p)>0\}
$$

From what was done we already have that $p_{c} \in[1 / 3,2 / 3]$. Now we will present important tools that will help us prove that $p_{c}=1 / 2$. We also note that the reader can have an intuitive idea why this is a natural critical value to think of. A good hint is to prove that the probability of a left to right open cross in a rectangle $[n+1, n]$ should have probability $1 / 2$ by symmetry. A good 
hint is to look at the dual and note that in this case the $\left(\mathbb{Z}^{2}\right)^{*}$ has the same distribution as $\mathbb{Z}^{2}$, so open to bottom open crossings in $\left(\mathbb{Z}^{2}\right)^{*}$ must have the same probabilities as left to right open crossings in $\mathbb{Z}^{2}$. This will be more formalized in the proof of $p_{c} \geq 1 / 2$.

\section{2}

\section{Russo-Seymour-Welsh}

In this section we will state and prove the celebrated result know as the RSW Theorem. First, we will state a more general Lemma valid for all $p \in[0,1]$. It will be the key step to prove the RSW Theorem which is state for $p=1 / 2$. With this Theorem we will easily conclude in the last Section that $p_{c} \geq 1 / 2$. We follow [4] and [17].

First, LR will stand for left-right and TB for top-bottom. Let $H_{m, n}$ be the event that there is a LR open crossing of $[0, m] \times[0, n]$, and $V_{m, n}$ the same but for a TB crossing. If $m=n$ we shorten our notation to $H_{n}$ and $V_{n}$ respectively.

Lemma 3.5 Let $R=[0, m] \times[0,2 n]$, with $m \geq n$ be an $m$ by $2 n$ rectangle. Let $X_{m, 2 n}$ be the event that there two are open paths $\gamma_{1}$ and $\gamma_{2}$, such that $\gamma_{1}$ is a TB cross of the square $S=[0, n] \times[0, n]$, and $\gamma_{2}$ lies within $R$ and joins some vertex on $\gamma_{1}$ to some vertex on the right-hand side of $R$. Then,

$$
\mathbb{P}_{p}\left(X_{m, 2 n}\right) \geq \mathbb{P}_{p}\left(H_{m, 2 n}\right) \mathbb{P}_{p}\left(V_{n}\right) / 2
$$

Proof. Suppose that $V_{n}$ holds. So, there is a path $\gamma_{0}$ of open edges crossing $S$ from top to bottom. We define $\gamma$. the left-most open vertical crossing of $S$ as the open path which is the most to the left. One may note that for every possible TB path $\gamma_{1}$, everything to the right of $\gamma_{1}$ is independent of the event $\left\{\gamma=\gamma_{1}\right\}$, since we only need to examine edges to the left of $\gamma_{1}$ to know if it is the left-most path.

Claim: For any possible TB crossing $\gamma_{1}$, we have that $\mathbb{P}_{p}\left(X_{m, 2 n} \mid \gamma=\right.$ $\left.\gamma_{1}\right) \geq \mathbb{P}_{p}\left(H_{m, 2 n}\right) / 2$.

To prove this, take $\Gamma_{1}$ as the (note necessarily open) path formed by the union of $\gamma_{1}$, with its horizontal reflection $\gamma_{1}^{\prime}$, and one additional edge connecting both of them.

As seen in the image above, this path TB crosses $R$. We also have with probability $\mathbb{P}_{p}\left(H_{m, 2 n}\right)$ the existence of a path $\gamma_{3}$ of open edges LR crossing $R$. Note that this path must meet with $\Gamma_{1}$ in at least one vertex. We also have by symmetry that the probability that some such path meets at a vertex of $\gamma_{1}$ is $\mathbb{P}_{p}\left(H_{m, 2 n}\right) / 2$. So, the event $Y\left(\gamma_{1}\right)$, which is defined as the existence of an open path $\gamma_{2}$ in $R$ to the right of $\Gamma_{1}$, joining a vertex of $\gamma_{1}$ to the right-hand side of 


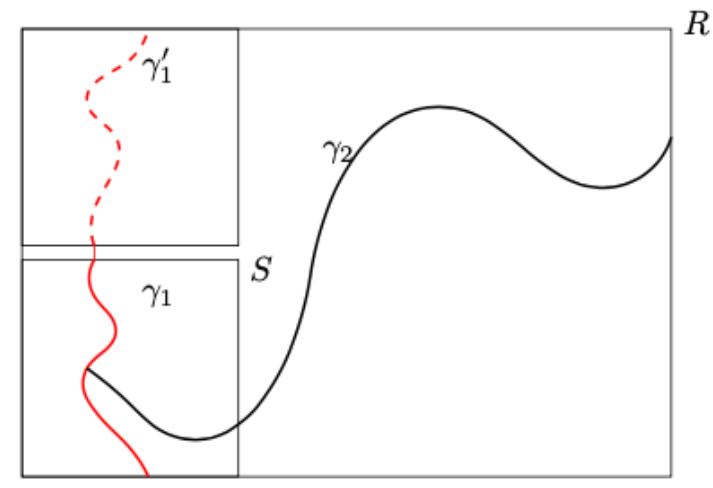

Figure 3.4: Here we represent the rectangle $R$ and the square $S$ along with the curves $\gamma_{2}$ and $\Gamma_{1}$. Observe that the presence of the red and black thick curves implies the event $X_{m, 2 n}$.

$R$, has probability at least $\mathbb{P}_{p}\left(H_{m, 2 n}\right)$. Since this only depends on the edges to the right of $\gamma_{1}$ in $S$, we have by independence that

$$
\mathbb{P}_{p}\left(Y\left(\gamma_{1}\right) \mid \gamma=\gamma_{1}\right)=\mathbb{P}_{p}\left(Y\left(\gamma_{1}\right)\right) \geq \mathbb{P}_{p}\left(H_{m, 2 n}\right) / 2
$$

But the events of $Y\left(\gamma_{1}\right)$ and $\left\{\gamma=\gamma_{1}\right\}$ implies $X_{m, 2 n}$ as highlighted in the drawing. So

$$
\mathbb{P}_{p}\left(X_{m, 2 n} \mid \gamma=\gamma_{1}\right) \geq \mathbb{P}_{p}\left(H_{m, 2 n}\right) / 2
$$

Since $V_{n}$ is the disjoint unions (over all possible curves) of events of the form $\gamma=\gamma_{1}$, we have that $\mathbb{P}_{p}\left(X_{m, 2 n} \mid V_{n}\right) \geq \mathbb{P}_{p}\left(H_{m, 2 n}\right) / 2$. Now we use the conditional probability formula and the fact that $\mathbb{P}_{p}\left(X_{m, 2 n} \cap V_{n}\right)=\mathbb{P}_{p}\left(X_{m, 2 n}\right)$ to conclude that $\mathbb{P}_{p}\left(X_{m, 2 n}\right) \geq \mathbb{P}_{p}\left(H_{m, 2 n}\right) \mathbb{P}_{p}\left(V_{n}\right) / 2$, as desired.

Now, we will go back to formalize the intuition that we gave in the end of the last section.

Lemma 3.6 $\mathbb{P}_{1 / 2}\left(H_{n+1, n}\right)=1 / 2$.

Proof. As we have hinted, we will use the dual lattice to prove it by a symmetry argument. Denote by $B$ the event that there is an open TB crossing of $[1 / 2, n+1 / 2] \times[-1 / 2, n+1 / 2]$ in the dual lattice. We have that by a consequence of Whitney's theorem that $H_{n+1, n}$ occurs if and only if $B$ fails (look at Figure 3.1 again). So, $\mathbb{P}_{1 / 2}\left(H_{n+1, n}\right)+P_{1 / 2}(B)=1$ and by the symmetry that occurs when $p=1 / 2$, we may concluded that $\mathbb{P}_{1 / 2}\left(H_{n+1, n}\right)$. Note that this easily gives us that $\mathbb{P}_{1 / 2}\left(H_{n}\right) \geq 1 / 2$.

Now as a consequence of both these previous lemmas we may prove the following:

Lemma 3.7 For all $n \geq 1$ we have that $\mathbb{P}_{1 / 2}\left(H_{3 n, 2 n}\right) \geq 2^{-7}$. 
Proof. Consider the following subdivision of a $3 n$ by $2 n$ rectangle $R_{0}$ :

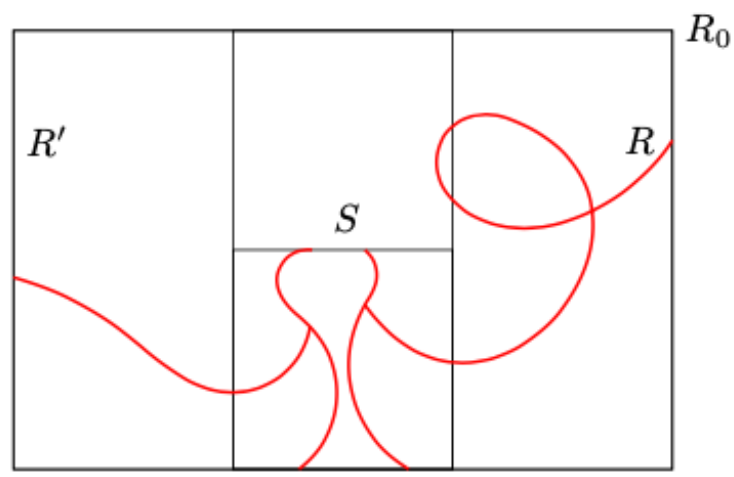

Figure 3.5: Here we divide the original rectangle into three identical $n$ by $2 n$ rectangles. We call $R$ to be the $2 n$ by $2 n$ square formed by the first and second rectangles, and $R^{\prime}$ to be the $2 n$ by $2 n$ square formed by the second and third rectangles. The middle rectangle is subdivided into two identical $n$ by $n$ squares and the lowest one is labeled $S$.

We consider $X_{2 n}^{\prime}$ to be defined analogously as $X_{2 n}$ but reflected horizontally . By Lemma 3.5 we get that

$$
\mathbb{P}_{1 / 2}\left(X_{2 n}^{\prime}\right)=\mathbb{P}_{p}\left(X_{2 n}\right) \geq \mathbb{P}_{1 / 2}\left(H_{2 n}\right) \mathbb{P}_{1 / 2}\left(V_{n}\right) / 2
$$

Note that if the events $X_{2 n}(R), X_{2 n}^{\prime}\left(R^{\prime}\right)$ and $H_{n}(S)$ hold, we have a $3 n$ by $2 n$ crossing of the rectangle $R_{0}$. We changed a little bit the notation to highlight where the crossings are happening. They are also are increasing events, and hence by Harris's Lemma (Theorem A.10):

$$
\begin{aligned}
\mathbb{P}_{1 / 2}\left(H_{3 n, 2 n}\right) & \geq \mathbb{P}_{1 / 2}\left(X_{2 n} \cap X_{2 n}^{\prime} \cap H_{n}\right) \\
& \geq \mathbb{P}_{1 / 2}\left(X_{2 n}\right) \mathbb{P}_{1 / 2}\left(X_{2 n}^{\prime}\right) \mathbb{P}_{1 / 2}\left(H_{n}\right) \\
& \geq \mathbb{P}_{1 / 2}\left(H_{n}\right)^{2} \mathbb{P}_{1 / 2}\left(V_{n}\right)^{2} \mathbb{P}_{1 / 2}\left(H_{n}\right) / 4
\end{aligned}
$$

So, we have as a consequence of Lemma 3.6 that

$$
\mathbb{P}_{1 / 2}\left(H_{3 n, 2 n}\right) \geq 2^{-7}
$$

Now we may finally state the $R S W$ Theorem.

Theorem 3.8 For every $k \geq 1$, there exists $c_{k}>0$ such that for all $n \geq 1$

$$
\mathbb{P}_{1 / 2}(k n, n) \geq c_{k}
$$


The most incredible part is that we have actually made the hardest part with the previous Lemmas. Now it is just a consequence of Harris's Inequality. We sketch the proof for the case $k=2$ and invite the reader to generalize.

Note that as a consequence of Harris's Lemma (See the image below) we have that $\mathbb{P}_{1 / 2}\left(H_{4 n, 2 n}\right) \geq \mathbb{P}_{1 / 2}\left(H_{3 n, 2 n}\right)^{2} \mathbb{P}_{1 / 2}\left(V_{n}\right) \geq 2^{-7} .2^{-7} .2^{-1}=2^{-15}$, for all $n \geq 1$. Small cases such as the rectangle 2 by 1 are trivially done just taking everyone to be open.

With that we conclude this first part. With this powerful tool it will actually be very easy to prove that $p_{c} \geq 1 / 2$. We invite the reader to try to prove this.

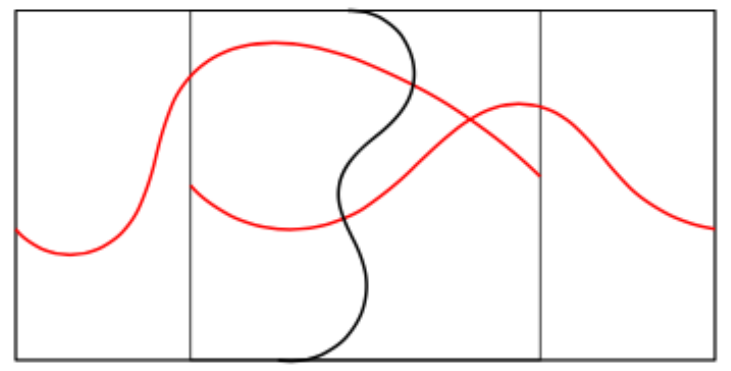

Figure 3.6: We can achieve a crossing in the $4 n$ by $2 n$ rectangle, with two horizontal crossings in $3 n$ by $2 n$ rectangles and a vertical crossing in the $2 n$ by $2 n$ square.

\section{3}

\section{Margulis-Russo Formula}

In this Section we will prove an extremely important result called the Margulis-Russo Formula. This will help us to prove that $p_{c} \leq 1 / 2$. But before we do this we will formally introduce the concept of influence. We have already hinted it in Section 2 and we will give more examples regarding it in Section 5 since this is an extremely important concept for this thesis. First we define the idea of pivotal.

Definição 3.9 Given a Boolean function from $\Omega_{n}$ into $\{-1,1\}$ or $\{0,1\}$. Take a variable $i \in\{1, \ldots, n\}=[n]$. We say that $i$ is pivotal for $f$ for $\omega$ if $f(\omega) \neq f\left(\omega^{i}\right)$, where $\omega^{i}$ denotes $\omega$ but flipped in the ith coordinate. The event $\left\{f(\omega) \neq f\left(\omega^{i}\right)\right\}$ is measurable with respect to $\left\{x_{j}\right\}_{j \neq i}$.

Definição 3.10 The pivotal set $\mathcal{P}$ for $f$, as the random set of $[n]$

$$
\mathcal{P}(\omega)=\mathcal{P}_{f}(\omega):=\{i \in[n]: i \text { is pivotal for } f \text { for } \omega\}
$$


Definição 3.11 (Influence) The influence of the ith bit, $I_{i}(f)$, is defined by:

$$
I_{i}^{p}(f):=\mathbb{P}_{p}(i \text { is pivotal for } f)=\mathbb{P}_{p}\left(f(\omega) \neq f\left(\omega^{i}\right)\right)=\mathbb{P}_{p}(i \in \mathcal{P}) .
$$

We define the total influence $I(f):=\sum_{i=1}^{n} I_{i}(f)$. We also denote by $I I(f):=$ $\sum_{i=1}^{n} I_{i}(f)^{2}$.

For an event $A \in \Omega_{n}$ we denote by $I_{i}(A):=I_{i}\left(\mathbb{1}_{A}\right)$.

We may finally state the Margulis-Russo Formula which connects the derivative of $\mathbb{P}_{p}(A)$ with the influences.

Theorem 3.12 (Margulis-Russo Formula) Let $A \in \Omega_{n}$ be an increasing event. Then,

$$
\frac{d}{d p}=\sum_{i=1}^{n} I_{i}^{p}(A)
$$

Proof. Allow each variable $\omega_{i}$ to have its own parameter $p_{i}$ and let $\mathbb{P}_{p_{1}, \ldots, p_{n}}$ be the corresponding probability measure. It suffices to prove that for $1 \leq i \leq n$

$$
\frac{\partial}{\partial p_{i}} \mathbb{P}_{p_{1}, \ldots, p_{n}}(A)=I_{i}^{p_{1}, \ldots, p_{n}}(A)
$$

Without loss of generality take $i=n$. Given a point $x=\left(x_{1}, \ldots, x_{k}\right) \in \Omega_{k}$, for $k \leq n$, we denote by

$$
p^{x}=\prod_{i: x_{i}=1} p_{i} \prod_{i: x_{i}=-1}\left(1-p_{i}\right)
$$

Note that when $k=n$ we have that $\mathbb{P}_{p}(\{x\})=p^{x}$. For $x \in \Omega_{n-1}$, denote by $x_{+}=\left(x_{1}, \ldots, x_{n-1}, 1\right)$ and $x_{-}=\left(x_{1}, \ldots, x_{n-1},-1\right)$, having $x_{+}, x_{-} \in \Omega_{n}$. We have that $p^{x_{+}}+p^{x_{-}}=p^{x}$.

For an increasing set $A \in \Omega_{n}$, we define

$$
A_{a}=\left\{x \in \Omega_{n-1} \mid x_{+} \in A, x_{-} \in A\right\},
$$

and

$$
A_{b}=\left\{x \in \Omega_{n-1} \mid x_{+} \in A, x_{-} \notin A\right\} .
$$

Since $A$ is increasing we have that

$$
\begin{aligned}
\mathbb{P}_{p_{1}, \ldots, p_{n}}(A) & =\sum_{x \in A_{a}}\left(p^{x_{+}}+p^{x_{-}}\right)+\sum_{x \in A_{b}} p^{x_{+}} \\
& =\sum_{x \in A_{a}} p^{x}+p_{n} \sum_{x \in A_{b}} p^{x}
\end{aligned}
$$


So, we may conclude that

$$
\frac{\partial}{\partial p_{i}} \mathbb{P}_{p_{1}, \ldots, p_{n}}(A)=\sum_{x \in A_{b}} p^{x}
$$

And note that the $x \in A_{b}$ if and only if the nth coordinate is pivotal. So we may conclude the statement

$$
\frac{d}{d p} P_{p}(A)=\sum_{i=1}^{n} I_{i}^{p}(A)
$$

With that we conclude this Section.

\section{4}

The critical point $p_{c}=1 / 2$

Now that we have proved our mains tools, we can finally achieve the desired objective to prove that $p_{c}=1 / 2$. We shall begin proving that $p_{c} \geq 1 / 2$. Theorem $3.13 p_{c} \geq 1 / 2$

Proof. To prove that, we will show that $\theta(1 / 2)=0$, which is, that the probability of having an infinite connected component containing the origin is 0 when $p=1 / 2$. We define a very important concept that will be useful in a number of applications.

Definição 3.14 (Annulus) Let $O(l)$ be the event that there exists an open circuit containing 0 in what we define to be the annulus:

$$
A(l):=[-3 l, 3 l]^{2} \backslash[-l, l]^{2} .
$$

Note that, since the event $O(l)$ happens if we have 4 horizontal crossings in the rectangles $6 l$ by $2 l$ (see the image in the next page), we have by the FKG inequality and Theorem 3.8 that there exists $c>0$ such that $\mathbb{P}_{1 / 2}\left(O_{l}\right) \geq c$ for every $l \geq 1$.

Let $C_{k}$ be the event that there is a circuit in $A\left(4^{k}\right)+1 / 2$ in the dual lattice around the origin consisting of open edges. Since those annulus are disjoint (Try to make a drawing!) we have that the events $C_{k}$ are independent. From what was state before we also have that $\mathbb{P}_{1 / 2}\left(C_{k}\right) \geq c$ for all $k$. If we denote by $E_{k}$ the event that there exists a path connecting the origin to a point outside the area delimited by the last dual annulus $A^{*}(k)$, we have that

$$
\mathbb{P}_{1 / 2}\left(E_{k}\right) \leq \mathbb{P}_{1 / 2}\left(\bigcap_{j=1}^{k} C_{k}^{c}\right)=\prod_{j=1}^{k} \mathbb{P}_{1 / 2}\left(C_{k}^{c}\right) \leq(1-c)^{k},
$$




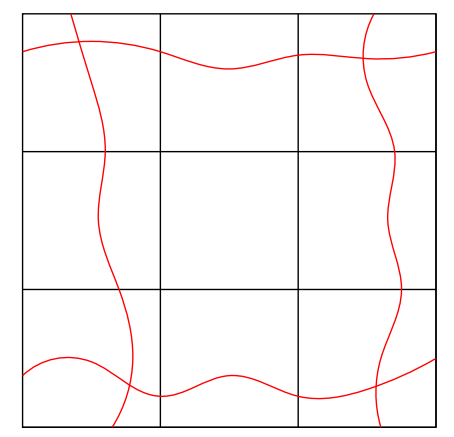

Figure 3.7: Note that the existence of 4 horizontal crossings (hard direction) implies the event $O(l)$. It is then, easy to deduce our claim with the FKG inequality.

for every $k \geq 1$. But we also have that

$$
\theta(1 / 2)=\mathbb{P}_{1 / 2}(|C|=\infty) \leq \mathbb{P}_{1 / 2}\left(\bigcap_{j=1}^{\infty} E_{j}\right)=0
$$

and so we have concluded our proof.

Now, as previously said, we will prove that $p_{c} \leq 1 / 2$, and hence, combine with the previous statement to get $p_{c}=1 / 2$. But this will be a little less straightforward, so we will state and prove a few more helpful lemmas before we do that.

Proposition 3.15 (Finite size criterion) For any $p$, if there exists an $n$ such that

$$
\mathbb{P}_{p}\left(H_{2 n, n-2}\right) \geq 0.98
$$

then $\theta(p)=\mathbb{P}_{p}(|C|=\infty)>0$.

Proof. To prove this, we will first state and prove the following lemma:

Lemma 3.16 For any $\varepsilon \leq .02$, if $\mathbb{P}_{p}\left(H_{2 n, n-2}\right) \geq 1-\varepsilon$, then $\mathbb{P}_{p}\left(H_{4 n, 2 n-2}\right) \geq$ $1-\varepsilon / 2$.

Proof. Let $A_{n}$ be the event that there exists a LR open crossing of $[0,2 n] \times[1, n-$ $1], B_{n}$ the same for $[n, 3 n] \times[1, n-1]$, and $C_{n}$ be the same for $[2 n, 4 n] \times[1, n-1]$. Also, let $D_{n}$ be the event that there exists a TB open crossing of $[n, 2 n] \times[0, n]$, and $E_{n}$ be the same for $[2 n, 3 n] \times[0, n]$.

Note that $\mathbb{P}_{p}\left(D_{n}\right)=\mathbb{P}_{p}\left(E_{n}\right) \geq \mathbb{P}_{p}\left(B_{n}\right)=\mathbb{P}_{p}\left(C_{n}\right)=\mathbb{P}_{p}\left(A_{n}\right) \geq 1-\varepsilon$. So, by the FKG Inequality we have that $\mathbb{P}_{p}\left(A_{n} \cap B_{n} \cap C_{n} \cap D_{n} \cap E_{n}\right) \geq(1-\varepsilon)^{5} \geq 1-5 \varepsilon$. We may see that the intersection of this five events implies the event $H_{4 n, n-2}$. Denoting by $H_{4 n, n-2}^{\prime}$ the event that there exists a LR open crossing of $[0,4 n] \times[n+1,2 n-1]$, we have that $H_{4 n, n-2}$ and $H_{4 n, n-2}^{\prime}$ are independent 
and so

$$
\mathbb{P}_{p}\left(H_{4 n, n-2} \cup H_{4 n, n-2}^{\prime}\right)=1-\left(1-\mathbb{P}_{p}\left(H_{4 n, n-2}^{\prime}\right)\right)^{2} \geq 1-25 \varepsilon^{2} \geq 1-\varepsilon / 2,
$$

since $\varepsilon \leq 0.02$. Since $H_{4 n, n-2} \cup H_{4 n, n-2}^{\prime}$ implies $H_{4 n, 2 n-2}^{\prime}$, we conclude the lemma.

Going back to the proof of the proposition, we pick up $N$ such that $\mathbb{P}_{p}\left(H_{2 N, N-2}\right)$. We have by the previous lemma and a simple inductive argument that for all $k \geq 0$ :

$$
\mathbb{P}_{p}\left(H_{2^{k+1} N, 2^{k} N-2}\right) \geq 1-\frac{.02}{2^{k}}
$$

and so

$$
\sum_{k \geq 0} \mathbb{P}_{p}\left(\left(H_{2^{k+1} N, 2^{k} N}\right)^{c}\right)<\infty .
$$

We will define a sequence $H_{k}$ of crossings of the form $H_{2^{k+1} N, 2^{k} N-2}$ done in a smart configuration. Let $H_{0}$ be a LR open crossing of $[0,2 N] \times[1, N-1], H_{1}$ a TB open crossing of $[1, N-1] \times[0,4 N]$, and so on. See the image below for more details. So, we have by the first Borel-Cantelli Lemma that all but finitely many $H_{k}^{\prime} s$ occur. With that, we may conclude that $\theta_{p}>0$ as desired.

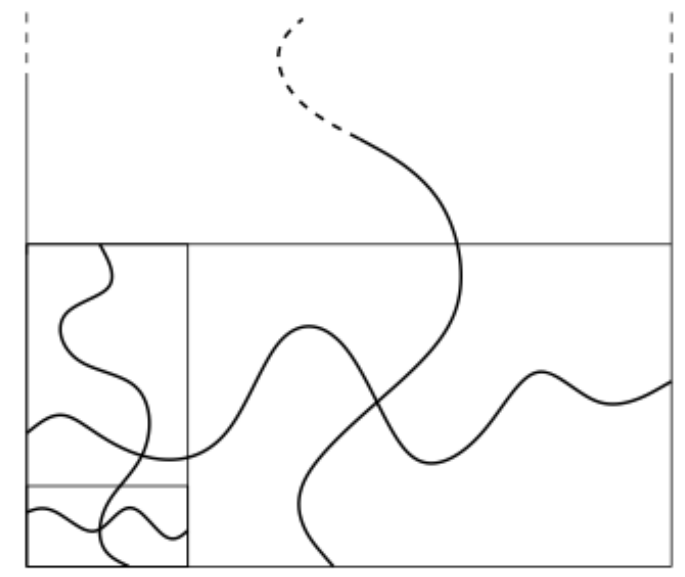

Figure 3.8: Here we denote $H_{0}, H_{1}, H_{2}, H_{3}$, and $H_{4}$. It easy to see that if we consider $\cup_{n \geq k} H_{n}$ it will form an infinite connected component. From there just make a path from the origin to $H_{k}$, which has positive probability for fixed $k$. This image was taken from [9].

Now we will state the following proposition, from which we will easily derive that $p_{c}=1 / 2$. 
Theorem 3.17 Let $\mathcal{P}_{n}$ denote the pivotal set with respect to $\mathbb{1}_{H_{4 n, n}}$. If $p_{c}=$ $1 / 2+\delta_{0}$, with $\delta_{0}>0$, then

$$
\lim _{n \rightarrow \infty} \inf _{p \in\left[1 / 2,1 / 2+\delta_{0}\right]} \mathbb{E}_{p}\left[\left|\mathcal{P}_{n}\right|\right]=\infty
$$

Proof. Since $H_{4 n, n-4 / 2}$ is an increasing event, the RSW Theorem (and by A.9), implies that

$$
\inf _{p \in\left[1 / 2,1 / 2+\delta_{0}\right], n} \mathbb{P}_{p}\left(H_{4 n, n-4 / 2}\right):=\varepsilon_{1}>0 .
$$

Now, define $U_{n}$ to be the event that there is a TB open dual crossing of $[2 n+1 / 2,4 n-1 / 2] \times\left[-1 / 2, n+1 / 2\right.$. We have that there exists $\varepsilon_{2}>0$ such that

$$
\inf _{p \in\left[1 / 2,1 / 2+\delta_{0}\right], n} \mathbb{P}_{p}\left(U_{n}\right):=\varepsilon_{2}>0 .
$$

This is a consequence of the fact that this probability is minimized when $p=1 / 2+\delta_{0} / 2$. So, since $1 / 2+\delta_{0} / 2<p_{c}$, the previous proposition implies that $\mathbb{P}_{1 / 2+\delta_{0} / 2}\left(H_{2 n, n-2}\right) \leq .98$. Since this event and $U_{n}$ are complementary events the claim follows.

Now, if $U_{n}$ occurs, define $\sigma$ to be the right-most open TB crossing. Since $\sigma$ is the right-most crossing, we don't know what happens to the left of $\sigma$. So, conditioned on $\sigma$, by independence, the probability of an open from the left hand side of $[0,4 n] \times[0, n / 2]$ to one step to the left of $\sigma$, has probability at least $\varepsilon_{1}$. Observe that if this happens, the edge one step away from this path is pivotal for $H_{4 n, n}$. If we change its state from, currently closed, to open, there will be a LR open crossing in $[0,4 n] \times[0, n]$. Define $\gamma$ to be the lowest such path if one exists. Again, we know nothing above $\gamma$, so conditioned on both $\gamma$ and $\sigma$, we know nothing about the area delimited at the top-left of these curves. Consider $q$ to be the intersection point of $\sigma$ and $\gamma$. We also consider for each n, the annulus $A\left(4^{k}\right)+1 / 2+q$, which is the previous annulus centered on $q$ instead of 0 , for $k$ such that $4^{k} \leq n / 2$. Again, since $O(l)$ are increasing, by RSW (and A.9), we have that there exists $\varepsilon_{3}>0$, independent of $n, p \in\left[1 / 2,1 / 2+\delta_{0} / 2\right]$, and $k$ such that, with probability at least $\varepsilon_{3}$, there is an open path from $\gamma$ to 1 step to the left of $\sigma$ running within the annulus in the top-left area. Note that each different $k$ gives us a different pivotal edge for $H_{4 n, n}$. Since the number of $k$ 's satisfying $4^{k} \leq n / 2$ goes to infinity with $n$, then

$$
\lim _{n \rightarrow \infty} \inf _{p \in\left[1 / 2,1 / 2+\delta_{0}\right]} \mathbb{E}_{p}\left[\left|\mathcal{P}_{n}\right|\right]=\infty
$$


With that we may finally conclude our main objective!

Theorem $3.18 p_{c} \leq 1 / 2$.

Proof. Suppose that $p_{c}=1 / 2+\delta_{0}$ for a $\delta_{0}>0$. Note that $\mathbb{E}_{p}\left[\left|\mathcal{P}_{n}\right|\right]=I^{p}\left(H_{4 n, n}\right)$. So, by the Margulis-Russo formula we actually have that

$$
\lim _{n \rightarrow \infty} \inf _{p \in\left[1 / 2,1 / 2+\delta_{0}\right]} \frac{d}{d p} \mathbb{P}_{p}\left(H_{4 n, n}\right)=\infty
$$

Since $\delta_{0}>0$, we would have that $\mathbb{P}_{p}\left(H_{4 n, n}\right)=\infty$, for a $p \in\left[1 / 2,1 / 2+\delta_{0}\right]$ by the Mean Value Theorem. This is clearly a contradiction, since probabilities are bounded by 1 . So we conclude that $p_{c} \leq 1 / 2$.

With that, we have proved that $p_{c}=1 / 2$ for Percolation on $\mathbb{Z}^{2}$ and with that, we conclude this Section. 


\section{4}

\section{Voronoi Percolation on $\mathbb{R}^{2}$}

In this Chapter, we will be largely based on the article "Crossing probabilities for Voronoi percolation" by Vincent Tassion [18]. The first Section will be just a simple definition of the model for the readers that are not familiar with Voronoi Percolation. We will also name it annealed Voronoi Percolation, in contrast with the Quenched one.

In the second Section we will prove a RSW version for Voronoi Percolation. That is, if we take an $[k n, n]$ rectangle of $\mathbb{R}^{2}$, and the probability of having an open tile is $1 / 2$, then,

$$
c<\mathbb{P}\left(H_{k n, n}\right)<1-c
$$

, where $c>0$ only depends on $n$.

This is an extremely important result that will be essential for our applications in Chapters 6 and 7. We also mention that the proof generalizes beyond the Voronoi case, requiring only the property of Quasi-Independence. However, since it won't be relevant for our application, we refer the original paper for more details.

It was also noted by Professor Augusto Teixeira that Laurin KoehlerSchindler and Vincent Tassion achieved a prove that does not require the quasiindependence property, only using the FKG inequalities that, for instance, we have in this context. This was not yet published but it can be seen in a seminar uploaded at Youtube named "Russo-Seymour-Welsh theory for FKG percolation" .

This was already conjectured by Tassion, since he already achieved in his paper, without using quasi-independence, the result due to Bollobas and Riordan, in the paper "The critical probability for random Voronoi percolation in the plane is $1 / 2$ " [3] that essentially states that we have a RSW type theorem for an infinite number of rectangles with a fixed aspect ratio. Note that this is indeed weaker than the RSW type result that will be done in this Chapter. We also note that if we have such result we may prove that $p_{c} \geq 1 / 2$ for Voronoi Percolation. One may note that the structure of the lattice doesn't interfere the proof done in Chapter A. Originally, the proof of $p_{c} \geq 1 / 2$ was done with this 
weaker version of the RSW Theorem that can be also found in [4]. But since we already have a stronger result, we won't be interested in such property.

\section{1}

\section{Definition of the model}

We begin with the definition of a Poisson Point Process in $\mathbb{R}^{2}$.

Definição 4.1 For $\lambda>0$, let $\operatorname{Po}(\lambda)$ be a Poisson Point Process in $\mathbb{R}^{2}$ with intensity $\lambda$. That is, the random countably infinite set of points in the plane $\eta$, such that for every bounded Borel set $A$, the probability that $\eta \cap A$ contains exactly $k$ points is

$$
\mathbb{P}_{P o(\lambda)}(|\eta \cap A|=k)=\frac{(\operatorname{vol}(A) \cdot \lambda)^{k}}{k !} e^{-\operatorname{vol}(A) \lambda},
$$

and the random variables $\left|\eta \cap A_{1}\right|, \ldots,\left|\eta \cap A_{n}\right|$ are independent whenever $A_{1}, \ldots, A_{n}$ are disjoint Borel sets.

Definição 4.2 Let $P o(\lambda)$ be as above. For every $u \in \eta$, we define the Voronoi cell of $u$ to be

$$
V(u):=\left\{x \in \mathbb{R}^{2}:\|u-x\|_{2} \leq\|v-x\|_{2} \text { for every } v \in \eta\right\}
$$

Definição 4.3 Given a parameter $p \in[0,1]$, we define the Voronoi Percolation Process, such that for a Poisson Point Process Po $(\lambda)$, we declare each point $z \in \eta$, and its corresponding cell, to be open (black/red) with probability $p$ and closed (white/blue) with probability $1-p$. We may also equivalently define $\eta_{b}$ and $\eta_{w}$ as two independent Poisson process with intensity $\lambda . p$ and $\lambda(1-p)$, and then $\eta=\eta_{b} \cup \eta_{w}$. Points in the boundary of two tiles with different colors are both black and white.

One may note, that again, the probability of a LR open crossing is equal to the probability of a TB closed crossing. Since, apart from a zero measure event, this events are disjoint, we have by duality that

$$
\mathbb{P}\left(H_{n}\right)=1 / 2,
$$

where a $H_{n}$, again, refers to a LR crossing in the $n$ by $n$ square.

The reader may note that there is a spatial dependency in this model so we can't use the same strategy as in $\mathbb{Z}^{2}$. For example, points outside the 
rectangle may affect the tiling inside of it, so the "lowest path" argument won't work as before. But, we have a Quasi-Independence property. That is, we define the annulus $A_{s, t}$ to be

$$
A_{t, s}:=[-t, t]^{2} \backslash[-s, s]^{2}
$$

$O_{s, t}$ to be the event that there is a open circuit around this annulus, and we consider the event

$\mathcal{F}_{s}=\left\{\right.$ for every $z \in A_{4 s, 2 s}$, there exists some point $x \in \eta$ at distance $\left.d(z, x)<s\right\}$, then:

Lemma 4.4 We have that $\lim _{s \rightarrow \infty} \mathbb{P}\left[\mathcal{F}_{s}=1\right]$ and, for any $A_{4 s, 2 s}-$ measurable event $E$, the event $E \cap \mathcal{F}_{s}$ is measurable with respect to the restriction of $\eta_{b}, \eta_{w}$ to $A_{5 s, s}$.

Proof. We consider a covering of $A_{4 s, 2 s}$ given by $C$ Euclidean balls of diameter $s$. Note that this $C$ does not depend on $s$. So the event that each of these balls contains at least one point of $\eta$, implies $\mathcal{F}_{s}$, and so

$$
\mathbb{P}\left[\mathcal{F}_{s}\right] \geq 1-C e^{-\pi s^{2} / 4} .
$$

Observe that for the second part of the Lemma, if $\mathcal{F}_{s}$, no point outside $A_{5 s, s s}$ will interfere in the tiling inside $A_{4 s, s s}$. So, the result follows.

This simple property will be extremely useful, and if the reader notes, it translates the idea that the Voronoi Percolation model has a sort of weak independence, that is, points far from the structures that we are studying won't affect their tilings. With that, we may end this Section.

\section{2}

\section{Russo-Seymour-Welsh for Voronoi Percolation in the plane}

In this Section we will prove the RSW Theorem for annealed Voronoi Percolation. That is:

Theorem 4.5 (RSW for annealed Voronoi Percolation) For every $k \in$ $\mathbb{R}_{+}$, there exists $c_{k} \in(0,1)$ such that for all $s \geq 1$

$$
\mathbb{P}\left(H_{k s, s}\right) \geq c_{k}
$$

Note that by duality we also have

$$
\mathbb{P}\left(H_{k s, s}\right) \leq 1-c_{1 / k} .
$$


While the first result is non trivial for $k>1$, the latter one is non trivial for $k<1$.

Following the same idea of [18], we will state a series of lemmas that will prove that $\inf _{s \geq 1} \mathbb{P}\left(O_{2 s, s}\right)>0$. The following easy corollary of the FKG inequality for annealed Voronoi Percolation with the previous statement will give us the main result. We have proved similar inequalities in Chapter 3, and we recommend the reader to prove this on your own.

Corollary 4.6 As a consequence of the FKG inequality we have that

1. $\mathbb{P}\left(H_{2 s, s}\right) \geq \mathbb{P}\left(O_{2 s, s}\right)$.

2. $\mathbb{P}\left(H_{(1+i \kappa) s, s}\right) \geq \mathbb{P}\left(H_{(1+\kappa) s, s}\right)^{i} \mathbb{P}\left(H_{s}\right)^{i-1}$, for any $\kappa>0$ and $i \geq 1$.

3. $\mathbb{P}\left(O_{2 s, s}\right) \geq \mathbb{P}\left(H_{4 s, s}\right)^{4}$.

But to get the desired statement we will introduce for each scale $s$ a real value $\alpha_{s}$ so we can study the pair $\left(\mathbb{P}\left(O_{2 s, s}, \alpha_{s}\right)\right)$ altogether. First we will prove the RSW result for certain good scales that obey $\alpha_{s} \leq 2 . \alpha_{2 s / 3}$. Then, we will show that those scales are close, in the sense that there exists a global $C$ such that $4 s_{i} \leq s_{i+1} \leq C s_{i}$, where $s_{i}$ satisfies that $\alpha_{s_{i}} \leq 2 \alpha_{2 s_{i} / 3}$. With both this properties we will be able to prove Theorem 4.5.

With that in mind let us introduce the definition of $\alpha_{s}$, which we will call a good scale. But first, we define the following events:

Fix $s \geq 1$. Consider that for $-s / 2 \leq \alpha \leq \beta \leq s / 2$, the event $\mathcal{H}_{s}(\alpha, \beta)$ is defined by the existence of an open crossing in the square $B_{s / 2}:=[-s / 2, s / 2]^{2}$, from the left side to $\{s / 2\} \times[\alpha, \beta]$.

For $0 \leq \alpha \leq s / 2$, we define $\chi_{s}(\alpha)$ to be the event such that:

1. There exists an open path $\gamma_{-1}$ in $B_{s / 2}$ from $\{-s / 2\} \times[-s / 2,-\alpha]$ to $\{-s / 2\} \times[\alpha, s / 2]$.

2. There exists an open path $\gamma_{1}$ in $B_{s / 2}$ from $\{s / 2\} \times[-s / 2,-\alpha]$ to $\{s / 2\} \times[\alpha, s / 2]$.

3. There exists an open path $\gamma^{*}$ in $B_{s / 2}$ from $\gamma_{-1}$ to $\gamma_{1}$.

We also define $\phi_{s}:[0, s / 2] \rightarrow[-1,1]$, a sort of auxiliary function, to be

$$
\phi_{s}(\alpha)=\mathbb{P}\left(\mathcal{H}_{s}(0, \alpha)\right)-\mathbb{P}\left(\mathcal{H}_{s}(\alpha, s / 2)\right)
$$

for $0 \leq \alpha \leq s / 2$. It is not hard to verify that $\phi_{s}$ is continuous, strictly increasing, and $\phi_{s}(0) \leq 0$, by the Voronoi tessellation and $\mathcal{H}_{s}$ properties. We also have by symmetry, that since $H_{s, s}=1 / 2$, this implies that $\phi_{s}(s / 2)=1 / 4$. Now we may finally define $\alpha_{s}$ with the following lemma: 
Lemma 4.7 For every $s \geq 1$, there exists $\alpha_{s} \in[0, s / 4]$ such that the following properties hold:

(P1) For all $0 \leq \alpha \leq \alpha_{s}$, we have that $\mathbb{P}\left(\chi_{s}(\alpha)\right) \geq c_{1}>0$

(P2) If $\alpha_{s}<s / 4$, then for all $\alpha_{s} \leq \alpha \leq s / 2, \mathbb{P}\left(\mathcal{H}_{s}(0, \alpha)\right) \geq 1 / 8+$ $\mathbb{P}\left(\mathcal{H}_{s}(\alpha, s / 2)\right)$.

Proof. Since $\phi_{s}$ is continuous, strictly increasing and we have that $\phi_{s} \leq 0$ and $\phi_{s}(s / 2)=1 / 4$, we may define:

$$
\alpha_{s}:=\min \left(\phi^{-1}(1 / 8), s / 4\right)
$$

Note that with this definition (P2) is clearly satisfied. Now we only need to verify $(\mathbf{P} 1)$. If $\alpha \leq \alpha_{s}$, and since $\mathbb{P}(0, s / 2)=1 / 4$ :

$$
\begin{aligned}
1 & \leq 4 \mathbb{P}\left(\mathcal{H}_{s}(0, s / 2)\right) \\
& \leq 4 \mathbb{P}\left(\mathcal{H}_{s}(0, \alpha)\right)+4 \mathbb{P}\left(\mathcal{H}_{s}(\alpha, s / 2)\right) \\
& \leq 4 \phi_{s}(\alpha)+8 \mathbb{P}\left(\mathcal{H}_{s}(\alpha, s / 2)\right) \\
& \leq 1 / 2+8 \mathbb{P}\left(\mathcal{H}_{s}(\alpha, s / 2)\right)
\end{aligned}
$$

So, for every $\alpha \leq \alpha_{s}$

$$
\mathbb{P}\left(\mathcal{H}_{s}(\alpha, s / 2)\right) \geq 1 / 16
$$

We have that 4 events of the type $H_{s}(\alpha, s / 2)$ with a TB open crossing of $B_{s / 2}$ implies the event $\chi_{s}(\alpha)$ (see the image below for more details). So, by the FKG inequality for annealed Voronoi Percolation ( i.e. doesn't depend on a fixed distribution of points. This difference will be made more clear when we

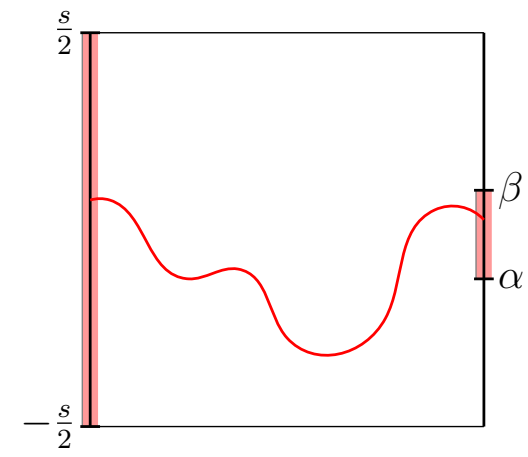

Figure 4.1: We represent the event $\mathcal{H}_{s}(\alpha, \beta)$.

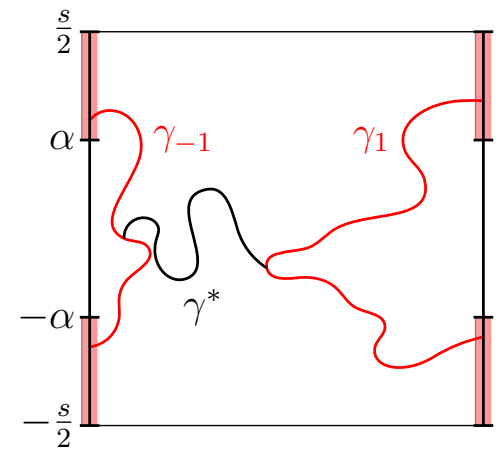

Figure 4.2: We represent the event $\chi_{s}(\alpha)$. 
introduce the notion of Quenched Voronoi Percolation):

$$
\mathbb{P}\left(\chi_{s}(\alpha)\right) \geq \frac{1}{2}\left(\frac{1}{16}\right)^{4}=: c_{1} .
$$

With that we conclude the proof.

We represent below an image that will facilitate the visualization and a more detailed explanation of what is in fact happening above. Since it didn't fit in this page, we leave a blank space and present it in the next page. 


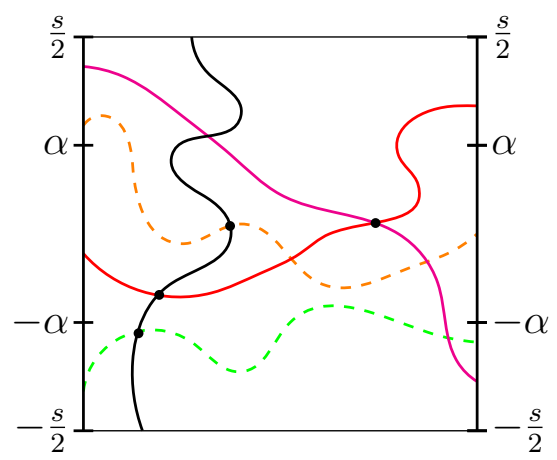

Figure 4.3: The thick lines represent the events $\mathcal{H}_{s}(\alpha, s / 2)$ (red) and $\mathcal{H}_{s}(-s / 2,-\alpha)$ (magenta). The dashed lines represent reflected version $\mathcal{H}_{s}^{*}(\alpha, s / 2)$ (orange) and $\mathcal{H}_{s}^{*}(-s / 2,-\alpha)$. The black curve represents the TB open crossing. Note that we can always form an event of the type $\chi_{s}(\alpha)$. Just pick the dashed lines, if they inintersectiontersect to the left of the black, curve pick this and everything to the left of the intersection point as $\gamma_{-1}$. If not, pick the curve formed by the black curve and the intersection points with the dashed lines and look for the left of it as the image above. For the thick lines, if they intersect to the right of the black curve pick this and everything to the right of the intersection point as $\gamma_{1}$. This is the case of the image above. If not, to the same thing as before but substituting left for right. Finally, the black curve will be $\gamma^{*}$ itself or we will be able to find a path from the intersection point to the black curve, in the image the red path. We also remark, that exceptionally, in this Chapter, we represent open path with multiple colors to aid the visualization. We only avoid the color blue to avoid confusion.

Lemma 4.8 There exists $c_{2}>0$ such that for all $s \geq 2$, the inequality $\alpha_{s} \leq 2 \alpha_{2 s / 3}$ implies

$$
\mathbb{P}\left(O_{2 s, s}\right) \geq c_{2}
$$

Proof. First, consider the case $\alpha_{s}=s / 4$. In this case we won't even need the hypothesis $\alpha_{s} \leq 2 \alpha_{2 s / 3}$. By (P1) we have that $\mathbb{P}\left(\chi_{s}(s / 4)\right) \geq c_{1}$. With that in mind, let's create an open TB crossing in the rectangle $[0, s] \times[0,2 s]$. Just consider for $i=0, \ldots, 3$ the event $\varepsilon_{i}$ that there exists an open path from $\{0\} \times[(i-1) s / 2, i s / 2]$ to $\{0\} \times[(i+1) s / 2,(i+2) s / 2]$ in the strip $[0, s] \times \mathbb{R}$. We have that $\mathbb{P}\left(\varepsilon_{i}\right) \geq \mathbb{P}\left(\chi_{s}(s / 4)\right)$, (note that if we translate by $s / 4$ in the vertical direction $\gamma_{-1}$ implies an event of the type $\varepsilon_{i}$ )and the in the intersection of all this events implies $V_{s, 2 s}$. So, by rotational invariance, $\mathbb{P}\left(H_{2 s, s}\right) \geq c_{1}^{4}$. And hence, by Corollary 4.6

$$
\mathbb{P}\left(O_{2 s, s}\right) \geq\left(c_{1}^{12}(1 / 2)^{2}\right)^{5}
$$




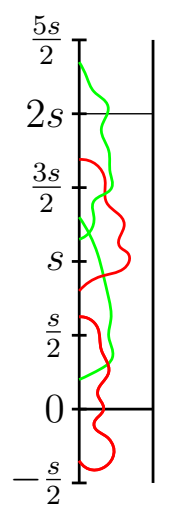

Figure 4.4: Here we represent the events $\varepsilon_{0}, \varepsilon_{1}, \varepsilon_{2}$, and $\varepsilon_{3}$. Note that since they all intersect, we may form a TB open crossing in the rectangle $[0, s] \times[0,2 s]$.

Now, consider $s$ to be such that $\alpha_{s} \leq 2 \alpha_{2 s / 3}$ and $\alpha_{s}<s / 4$. We will use the event $\chi_{2 s / 3}\left(\alpha_{2 s / 3}\right)$ to connect two crossings and wield a horizontal crossing in a rectangle of aspect ratio $4 / 3$. To do so, consider the two squares $R:=\left(-s / 6,-\alpha_{2 s / 3}\right)+B_{s / 2}$ and $R^{\prime}:=\left(s / 6,-\alpha_{2 s / 3}\right)+B_{s / 2}$. Since $\alpha_{2 s / 3} \leq s / 6$ (by definition) we have that $B_{s / 3} \subset R$ and $B_{s / 3} \subset R^{\prime}$. Let $\varepsilon$ be the event defined by the existence of an open path from left to $\{s / 3\} \times\left[-\alpha_{2 s / 3}, \alpha_{2 s / 3}\right]$ in R. Analogously, define $\varepsilon^{\prime}$ as the event that there exists an open path from $\{-s / 3\} \times\left[-\alpha_{2 s / 3}, \alpha_{2 s / 3}\right]$ to right in $R^{\prime}$. Since $\alpha_{s} \leq 2 \alpha_{2 s / 3} \leq s / 2$ and $\alpha_{s}<s / 4$, (P2) gives us that $\mathbb{P}\left(\mathcal{H}_{s}\left(0,2 \alpha_{2 s / 3}\right)\right) \geq 1 / 8$. So, $\mathbb{P}(\varepsilon) \geq 1 / 8$ and $\mathbb{P}\left(\varepsilon^{\prime}\right) \geq 1 / 8$.Also, recall that $\mathbb{P}\left(\chi_{2 s / 4}\left(\alpha_{2 s / 3}\right)\right) \geq c_{1}$ by $(\mathbf{P} 1)$.

See the image in the next page, and observe that if the events $\chi_{2 s / 3}, \varepsilon$ and $\varepsilon^{\prime}$ occur, there must exist a LR open path in $R \cup R^{\prime}$. Since $R \cup R^{\prime}$ has aspect ratio $4 / 3$, the FKG inequalities implies that

$$
H_{4 s / 3, s} \geq \mathbb{P}\left(\chi_{2 s / 3}\left(\alpha_{2 s / 3}\right) \cap \varepsilon \cap \varepsilon^{\prime}\right) \geq c_{1}(1 / 8)^{2} .
$$

Corollary 4.6 ends our proof.

Now we will aim to prove the second part, which is, showing the existence of a global constant $C \geq 4$ such that $4 s_{i} \leq s_{i+1} \leq C s_{i}$, where the sequence $\left\{s_{i}\right\}$ satisfies that $\mathbb{P}\left(O_{2 s_{i}, s_{i}}\right)$. But, before we do it, we will need to prove two preliminary lemmas. 


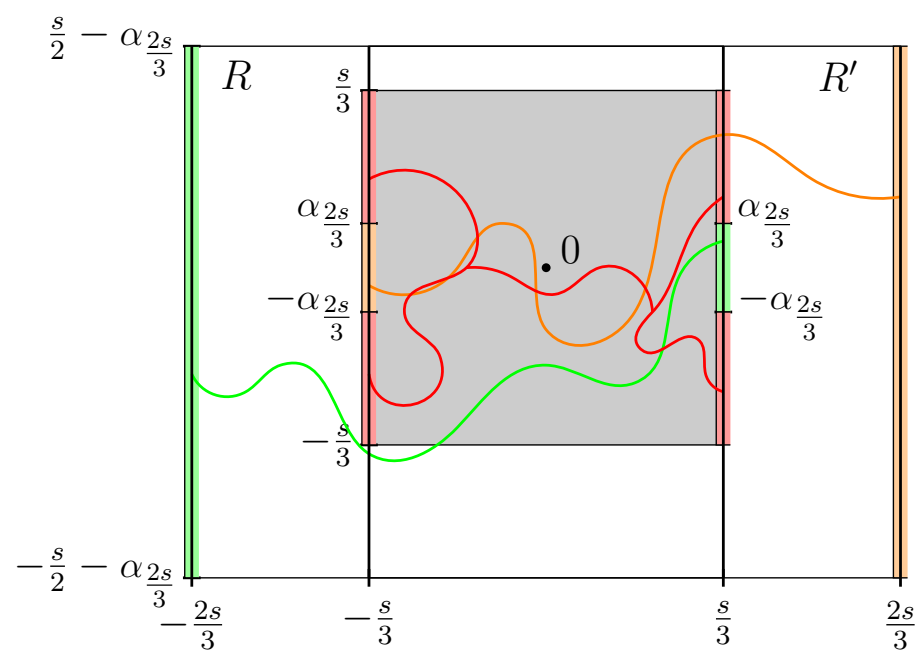

Figure 4.5: The intersection of the events $\chi_{2 s / 3}\left(\alpha_{2 s / 3}\right), \varepsilon$ and $\varepsilon^{\prime}$ implies the existence of a LR crossing in $R \cup R^{\prime}$.

Lemma 4.9 There exists $c_{3}>0$ such that for all $s \geq 1$ and $t \geq 4 s$

$$
\text { If } \mathbb{P}\left(O_{2 s, s}\right) \geq c_{2} \text { and } \alpha_{t}<s \text {, then } P\left(O_{2 t, t}\right) \geq c_{3} \text {. }
$$

Proof. Let $s \geq 1$ and $t \geq 4 s$. Assume that $\mathbb{P}\left(O_{2 s, s}\right) \geq c_{2}$ and $\alpha_{t}<s$. Consider the event $E$ that there exists

1. An open path from left to $\{0\} \times[0, s]$ in the square $[-t, 0] \times[-t / 2, t / 2]$.

2. An open from $\{0\} \times[0, s]$ to right in the square $[0, t] \times[-t / 2, t / 2]$.

3. An open circuit in the annulus $A_{2 s, s}$.

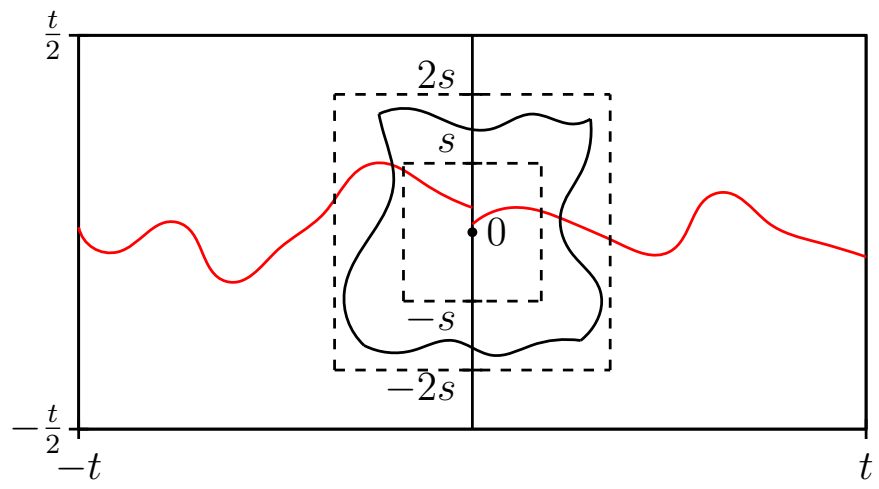

Figure 4.6: The event $E$.

Since $\alpha_{t}<s \leq t / 4$, property (P2) implies that for $\alpha_{t} \leq \alpha \leq t / 2$

$$
\mathbb{P}\left(\mathcal{H}_{t}(0, \alpha)\right) \geq c_{0} / 4
$$


In particular, we may take $\alpha=s$. So, by the FKG inequality

$$
\mathbb{P}\left(H_{2 t, t}\right) \geq\left(\frac{c_{0}}{4}\right)^{2} c_{2}
$$

With that, we have by Corollary 4.6 that

$$
\mathbb{P}\left(O_{2 t, t}\right) \geq c_{3}
$$

for some $c_{3}>0$.

For the next lemma we will need to use Lemma 4.4. So, pick up $s_{0}$ such that for all $s \geq s_{0}$

$$
\mathbb{P}\left(\mathcal{F}_{s}\right) \geq 1-c_{3} / 2
$$

Lemma 4.10 Define the constant $C_{1} \geq 25$, sufficiently big such that

$$
\left(1-\frac{c_{3}}{2}\right)^{\left\lfloor\log _{5}\left(C_{1}\right)\right\rfloor-1}<\frac{c_{0}}{4} .
$$

Let $s \geq s_{0}$ such that $\mathbb{P}\left(O_{2 s, s}\right) \geq c_{2}$. Then, there exists $s^{\prime} \in\left[4 s, C_{1} s\right]$ such that $\alpha_{s^{\prime}} \geq s$.

Proof. Let $s \geq s_{0}$ such that $\mathbb{P}\left(O_{2 s, s}\right) \geq c_{2}$. Suppose by contradiction that $\alpha_{t}<s$, for all $4 s \geq t \geq C_{1} s$. For $t=C_{1} s$, we have that $\alpha_{C_{1} s}<C_{1} s / 4$ and $\alpha_{C_{1} s}<s<C_{1} s / 2$. So by (P2) we have that

$$
\mathbb{P}\left(\mathcal{H}_{C_{1} s}(0, s)\right)-\mathbb{P}\left(\mathcal{H}_{C_{1} s}\left(s, C_{1} s / 2\right)\right) \geq c_{0} / 4
$$

Let $1 \leq i \leq\left\lfloor\log _{5}\left(C_{1}\right)\right\rfloor-1$. Since $\mathbb{P}\left(O_{2 s, s}\right) \geq c_{2}$ and $\alpha_{5^{i} s}<s$, Lemma 4.9 applied to $t=5^{i} s$ gives us $\mathbb{P}\left(O_{2.5^{i} s, 5^{i} s}\right)$. Together with the independence property of Lemma 4.4

$$
\mathbb{P}\left(O_{2.5^{i} s, s} \cap \mathcal{F}_{5^{i} s / 2}\right) \geq c_{3} / 2
$$

Let $\varepsilon$ be the event that there exists an open circuit around $A_{C_{1} / 2, s}$. This happens when $A_{2.5^{i} s, 5^{i} s} \cap \mathcal{F}_{5^{i} s / 2}$ occurs for any $1 \leq i \leq\left\lfloor\log _{5}\left(C_{1}\right)\right\rfloor-1$. By Lemma 4.4 this events are independent and we get:

$$
\begin{aligned}
\mathbb{P}\left(\varepsilon^{c}\right) & \leq \mathbb{P}\left(\bigcap_{1 \leq i \leq\left\lfloor\log _{5}\left(C_{1}\right)\right\rfloor-1}\left(O_{2.5^{i} s, 5^{i} s} \cap \mathcal{F}_{5^{i} s / 2}\right)^{c}\right) \\
& =\prod_{\left.1 \leq i \leq \log _{5}\left(C_{1}\right)\right\rfloor-1} \mathbb{P}\left(\left(O_{2.5^{i} s, 5^{i} s} \cap \mathcal{F}_{5^{i} s / 2}\right)^{c}\right) \\
& \leq\left(1-\frac{c_{3}}{2}\right)^{\left\lfloor\log _{5}\left(C_{1}\right)\right\rfloor-1}<\frac{c_{0}}{4} .
\end{aligned}
$$


Now, consider the event $\Omega$ that in the square $\left[-C_{1} s, 0\right] \times\left[-C_{1} s / 2, C_{1} s / 2\right]$ :

1. There is no open path from the left side to $\{0\} \times\left[s, C_{1} s / 2\right]$.

2. There exists an open path from the left side to $\{0\} \times[0, s]$.

Observe that this implies that there can't be an open circuit around $A_{C_{1} s / 2, s}$.

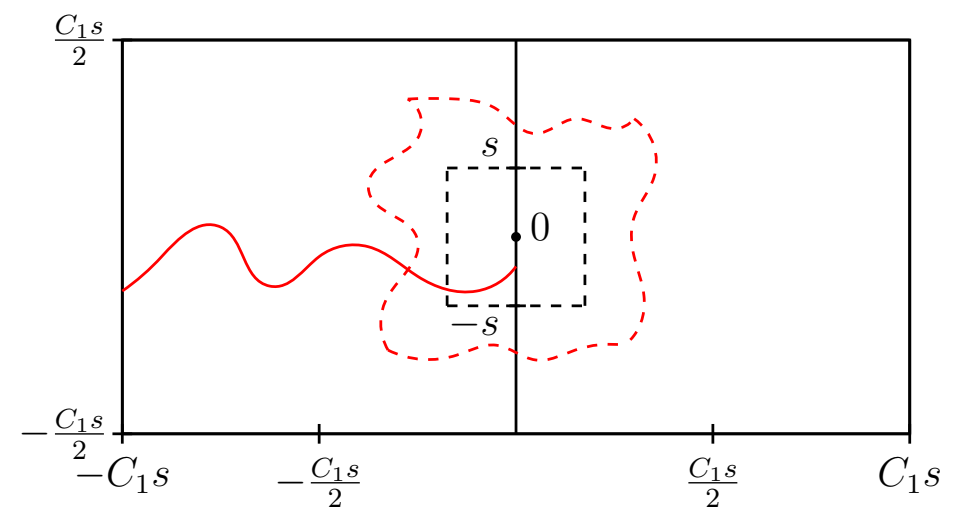

Figure 4.7: The event $\Omega$. The event $\varepsilon$ represented by the dashed lines can't happen, otherwise, $\Omega$ won't wield.

So, we obtain that

$$
\mathbb{P}\left(\mathcal{H}_{C_{1} s}(0, s)\right)-\mathbb{P}\left(\mathcal{H}_{C_{1} s}\left(s, C_{1} s / 2\right)\right) \leq \mathbb{P}(\Omega) \leq \mathbb{P}\left(\varepsilon^{c}\right)<c_{0} / 4
$$

which contradicts the assumption. So, we wield the result.

Lemma 4.11 There exists a constant $C_{3} \geq 25$ and an infinite sequence $s_{1}, s_{2}, \ldots$ of scals such that for all $i \geq 1$,

1. $4 s_{i} \leq s_{i+1} \leq C_{3} s_{i}$.

2. $\mathbb{P}\left(O_{2 s_{i}, s_{i}}\right) \geq c_{2}$.

Proof. Since $\alpha_{s} \leq s$, there must exist $s_{1} \geq s_{0}$ such that $\alpha_{s_{1}} \leq 2 \alpha_{2 s_{1} / 3}$. So, by Lemma 4.8 , we have that $\mathbb{P}\left(O_{2 s_{1}, s_{1}}\right) \geq c_{2}$. So, by Lemma 4.11 there exists $s_{1}^{\prime} \in\left[4 s, C_{1} s\right]$ such that

$$
\alpha_{s_{1}^{\prime}} \geq s_{1} \geq s_{1}^{\prime} / C_{1} .
$$

So, there must exist $s_{2} \in\left[s_{1}^{\prime}, \frac{3}{2} C_{1}^{\log _{4 / 3}(3 / 2)} s_{1}^{\prime}\right]$ such that $\alpha_{s_{2}} \leq 2 \alpha_{2 s_{2} / 3}$ or the bound $\alpha_{s} \leq s$ would be contradicted. Let $C_{3}:=\frac{3}{2} C_{1}^{\log _{4 / 3}(3 / 2)}$. We have that $s_{2} \in\left[4 s_{1}, C_{3} s_{1}\right]$ and by Lemma $4.8, \mathbb{P}\left(O_{2 s_{2}, s_{2}}\right) \geq c_{2}$.

Note that the constant $C_{3}$ is independent, and so, we can iterate the construction above, and find by induction $s_{3}, s_{4}, \ldots$ 
So, we have at each scale $s_{i}$ that $\mathbb{P}\left(H_{2_{i}, s_{i}}\right) \geq c_{2}$. We may pick $5 C_{3}$ rectangles of the form $\left[s_{i} . j,(2+j) s_{i}\right] \times\left[0, s_{i}\right]$, with $0 \leq j \leq 5 C_{3}$, and $5 C_{3}$ squares of the form $\left[s_{i} \cdot j, s_{i}(j+1)\right] \times\left[0, s_{i}\right]$, consider a LR open crossing in the rectangles and a TB open crossing in all the squares. With that we have that $\mathbb{P}\left(H_{2 s, s}\right) \geq c>0$ for all $s \in\left[s_{i}, C_{3} s_{i}\right]$. We may do this for all $s_{i}$ since $4 s_{i} \leq s_{i+1} \leq C_{3} s_{i}$. So, we get that $\mathbb{P}\left(H_{2 s, s}\right) \geq c>0$ for all $s \geq 1$. By Corollary 4.6, we may end this Chapter with the proof that for every $k>0$, there exists $c_{k}$ such that for all $s \geq 1$ :

$$
c_{k} \leq \mathbb{P}\left(H_{k s, s}\right) \leq 1-c_{k} .
$$




\section{Noise Sensitivity of Boolean Functions}

In this Chapter, we present the definition of Noise Sensitivity. It was first developed by Benjamini, Kalai and Schramm in the paper [2]. This concept is not restricted to the Percolation setting, but its most important application in the original paper was showing that for $p=1 / 2$, crossings on $\mathbb{Z}^{2}$ are asymptotically Noise Sensitive events. This means that if for a growing sequence of rectangles, we know all edges, but a small random set, we can 't predict if there will have a LR crossing or not.

In the first Section we will review the notion of influence, applying it to more simple examples that were previously define in Chapter 2. We also use Fourier Analysis on the Hypercube, presented in Chapter 2. This will turn out to be an essential tool for the study of Influence and Noise Sensitivity.

In the second Section we will formally state the definition of asymptotically noise sensitive functions. We will also give a condition on the Fourier spectrum and prove that it is equivalent to noise sensitivity.

In the third Section we will prove show that noise sensitivity is linked to Influences. We prove (with a slightly strengthened hypothesis) the BenjaminiKalai-Schramm Noise Sensitivity Theorem, which we now state.

Theorem 5.1 [2] If

$$
\lim _{n \rightarrow \infty} \sum_{k=1}^{n} I_{k}\left(f_{n}\right)^{2}=0,
$$

then $\left\{f_{n}\right\}$ is asymptotically noise sensitive.

This is an extremely non-trivial result that was used by the authors to prove that crossings on $\mathbb{Z}^{2}$ are noise sensitive at $p_{c}=1 / 2$. We will not reproduce their proof on the noise sensitivity of crossing events. Instead, we shall see how their ideas may be combined with an analysis of randomized algorithms, as was done in [15]. The approach of [15] gives an upper bound for the sum of squares of influences in terms of the revealment $\delta_{\mathcal{A}}(f)$ of a randomized algorithm $\mathcal{A}$ applied to a function $f$. Both these concepts, and the proof of their result will be carefully done in the fourth Section.

Finally, we apply it in the last Section for crossings. We will do it explicitly for the hexagonal lattice $\mathbb{T}$ but it will be pointed out that our proof is actually more general. 
This Chapter will be practically self-contained, the only significant result that we use from Percolation is the Russo-Seymour-Welsh Theorem. It is also largely based on the results of Schramm and Steif [15] and the book of Garban and Steif [9].

\section{1}

\section{Influence}

We begin by quickly reminding the definition of influence.

Definição 5.2 Let $\omega \in \Omega_{n}$, and $f: \Omega_{n} \rightarrow\{-1,1\}$ or $\{0,1\}$. The influence of the ith bit, $I_{i}(f)$, is

$$
I_{i}(f):=\mathbb{P}\left(f(\omega) \neq f\left(\omega^{i}\right)\right)=\mathbb{P}(i \in \mathcal{P})
$$

where $\mathcal{P}$ denotes the pivotal set for $f$.

We will now compute the influence of some of the functions we introduced along this thesis.

Example 5.3 (Dictator) The Dictator function, given by $\operatorname{Dict}_{n}\left(x_{1}, \ldots, x_{n}\right)=$ $x_{1}$ must have have influence only on the 1 th bit, if we expect a natural definition. Note that by our definition:

$$
I_{1}(\operatorname{Dict})=\mathbb{P}\left(\operatorname{Dict}(\omega) \neq \operatorname{Dict}\left(\omega^{1}\right)\right)=1,
$$

because if we flip the value of the first bit, the value of the function will change. For $i \neq 1$

$$
I_{i}(\operatorname{Dict})=\mathbb{P}\left(\operatorname{Dict}(\omega) \neq \operatorname{Dict}\left(\omega^{i}\right)\right)=0,
$$

since the function depends only on the first value. Note that the total influence $I(f)=1$. Thus, we achieve something expected.

Example 5.4 (Example) For the parity function, given by $\operatorname{Par}_{n}\left(x_{1}, \ldots, x_{n}\right)=$ $\prod_{i=1}^{n} x_{i}$, we expected all the influences to have equal and maximal value. Note that by our definition, for any $1 \leq i \leq n$

$$
I_{i}(\operatorname{Par}) \mathbb{P}\left(\operatorname{Par}(\omega) \neq \operatorname{Par}\left(\omega^{i}\right)\right)=1,
$$

since if we flip the value of the ith bit, we change the outcome of the function. Note that in this case, the total influence $I(f)=n$. This extreme case has another interesting property. Even if we know all but one voters, we still won't know the outcome of this functions. This points us towards the next definition we will introduce, which is noise sensitive functions, i.e., functions that gives 
us few information, and are highly likely to change, if we make small random disturbances on it.

Example 5.5 (Majority) Majority function is defined for odd $n$ as $\operatorname{Maj}_{n}\left(x_{1}, \ldots, x_{n}\right)=\operatorname{sign}\left(\sum_{i=1}^{n} x_{i}\right)$. We expected that all the voters would have the same influence, and it would be somehow balanced, opposed to the previous extreme cases. Note that, by our definition

$$
I_{1}(M a j):=\mathbb{P}\left(\operatorname{Maj}(\omega) \neq \operatorname{Maj}\left(\omega^{1}\right)\right) .
$$

Note that for the first bit to change the outcome of the function, the sum of $x_{2}+\ldots+x_{n}$ must equal 0 . This happens when we have $\frac{n-1}{2} 1^{\prime} s$, and $\frac{n-1}{2}-1^{\prime}$ s. Thus, the probability of such event must be given by

$$
I_{1}(M a j)=\frac{\left(\begin{array}{l}
n-1 \\
\frac{n-1}{2}
\end{array}\right)}{2^{n-1}}=\Theta\left(\frac{1}{\sqrt{n}}\right),
$$

by Stirling's Approximation (A.7), where $\Theta(m)$ denotes the same order as $m$. By symmetry, we may easily see that $I_{i}(M a j)=\Theta(1 / \sqrt{n})$, for every $1 \leq i \leq n$. So, the total influence $I(f)=n / \sqrt{n}$.

Example 5.6 (Crossings on $\mathbb{Z}^{2}$ or $\mathbb{T}$ ) Let $f_{n}$ be defined as the indicator function for the existence of a LR open crossing in a grid (hexagonal lattice) of $n$ edges (sites). Edges that sufficiently far from the boundary will be pivotal when the so-called 4-arm event happens. This is more natural in $\mathbb{T}$ since the dual $\mathbb{T}^{*}=\mathbb{T}$. But, such events may be seeing in a similar for $\mathbb{Z}^{2}$ as we have done in Theorem 3.17. It is still extremely hard to predict the decay of such event. It was proved by Smirnov and Werner in [16] that for the hexagonal lattice, if $R>1$ is the radius of the circle centered in site 0 , then

$$
\mathbb{P}\left[A_{R}^{4}\right]:=\alpha_{4}(R)=R^{-\frac{5}{4}+o(1)},
$$

where $A_{R}^{4}$ denotes the event that there are four arms of alternating color from the site 0 to distance $R$ away. This is an extremely technical result and we won't prove it, since it is out of the scope of this dissertation. We may also similarly define the 1-arm event, $A_{R}^{1}$, as the event that the site 0 is connected to distance $R$ by some open path, and $A_{R}^{2}$ be the event that there are two arms of different colors from site 0 to distance $R$ away.

We end this section by relating the notion of influence to that of the energy spectrum defined in the Fourier section. 


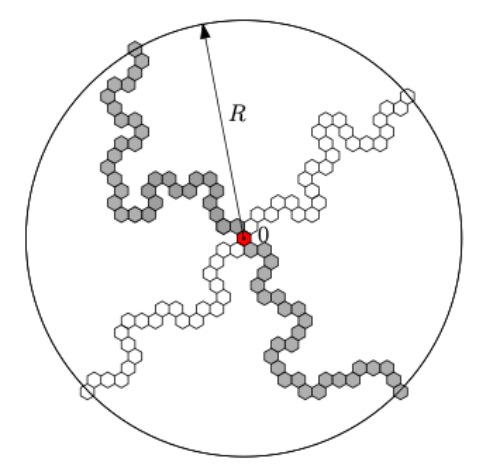

Figure 5.1: A realization of the four-arm event on the hexagonal lattice $\mathbb{T}$. This picture was taken from [9].

Proposition 5.7 If $f: \Omega_{n} \rightarrow\{0,1\}$, then for all $k$,

$$
I_{k}(f)=4 \sum_{S: k \in S} \hat{f}(S)^{2}
$$

and

$$
I(f)=4 \sum_{S}|S| \hat{f}(S)^{2}
$$

Proof. For $f: \Omega_{n} \rightarrow \mathbb{R}$ we introduce a function, corresponding to a discrete derivative along the $k t h$ bit, that will be quite useful for a number of application. Let for all $k \in[n], \nabla_{k}(f): \Omega_{n} \rightarrow \mathbb{R}$ be defined as

$$
\nabla_{k} f(\omega)=f(\omega)-f\left(\sigma_{k}(\omega)\right)
$$

where $\sigma_{k}$ maps $\Omega_{n}$ to itself by flipping the $k t h$ bit. Note that:

$$
\nabla_{k} f(\omega)=\sum_{S \subseteq\{1, \ldots, n\}} \hat{f}(S)\left[\chi_{S}(\omega)-\chi_{S}\left(\sigma_{k}(\omega)\right)\right]=\sum_{S \subseteq\{1, \ldots, n\}, k \in S} 2 \hat{f}(S) \chi_{S}(\omega)
$$

With that, we get that for any $S \subseteq[n]$,

$$
\widehat{\nabla_{k}} f(S)= \begin{cases}2 \hat{f}(S), & \text { if } k \in S \\ 0, & \text { otherwise. }\end{cases}
$$

If $f$ maps into $\{0,1\}, \nabla_{k} f$ maps into $\{-1,0,1\}$, and we have that $I_{k}(f)=\left\|\nabla_{k} f\right\|_{1}$, and $\left\|\nabla_{k} f\right\|_{1}=\left\|\nabla_{k} f\right\|_{2}^{2}$. So, applying Parseval's formula and using the previous characterization of $\widehat{\nabla_{k}} f(S)$ we wield the first statement of the proposition. The second one derives easily from it. 
We remark that if $f: \Omega_{n} \rightarrow\{-1,1\}$, we get that $I_{k}(f)=\sum_{k: k \in S} \hat{f}(S)^{2}$ and $I(f)=\sum_{S}|S| \hat{f}(S)^{2}$. It is easy to see that from the last part of the proof. Proposition 5.8 If $f: \Omega_{n} \rightarrow\{0,1\}$ is monotone, then for all $k$

$$
I_{k}=2 \hat{f}(\{k\})
$$

Proof. Note that

$$
\hat{f}(\{k\}):=\mathbb{E}\left[f \chi_{\{k\}}\right]=\mathbb{E}\left[f \chi_{\{k\}} \mathbb{1}_{k \notin \mathcal{P}}\right]+\mathbb{E}\left[f \chi_{\{k\}} \mathbb{1}_{k \in \mathcal{P}}\right]
$$

Note that the first term is always zero, while the second one is equal to $I_{k}(f) / 2$ due to monotonicity. So the result follows.

We remark that if $\mathrm{f}$ maps into $\{-1,1\}$ we may get by following the same proof that $I_{k}(f)=\hat{f}(\{k\})$. We also remark that the condition of monoticity is essential since for the Parity function $I_{k}(f)=1$ and $\hat{f}(\{k\})=0$.

\section{2}

\section{Noise Sensitivity}

In this section we will define the long-awaited notion of noise sensitivity. We will also introduce a few other notions and connect them with the energy spectrum as we did in the previous section.

Definição 5.9 Let $\omega$ be uniformly chosen from $\Omega_{n}$ and let $\omega_{\varepsilon}$ be $\omega$ but with each bit independently re-randomized with probability $\varepsilon$. This means that for each bit, independently, we will choose it with probability $\varepsilon$ and flip a coin with probability $1 / 2$ of giving 1 , and $1 / 2$ of giving -1 . Note that $\omega_{\varepsilon}$ has the same distribution as $\omega$. Now let $m_{n}$ be an increasing sequence of integers and let $f_{n}: \Omega_{m_{n}} \rightarrow\{-1,1\}$ or $\{0,1\}$. Then, $f$ is noise sensitive if for every $\varepsilon>0$

$$
\lim _{n \rightarrow \infty} \mathbb{E}\left[f_{n}(\omega) f_{n}\left(\omega_{\varepsilon}\right)\right]-\mathbb{E}\left[f_{n}(\omega)\right]^{2}=0
$$

Since $f_{n}$ takes the value, the definition says that the random variables $f_{n}(\omega)$ and $f_{n}\left(\omega_{\varepsilon}\right)$ are asymptotically independent for $\varepsilon$ fixed and $n$ large. When we connect this notion with the one of energy spectrum we will see that it is sufficient that such condition holds for one $\varepsilon \in(0,1)$.

We also state the notion of noise stability that captures an opposite situation 
Definição 5.10 The sequence $\left\{f_{n}\right\}$ is noise stable if

$$
\lim _{\varepsilon \rightarrow 0} \sup _{n} \mathbb{P}\left(f_{n}(\omega) \neq f_{n}\left(\omega_{\varepsilon}\right)\right)=0
$$

Even though they are opposed notions, a sequence of functions can be both noise sensitive and noise stable, but this happens if and only if $\operatorname{Var}\left(f_{n}\right) \rightarrow 0$. A sequence of functions can also be neither noise sensitive nor noise stable as the example below (check this!):

$$
G_{n}\left(x_{1}, \ldots, x_{n}\right)= \begin{cases}x_{1}, & \text { if } x_{1}=1 \\ \operatorname{Par}_{n-1}\left(x_{2}, \ldots, x_{n}\right), & \text { if } x_{1}=-1\end{cases}
$$

It is an easy exercise to check that Dictator is noise stable and Parity is noise sensitivity. Majority is also noise stable, and there is an important theorem that can be found in [13] that says in some sense that, among all low-influence Boolean functions, Majority is the stablest.

We also remark that this notions can be easily extended for $p \in[0,1]$. Just pick $\varepsilon$ with a $p$ probability coin. With that it is not easy to see that for $p \neq 1 / 2$ crossings on $\mathbb{Z}^{2}$ (or $\mathbb{T}$ ) are both noise sensitive and noise stable. In fact, since for $p>1 / 2 \mathbb{P}_{H_{k n, n}} \rightarrow 1$ and for $p<1 / 2 \mathbb{P}_{H_{k n, n}} \rightarrow 0$, we have in both cases that $\operatorname{Var}\left(f_{n}\right) \rightarrow 0$. The same thing won 't happen in the case $p=1 / 2$ thanks to the RSW theorem. This, will be in a fact a much more complicated case that will need extremely non trivial theorems such as the BKS Noise Sensitivity Theorem and SS Revealment Theorem. We will dedicate the next sections to prove them. Before we end this section we present the connection between noise sensitivity and the energy spectrum.

Proposition 5.11 A sequence of Boolean functions $f_{n}:\{-1,1\}^{m_{n}} \rightarrow\{-1,1\}$ or $\{0,1\}$ is noise sensitive if and only for any $k \geq 1$

$$
\sum_{m=1}^{k} \sum_{|S|=m} \hat{f}_{n}(S)^{2}=\sum_{m=1}^{k} E_{f_{n}}(m) \rightarrow 0, \text { as } n \rightarrow \infty .
$$

Moreover, to be noise sensitive does no depend on the value of $\varepsilon \in(0,1)$ chosen.

Proof. First note that for any $f: \Omega_{n} \rightarrow \mathbb{R}$ we have that

$$
\mathbb{E}\left[f(\omega) f\left(\omega_{\varepsilon}\right)\right]=\sum_{S} \hat{f}(S)^{2}(1-\varepsilon)^{|S|}
$$


so we may obtain that

$$
\operatorname{Cov}\left(f(\omega), f\left(\omega_{\varepsilon}\right)\right)=\sum_{m=1}^{n} E_{f}(m)(1-\varepsilon)^{m}
$$

Note that if $f_{n}$ is noise noise sensitive for a single $\varepsilon \in(0,1)$, then:

$$
\operatorname{Cov}\left(f_{n}(\omega), f_{n}\left(\omega_{\varepsilon}\right)\right)=\sum_{m=1}^{m_{n}} E_{f_{n}}(m)(1-\varepsilon)^{m} \rightarrow 0, \text { as } n \rightarrow \infty .
$$

But, for any fixed $k \geq 1$

$$
\sum_{m=1}^{k} E_{f_{n}}(m) \cdot \frac{1}{2^{k}} \leq \sum_{m=1}^{m_{n}} E_{f_{n}}(m)(1-\varepsilon)^{m}
$$

for all $m_{n} \geq k$. So, we must have that $\sum_{m=1}^{k} E_{f_{n}}(m) \rightarrow 0$ as $n \rightarrow \infty$.

In the other direction, we must note that for $f_{n}: \Omega_{m_{n}} \rightarrow\{-1,1\}$ or $\{0,1\}, \sum_{m=1}^{n} E_{f_{n}}(m) \leq 1$. Fix $\varepsilon \in(0,1)$. For any $\delta>0$, pick up $k(\delta)$ such that $\sum_{m=k}^{\infty}(1-\varepsilon)^{m}<\delta$. So, pick up $N$ such that for all $n \geq N, \sum_{m=1}^{n} E_{f_{n}}(m)<\delta$.

Then, we have that for all $n \geq N$

$$
\begin{aligned}
\operatorname{Cov}\left(f_{n}(\omega), f_{n}\left(\omega_{\varepsilon}\right)\right) & \leq \sum_{m=1}^{\infty} E_{f_{n}}(m)(1-\varepsilon)^{m} \\
& <\sum_{m=1}^{k} E_{f_{n}}(m)+\delta \\
& <2 \delta .
\end{aligned}
$$

Since $\delta$ and $\varepsilon$ are arbitrary, we have just proved that for all $\varepsilon \in(0,1)$, $\operatorname{Cov}\left(f_{n}(\omega), f_{n}\left(\omega_{\varepsilon}\right)\right) \rightarrow 0$. So, we conclude the proposition.

We present, without proof, a similar result to noise stability. From now on, we will only work with the notion of noise sensitivity.

Proposition 5.12 A sequence of Boolean functions $f_{n}:\{-1,1\}^{m_{n}} \rightarrow\{-1,1\}$ or $\{0,1\}$ is noise stable if and only for any $\varepsilon>0$, there exists $k$ such that for all $n$,

$$
\sum_{m=k}^{\infty} \sum_{|S|=m} \hat{f}_{n}(S)^{2}=\sum_{m=k}^{\infty} E_{f_{n}}(m)<\varepsilon
$$

And with that, we end this section. 


\section{3}

\section{The Benjamini-Kalai-Schramm Noise Sensitivity Theorem}

We now come to the main theorem regarding noise sensitivity.

Theorem 5.13 (BKS99) If

$$
\lim _{n \rightarrow \infty} \sum_{k=1}^{m_{n}} I_{k}\left(f_{n}\right)^{2}=0,
$$

then $\left\{f_{n}\right\}$ is noise sensitive.

We begin this by remarking that the converse is clearly not true. As it was noticed, Parity is noise sensitive but $\sum_{k=1}^{n} I_{k}\left(f_{n}\right)^{2}=n$. But, if $f_{n}$ is a sequence of monotone functions the converse turns out to be true. This is in fact easy to prove and may be derived by Propositions 5.8 and 5.11. Just observe that if $f_{n}: \Omega_{m_{n}} \rightarrow\{-1,1\}$ is a sequence of monotone functions, we have by Proposition 5.8 that

$$
\sum_{k=1}^{m_{n}} I_{k}\left(f_{n}\right)^{2}=\sum_{k=1}^{m_{n}} \hat{f}(\{k\})^{2} .
$$

If we suppose that $\left\{f_{n}\right\}$ is noise sensitive, we have by Proposition 5.11 that $E_{f_{n}} \rightarrow 0$ as $n$ tends to infinity. So, we may conclude that

$$
\lim _{n \rightarrow \infty} \sum_{k=1}^{m_{n}} I_{k}\left(f_{n}\right)^{2}=0,
$$

as required.

But, before we prove the BKS Theorem we will make two important remarks. The first one is that a very important tool is the Hypercontractivity Theorem. We won't prove it since it is a more technical result and it can be found in [9]. We first define the noise operator $T_{\rho}$.

Definição 5.14 For any $\rho \in[0,1]$, let $T_{\rho}$ be the following noise operator on the set of functions on the hypercube: For any $f: \Omega_{n} \rightarrow \mathbb{R}$, we define $T_{\rho} f: \omega \rightarrow \mathbb{E}\left[f\left(\omega_{1-\rho}\right) \mid \omega\right]$. It is not very hard to deduce from the definition $\widehat{T_{\rho} f}(S)=\mathbb{E}\left[T_{\rho} f \chi_{s}\right]$ that

$$
T_{\rho}: f=\sum_{S} \hat{f}(S) \chi_{S} \rightarrow \sum_{S} \rho^{|S|} \hat{f}(S) \chi_{S}
$$

Theorem 5.15 (Hypercontractivity) For any $f: \Omega_{n} \rightarrow \mathbb{R}$ and any $\rho \in$ $[0,1]$

$$
\left\|T_{\rho} f\right\|_{2} \leq\|f\|_{1+\rho^{2}}
$$


where $\|f\|_{1+\rho^{2}}:=\left(\sum_{\omega} \frac{|f(\omega)|^{1+\rho^{2}}}{2^{n}}\right)^{1 / 1+\rho^{2}}$.

The second remark is that we will assume a stronger condition but we will use the theorem in its most general form. The general proof is an extremely technical result that depends on estimates obtained by Talagrand. We refer the original paper [2] for more details. The stronger condition that we assume is namely that if

$$
H\left(f_{n}\right):=\sum_{k=1}^{m_{n}} I_{k}^{2},
$$

we require that $H\left(f_{n}\right) \leq\left(m_{n}\right)^{-\delta}$ for some exponent $\delta>0$. So, we are conditioning the decay to be polynomial. We actually can prove a more quantitative result:

Proposition 5.16 For any $\delta>0$, there exists a constant $M(\delta)>0$ such that if $f_{n}: \Omega_{m_{n}} \rightarrow\{0,1\}$ is any sequence of functions satisfying

$$
H\left(f_{n}\right) \leq\left(m_{n}\right)^{-\delta}
$$

then

$$
\sum_{1 \leq|S| \leq M \log \left(m_{n}\right)} \hat{f}_{n}(S)^{2} \rightarrow 0
$$

Note that with Proposition 5.11 this result implies the BKS Theorem when $H\left(f_{n}\right)$ decays as assume. We also observe that the same result may be achieved with $f: \Omega_{n} \rightarrow\{-1,1\}$, but we state at this way because our main application will be for the indicator of crossings.

Proof. Let $M>0$ and $\rho \in(0,1)$ be constants whose values will be chosen later. We have that:

$$
\begin{aligned}
\sum_{1 \leq|S| \leq M \log \left(m_{n}\right)} \hat{f}_{n}(S)^{2} & \leq 4 \sum_{1 \leq|S| \leq M \log \left(m_{n}\right.}|S| \hat{f}_{n}(S)^{2} \\
& =\sum_{k=1}^{m_{n}} \sum_{1 \leq|S| \leq M \log \left(m_{n}\right)} \widehat{\nabla_{k} f_{n}}(S)^{2} \\
& \leq \sum_{k}\left(\frac{1}{\rho^{2}}\right)^{M \log \left(m_{n}\right)} \| T_{\rho}\left(\nabla_{k} f_{n} \|_{2}^{2}\right. \\
& \leq \sum_{k}\left(\frac{1}{\rho^{2}}\right)^{M \log \left(m_{n}\right)}\left\|\nabla_{k} f_{n}\right\|_{1+\rho^{2}}^{2}
\end{aligned}
$$

where the last inequality follows from the Hypercontractivity Theorem. Now, since $\nabla_{k} f_{n} \in\{-1,0,1\}$, we have that $\left\|\nabla_{k} f_{n}\right\|_{1+\rho^{2}}=\left\|\nabla_{k} f_{n}\right\|_{2}^{2 /\left(1+\rho^{2}\right)}$. So, 


$$
\begin{aligned}
\sum_{1 \leq|S| \leq M \log \left(m_{n}\right)} \hat{f}_{n}(S)^{2} & \leq \sum_{k} \rho^{-2 M \log \left(m_{n}\right)}\left\|\nabla_{k} f_{n}\right\|_{2}^{4 /\left(1+\rho^{2}\right)} \\
& =\rho^{-2 M \log \left(m_{n}\right)} \sum_{k} I_{k}\left(f_{n}\right)^{2 /\left(1+\rho^{2}\right)} \\
& \leq \rho^{-2 M \log \left(m_{n}\right)}\left(m_{n}\right)^{\rho^{2} / 1+\rho^{2}} H\left(f_{n}\right)^{\frac{1}{1+\rho^{2}}}
\end{aligned}
$$

where the second inequality follows by Holder (??). Now, pick $\rho(\delta>0)$ sufficiently small such that $\frac{\rho^{2}-\delta}{1+\rho^{2}} \leq-\frac{\delta}{4}$. Also, pick up $M(\rho)=M(\delta)>0$ small such that $2 M \log \left(\rho^{-1}\right)<\delta / 8$. Then, we have that

$$
\sum_{1 \leq|S| \leq M \log \left(m_{n}\right)} \hat{f}_{n}(S)^{2} \leq\left(m_{n}\right)^{2 M \log \left(\rho^{-1}\right)} m_{n}^{\delta / 4} \leq m_{n}^{-\delta / 8} \rightarrow 0,
$$

and so we conclude the proof.

We remark that a proof of "Crossings are noise sensitive" can be obtained directly from this theorem. The original approach found in [2] obtained that by showing crossings on $\mathbb{Z}^{2}$ are very uncorrelated with the majority function, and showing that this implies that crossings are noise sensitive. A different approach done in [9] used the same theorem, but directly bounded the influences with critical exponents for the 4-arm events, also taking care with the boundary which require the analysis of different types of events, such as the 1 -arm event. But instead of taking this approaches we will introduce the idea of revealment and basically prove that $H(f) \leq \delta_{A}(f)$. So proving that $\delta_{A}\left(f_{n}\right) \rightarrow 0$, is enough to show that $\left\{f_{n}\right\}$ is noise sensitive.

\section{4}

\section{The Steif-Schramm Revealment Theorem}

Before we formally present the Revealment Theorem, we must first introduce a few notions.

An algorithm for a Boolean function $f$ is an algorithm $A$ that queries the values of the bits one by one, where the decision of which bit to ask can be based on the values of the bits previously queried, and it stops when the value of $f$ is determined (it doesn't depend on the remaining bits).

A randomized algorithm for a Boolean function $f$ is the same as above but we can use randomness to decide the next value to be queried.

Given a randomized algorithm $A$ for a Boolean function $f$, let $J_{A}$ denote the random set of bits queried by $A$.

Definição 5.17 (Revealment) The revealment of a randomized algo- 
rithm $A$ for a Boolean function $f$, denoted by $\delta_{A}(f)$, is defined by

$$
\delta_{A}:=\max _{i \in[n]} \mathbb{P}\left(i \in J_{A}\right) .
$$

The revealment of a Boolean function $f$, denoted by $\delta_{f}$, is defined by

$$
\delta_{f}=\inf _{A} \delta_{A}(f)
$$

where the infimum is taken over all randomized algorithms A for $f$.

Note that this notions can be immediately extended to functions $f: \Omega_{n} \rightarrow \mathbb{R}$. We may now present the main result of this section, proved in [15].

Theorem 5.18 (SS10) For any function $f: \Omega_{n} \rightarrow \mathbb{R}$ and for each $k \geq 1$, we have that

$$
E_{f}(k)=\sum_{|S|=k} \hat{f}(S)^{2} \leq \delta_{f} k\|f\|_{2}^{2}
$$

Note that for $f_{n}=\mathbb{1}_{H_{n+1, n}}$, if we find a random algorithm $A_{n}$, such that $\delta_{A_{n}}\left(f_{n}\right) \rightarrow 0$, we have that for each fixed $k, E_{f}(k) \rightarrow 0$. So we may use Proposition 5.11 to conclude that $\left\{f_{n}\right\}$ is noise sensitive. This is, indeed, an extremely powerful result that will also be used for our applications regarding Quenched Voronoi Percolation.

In the following proof, let $\widetilde{\Omega}$ denote the probability space that includes the randomness in the input bits of $f$ and the randomness used to run the algorithm (which we assume to be independent) and we let $\mathbb{E}$ be the corresponding expectation. Elements of $\widetilde{\Omega}$ may be represent without loss of generality as $\tilde{\omega}=(\omega, \tau)$ where $\omega$ are the random bits and $\tau$ represents the randomness necessary to run the algorithm.

Proof. Fix $k \geq 1$. Let

$$
g(\omega):=\sum_{|S|=k} \hat{f}(S) \chi_{S}(\omega)
$$

for all $\omega \in \Omega$. Note that $\|g\|_{2}^{2}:=\sum_{|S|=k} \hat{f}(S)^{2}$.

Now, let $J \subseteq[n]$ be the random set of all bits examined by the algorithm. Let $\mathcal{A}$ be the minimal $\sigma$-field for which $J$ is measurable and every $\omega_{i}, i \in J$, is measurable. This can be viewed as the relevant information gathered by the algorithm. For any function $h: \Omega \rightarrow \mathbb{R}$, let $h_{J}: \Omega \rightarrow \mathbb{R}$ denote the random function obtained by substituting the values of the bits in $J$. More precisely, if $\tilde{\omega}=(\omega, \tau)$ and $\omega^{\prime} \in \Omega$, then $h_{J}(\tilde{\omega})\left(\omega^{\prime}\right)$ is $h\left(\omega^{\prime \prime}\right)$ where $\omega^{\prime \prime}$ where $\omega^{\prime \prime}$ is $\omega$ on $J(\tilde{\omega})$ and is $\omega^{\prime}$ on $[n] \backslash J(\tilde{\omega})$. So, $h_{J}$ is a random variable on $\tilde{\Omega}$ taking values in the set of mappings from $\Omega$ to $\mathbb{R}$. Note that this random variable is $\mathcal{A}$-measurable. 
When the algorithm terminates, the unexamined bits in $\omega$ are free and hence, we must have that $\mathbb{E}[h \mid \mathcal{A}]=\int h_{J}=\hat{h_{J}}(\emptyset)$, where the integral is defined to be the integration with respect to the uniform measure on $\Omega$. It follows by the definition of conditional expectation that $\mathbb{E}[h]=\mathbb{E}\left[\int h_{J}\right]$. Analogously, for all $h$,

$$
\|h\|_{2}^{2}=\mathbb{E}\left[h^{2}\right]=\mathbb{E}\left[\int h_{J}^{2}\right]=\mathbb{E}\left[\left\|h_{J}\right\|_{2}^{2}\right] .
$$

Since the algorithm determines $f, f$ is $\mathcal{A}$-measurable, and so, by Theorem 2.2 properties, we have that:

$$
\|g\|_{2}^{2}=\mathbb{E}[g f]=\mathbb{E}[\mathbb{E}[g f \mid \mathcal{A}]]=\mathbb{E}[f E[g \mid \mathcal{A}]]
$$

Since $\mathbb{E}[g \mid \mathcal{A}]$, Holder's Inequality gives us that

$$
\|g\|_{2}^{2} \leq \sqrt{\mathbb{E}\left[\hat{g}_{J}(\emptyset)^{2}\right]}\|f\|_{2}
$$

Applying Parseval's formula to the random function $g_{J}$ gives us that for any $\tilde{\omega} \in \widetilde{\Omega}$,

$$
\hat{g}_{J}(\emptyset)^{2}=\left\|g_{J}\right\|_{2}^{2}-\sum_{|S|>0} \hat{g_{J}}(S)^{2}
$$

Taking the expectation over $\tilde{\omega} \in \widetilde{\Omega}$ gives us that

$$
\begin{aligned}
\mathbb{E}\left[\hat{g}_{J}^{2}\right] & =\mathbb{E}\left[\left\|g_{J}\right\|_{2}^{2}\right]-\sum_{|S|>0} \mathbb{E}\left[\hat{g}_{J}(S)^{2}\right] \\
& =\sum_{|S|=k} \hat{g}(S)^{2}-\sum_{|S|>0} \mathbb{E}\left[\hat{g}_{J}(S)^{2}\right] \\
& \leq \sum_{|S|=k} \mathbb{E}\left[\hat{g}(S)^{2}-\hat{g}_{J}(S)^{2}\right] .
\end{aligned}
$$

Now, since $g_{J}$ is built randomly from $g$ by fixing the variables in $J=J(\tilde{\omega})$, and since $g$ by definition does not have Fourier coefficients larger than $k$, for any $S$ with $|S|=k$ we have that

$$
\mathbb{E}\left[g_{J} \chi_{S}\right]=\hat{g}_{J}(S)= \begin{cases}\hat{g}(S)=\hat{f}(S), & \text { if } S \cap J(\tilde{\omega})=\emptyset \\ 0, & \text { otherwise. }\end{cases}
$$

So, we obtain that

$$
\begin{aligned}
\mathbb{E}\left[\hat{g}_{J}(\emptyset)^{2}\right] & \leq \sum_{|S|=k} \hat{g}(S)^{2} \mathbb{P}(S \cap J \neq \emptyset) \\
& \leq\|g\|_{2}^{2} k \delta_{A} .
\end{aligned}
$$


So, we have that

$$
\|g\|_{2}^{2} \leq\|g\|_{2}\|f\|_{2} \sqrt{k \delta_{A}}
$$

and by squaring both sides we finally conclude that

$$
\sum_{|S|=k} \hat{f}(S)^{2} \leq\|f\|_{2}^{2} k \delta_{A}
$$

Since $A$ was arbitrary, the same is true for $\delta_{f}$, and thus, we conclude the proof.

\section{5}

\section{Crossings are Noise Sensitive}

We will only do a sketch of the proof since the details are a require a little bit of extra work. We will also work with the hexagonal lattice since $\mathbb{T}^{*}=\mathbb{T}$ makes it easier to visualize the algorithm.

Theorem 5.19 Start by picking up $Q=Q_{R}$ to be the indicator function for the event that critical site percolation on the start hexagonal lattice contains a left to right open crossing in some grid-approximating domain $D$ to a large square of side length $R$. Then, there exists $\alpha>0$ such that $\delta_{A} \leq R^{-\alpha}$.

Proof. The algorithm proceeds as follows. There are four distinguished boundary $\operatorname{arcs}$ of $D$, which we call "left", "right", "up" and "down". Pick uniformly at random an edge $e_{0}$ on the right had boundary of $D$, and let $p_{0}$ be its midpoint. Let $\zeta$ be the union of top and left boundary segments of $D$. Explore the interface $\beta$, which has black hexagons to the right and white hexagons to the left, from $p_{0}$ to $\zeta$, examining the bits associated to sites in hexagons touching that interface, only as needed to continue the determination of the interface. This interface is uniquely defined: If the hexagon is white, then $\beta$ must take a $\pi / 3$ turn to the right, and if the hexagon is black, then $\beta$ must take a $\pi / 3$ turn to the left. See the image below

Now, let $\zeta^{\prime}$ be the union of the bottom and left boundaries of $D$, and $\beta^{\prime}$ that corresponds to the configuration $\omega^{\prime}$ obtained by flipping all the colors of the hexagons in $\bar{D}$.

Now, we need to calculate the revealment of this algorithm. Observe that for the points near the boundary, for instance, up to distance $\sqrt{R}$. Note that the probability of the random starting point $p_{0}$ is within distance $\sqrt{R}$ from the examined hexagon is $O(\sqrt{R} / R)$.

Observe that for hexagons at distance bigger than $\sqrt{R}$, there must exist a path of open (black) hexagons from the hexagon $H$ to the boundary of $D$ of size bigger than $\sqrt{R}$. But this is exactly the one arm event, that was actually 
already bounded for $\mathbb{Z}^{2}$ when we proved Theorem 3.13. We may do the same annulus method to bound such event and we may obtain $\alpha>0$ such that $\mathbb{P}\left(A_{\sqrt{R}}^{1}\right) \leq R^{-\alpha}$. With that we prove that

$$
\delta_{\mathcal{A}}\left(Q_{R}\right)=O\left(R^{-1 / 2}+R^{-\alpha}\right)
$$

and so we may conclude that $\delta_{Q_{R}} \rightarrow 0$. With the BKS and SS theorems, we prove that $\left\{Q_{R}\right\}$ is noise sensitive and we obtain the desired result.

Note that this weaker (since it is not interested in the best exponents) proof depends only on the RSW Theorem, and so generalizes easily to other models. With a little bit of care, the proof works for similar models provided we have an RSW result.

Doing this for Quenched Voronoi Percolation requires some more ideas alongside the ideas that we have seen above. The next section of this dissertation will be dedicated to how the authors (AGMT) achieved this result.

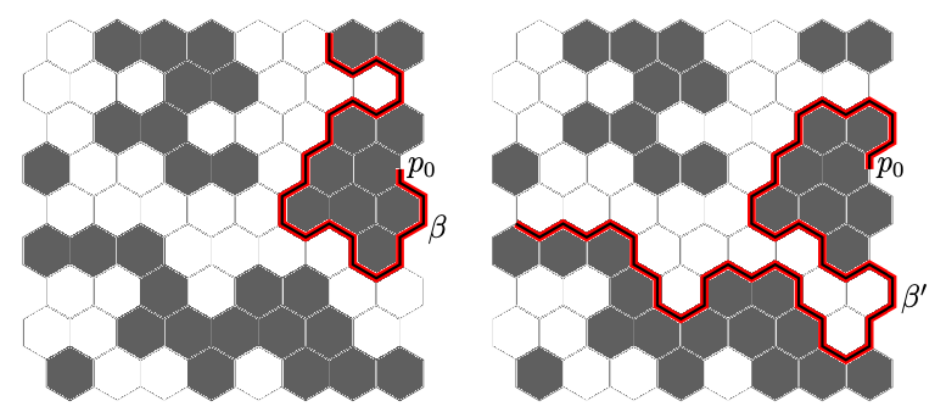

Figure 5.2: The interfaces $\beta$ and $\beta^{\prime}$. 


\section{6 \\ Quenched Voronoi Percolation}

This Chapter is devoted to a careful study of the paper "Quenched Voronoi Percolation" [1]. Though we can study quenched Voronoi Percolation for the $\mathbb{P}_{P_{o}}$ model, the original conjecture was stated for what we will call the Binomial model. This model actually creates some extra technicalities since it does not have the same independence property as the Poisson model.

Definição 6.1 (Binomial Model) We will consider a set $\eta$ of $n$ points in a rectangle $R$, each chosen independently and uniformly at random. For each $u \in \eta$, we define the Voronoi cell of $u$ to be

$$
V(u):=\left\{x \in R:\|u-x\|_{2} \leq\|v-x\|_{2} \text { for every } v \in \eta\right\}
$$

We then independently color each point $z \in \eta$, and its corresponding cell, red or blue each with probability $1 / 2$. This may be encoded as a function $\omega: \eta \rightarrow\{$ blue, red $\}$. We denote by $\mathbb{P}_{\operatorname{Bin}(n, R)}$ the probability measure associated with the selection of the pair $(\eta, \omega)$. In fact, sometimes it will be convenient to abuse notation and also use $\mathbb{P}_{B i n(n, R)}$ for the probability measure associated with selecting only $\eta$.

We focus on studying crossing probabilities in rectangles. Let $H_{R}$ denote the event that there is a red horizontal crossing in the rectangle $R$.

A straightforward duality argument (as in the Poisson case) shows that $\mathbb{P}_{\operatorname{Bin}(n, R)}\left(H_{R}\right)=1 / 2$ in the case that $H$ is a square. This event depends on the set of points $\eta$ and its coloring $\omega$. It is natural to ask which matters most: $\eta$ or $\omega$. Benjamini, Kalai and Schramm [2] conjectured that the point set $\eta$ does not matter much at all. Let $\mathbb{P}\left(H_{R} \mid \eta\right)$ be the conditional probability of the crossing given the point set $\eta$. They conjectured that if $\eta \sim \operatorname{Bin}(n, R)$ then $\mathbb{P}\left(H_{R} \mid \eta\right) \rightarrow 1 / 2$ in probability as $n \rightarrow \infty$.

We may now state the main theorem of [1] which confirms the conjecture in a strong sense.

Theorem 6.2 For all $\theta \in(0,1)$, there exists $N(\theta) \geq 1$ and $c>0$ such that the following holds. Let $R^{0}$ be an axis-parallel rectangle with aspect ratio 
$\rho \in(\theta, 1 / \theta)$. Then

$$
\mathbb{P}_{\operatorname{Bin}\left(n, R^{0}\right)}\left(\left|\mathbb{P}\left(H_{R^{0}} \mid \eta\right)-\mathbb{P}_{B i n\left(n, R^{0}\right)}\left(H_{R^{0}}\right)\right| \geq n^{-c}\right) \leq n^{-c},
$$

for every $n \geq N$.

Let us outline the four main steps of the proof. The first step, reproduced in Section 6.2, is to achieve a weaker bound for crossing probabilities, in the (quenched) Poisson model $\mathbb{P}_{P o}$ and the model $\mathbb{P}_{P o \mid \mathbb{H}}$ of (quenched) Poisson restricted to the half-plane; see below for a formal definition. Then, in Section 6.3 we translate this result to the (quenched) Binomial model $\mathbb{P}_{B i n(n, R)}$, and apply it, together with the annulus method, to achieve a bound for the probability of the one-arm event. In Section 6.4 we state an Efron-Stein type inequality, which relates variance and Influence, and therefore reduces proving Theorem 6.2 to controlling Influences. Finally, in Section 6.5, we shall see that Influences (in fact, the sum of squares of influences in the quenched model) may be controlled in terms of the revealment of a randomized algorithm. As in Section 5 the revealment itself is controlled by our bounds on the probability of the one-arm event.

What is actually beautiful, is that so many of the ideas we discussed while introducing Noise Sensitivity and Percolation are brought together in this proof. In particular, the annulus method (Chapter 3), the RSW theorem (Chapter 4) and the the Revealment Theorem (Chapter 5) all make an appearance in the proof of Theorem 6.2.

We will not reproduce the proofs of all the lemmas of [1]. In particular we state the results on crossing probabilities in the half-plane and an Efron-Stein type inequality without proof.

On the other hand, there is one result in particular which we shall prove with much more detail than appears in [1]. This result, Proposition 3.11 of [1] (which appears as Proposition 6.15 here), bounds the probability of the one-arm event in the (quenched) Binomial model $\mathbb{P}_{\operatorname{Bin}(n, R)}$. There is a moment in the proof of Proposition 3.11 in [1] where a result proved for the Poisson model is applied in the Binomial model. This is certainly a problem. However, we shall see that it is not a major problem. We shall explain how, in certain circumstances, results for one model imply results for the other. The relevant results for translating between these models are Lemma 6.12 and Lemma 6.24. See also Lemma 7.5, in Chapter 7.

We remark that we state and prove certain results for axis-parallel rectangles. This is simply because it turns the drawings or visualization easier. But everything can be done for rectangles in general. 
Before we begin the proof we must introduce variants of the Poisson model to deal with boundary effects.

\section{1}

\section{Two more Poisson models (to deal with boundary problems)}

In this section we will present two more models that we will use alongside the $\mathbb{P}_{\text {Po }}$ model presented in Chapter 4 . We do them in a separate section to avoid confusion. These models are quite important to deal with boundary problems that naturally arises when we are dealing with the Binomial distribution. We will, frequently, throughout this thesis reproduce results for both $\mathbb{P}_{P_{o}}$ and $\mathbb{P}_{P o \mid H_{H}}$ (see the definition below) and sometimes, such as in Section 6.2 this can be a little bit tricky.

Definição 6.3 (Poisson Model on the Half plane) We may consider a Poisson Percolation Process restricted to the half plane $\mathbb{H}:=\left\{x \in \mathbb{R}^{2}: x \geq 0\right\}$ just by restricting the Voronoi cells to the half plane. Let $\left.u \in \eta\right|_{\mathbb{H}}$ :

$$
V(u):=\left\{x \in \mathbb{H}:\|u-x\|_{2} \leq\|v-x\|_{2} \text { for every }\left.v \in \eta\right|_{\mathbb{H}}\right\},
$$

where $\eta$ is naturally distributed as a Poisson Point Process. The associated probability measure to the Percolation Process is denoted by $\mathbb{P}_{\left.P o(\lambda)\right|_{\mathbb{H}}}$. We reduce it to $\mathbb{P}_{P o \mid \mathbb{H}}$ when $\lambda=1$. In fact, this will be our only case of interest.

Definição 6.4 (Poisson Model on the Quarter plane) Just as we did before, but instead, we restrict our process to the quarter plane $\mathbb{Q}:=\left\{\left(x_{1}, x_{2}\right) \in\right.$ $\left.\mathbb{R}^{2}: x_{1} \geq 0, x_{2} \geq 0\right\}$. We denote the Percolation Process associated with it by $\mathbb{P}_{P o \mid Q}$.

Now that the models have been properly introduced, we remark in a, yet, informal way, how they connect with the Binomial model. The problem is that while for events $E$ at the center of $R, \mathbb{P}_{B i n(n, R)} E$ behaves like $\mathbb{P}_{P o}(E)$, the same won't be true for events near the boundary, since it will influence the

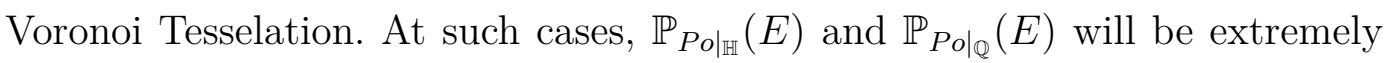
useful. More precisely, $\mathbb{P}_{P o \mid \mathbb{H}}(E)$ is useful for events close to only one border and $\mathbb{P}_{P o \mid \mathbb{Q}}(E)$ for events in the corner of the rectangle.

We also remark that for a rectangle $R \subset \mathbb{R}^{2}$ we denote by $R_{n}$, the rectangle with the same aspect ratio and center of $R$, but renormalized to have area $n$. We also denote by $\tau . R$, for $\tau>0$, the rectangle with the same center as $R$, but with $\tau$ times each side length of it. 


\section{2}

\section{Weak bounds for crossing probabilities on Quenched Voronoi Percolation}

In this section we will prove a weaker version of Theorem 6.2 on the "Poisson Model" and the "Poisson Model on the Half plane".

In this section, we will denote $\omega: \eta \rightarrow\{-1,1\}$ to be a uniform random two-coloring of $\eta$. Observe, that since, in this section, $\eta$ is distributed accordingly to a Poisson distribution (restricted to the half plane or not), $\omega$ is countable. But we remark that since we are only interested in the restriction of $\omega$ to $\eta_{R}$, the subset of points whose cells intersect a given rectangle $R$, which is almost surely finite.

Theorem 6.5 For every axis-parallel rectangle $R \subset \mathbb{R}^{2}$ with aspect ratio $\rho>0$, there exists a constant $\phi(\rho)>0$, that only depends on the aspect ratio of $R$, such that

$$
\mathbb{P}_{P o}\left(\mathbb{P}\left(H_{R} \mid \eta\right) \leq \frac{1}{2^{k}}\right) \leq(1-\phi)^{k},
$$

for all sufficiently large $k \geq N_{0}(\rho)$.

We begin by defining the following random variable $X=X(\eta, \omega)$, whose value depends on both $\eta$ and $\omega$, and which counts the maximum number of vertex-disjoint (no two of the crossings use points in the interior of the same Voronoi cell) vertical crossings of $R$ :

$$
\begin{gathered}
X:=\max _{m \in \mathbb{N}}\{\text { There exist } m \text { vertex-disjoint, monochromatic vertical crossings } \\
\left.\left\{\gamma_{1}, \ldots, \gamma_{m}\right\} \text { of } R\right\} .
\end{gathered}
$$

The following lemma will be a key tool in our proof of Theorem 6.5.

Lemma 6.6 (Color Switching Lemma) For almost every $\eta$,

$$
\mathbb{P}\left(H_{R} \mid \eta\right)=\mathbb{E}\left[2^{-X} \mid \eta\right]
$$

This follows almost immediately from a basic fact about "color-switching". For those unfamiliar with color-switching, we will give a brief introduction to it. Proof. Consider the event that $X=k$. This means that there exist $k$, but not $k+1$, vertex-disjoint, monochromatic vertical crossings of $R$. The following algorithm provides a method to find such paths. First, we discover the leftmost monochromatic path (in the colored Voronoi tiling of $R$ given by the pair $(\eta, \omega))$ by starting from the left-most cell which intersects the lower side of the rectangle and repeatedly exploring the left-most cell of this color, until either the explored component reaches the top of the rectangle (and then we add 
this monochromatic path to our collection) or we have exhausted the entire monochromatic component of the start point. In either case, we may start again from the next available cell on the lower side of $R$ (if it exists) and discover the left-most monochromatic path entirely to the right of the alreadydiscovered cells. Repeating this process until we reach the right-side of $R$, we obtain a collection $\left(\gamma_{1}, \ldots, \gamma_{k}\right)$ of disjoint monochromatic crossings.

An important feature of this algorithm is that it allows us to use "colorswitching" arguments. The fundamental observation, is that, for a given $\eta$, and a given collection of paths $\left(\gamma_{1}, \ldots, \gamma_{k}\right)$ obtained via the algorithm, there is a bijection between the set of colorings in which $\gamma_{j}$ is red, and those in which it is blue. Indeed, if we swap the color of all cells that are on or to the right of $\gamma_{j}$, then the algorithm produces exactly the same set of paths. More generally, if $\Pi \in\{-1,1\}^{k}$ denotes the sequence of colors of the paths $\left(\gamma_{1}, \ldots, \gamma_{k}\right)$, we have the following fact: For every $\sigma \in\{-1,1\}^{k}$, we have that

$$
\mathbb{P}(\Pi=\sigma \mid X=k, \eta)=\frac{1}{2^{k}}
$$

almost surely. Observe now, that the event $H_{R}$ holds if and only if all monochromatic paths are red. So, by what was done above

$$
\begin{aligned}
\mathbb{P}\left(H_{R} \mid \eta\right) & =\sum_{k=0}^{\infty} \mathbb{P}(\Pi=(1, \ldots, 1) \mid X=k, \eta) \mathbb{P}(X=k \mid \eta) \\
& =\sum_{k=0}^{\infty} \frac{\mathbb{P}(X=k \mid \eta)}{2^{k}}=\mathbb{E}\left[2^{-X} \mid \eta\right] .
\end{aligned}
$$

In order to obtain the proof of Theorem 6.5 we only need to show that $X$ cannot be too large. This will be a consequence of the FKG Inequality for annealed Voronoi Percolation (A.12), BK Inequality for annealed Voronoi Percolation (A.13) and the RSW Theorem for annealed Voronoi Percolation (4.5). We will state them again, in the exact form that they will be used here.

Lemma 6.7 (FKG Inequality for annealed Voronoi Percolation) Let $A$ and $B$ be red-increasing events. Then

$$
\mathbb{P}_{\text {Po }}(A \cap B) \geq \mathbb{P}_{\text {Po }}(A) \cdot \mathbb{P}_{\text {Po }}(B)
$$

Lemma 6.8 Let $A$ and $B$ be red-increasing events. Then:

$$
\mathbb{P}_{\text {Po }}(A \circ B) \leq \mathbb{P}_{\text {Po }}(A) . \mathbb{P}_{\text {Po }}(B),
$$


where $A \circ B$ denotes the event that $A$ and $B$ occurs disjointly. We define it formally in the appendix, but for events involving crossing the definition is straightforward: The crossings must be vertex-disjoint.

Theorem 6.9 (RSW for annealed Voronoi Percolation) There exists a constant $c_{0}$, depending only on the aspect ratio of $R$, such that

$$
c_{0} \leq \mathbb{P}_{P o}\left(H_{R}\right) \leq 1-c_{0}
$$

Proof.[Proof of Theorem 6.5] Given the pair $(\eta, \omega)$, define $X^{+}$, and $X^{-}$ respectively, to be the maximal number of disjoint red (respectively blue) TB crosses in $R$, so in particular $X=X^{+}+X^{-}$. By the RSW Theorem and the BK inequality, there exists a constant $c_{0}$, depending only on the aspect ratio of $R$, such that

$$
\mathbb{P}_{P o}\left(X^{-} \geq k\right) \leq\left(1-c_{0}\right)^{k}
$$

for all $k \geq 0$, and analogously $\mathbb{P}_{P_{o}}\left(X^{+} \geq k\right) \leq\left(1-c_{0}\right)^{k}$. Since the events $\left\{X^{+} \geq i\right\}$ and $\left\{X^{-}<j\right\}$ are both red-increasing, it follows by the FKG inequality that

$$
\begin{aligned}
\mathbb{P}_{\text {Po }}(X \geq k) & \leq \sum_{i+j \geq k} \mathbb{P}_{\text {Po }}\left(\left\{X^{+} \geq i\right\} \cap\left\{X^{-} \geq j\right\}\right) \\
& \leq \sum_{i+j \geq k}\left(1-c_{0}\right)^{i+j}=\frac{\left(1-c_{0}\right)^{k}}{c_{0}}(k+1)+\frac{\left(1-c_{0}\right)^{k+1}}{c_{0}^{2}} \\
& \leq \frac{(1-\phi)^{k}}{2}
\end{aligned}
$$

for some constant $\phi\left(c_{0}\right)>0$ and all sufficiently big $k \geq N_{0}\left(c_{0}\right)$. Now, by the Color-Switching Lemma we obtain that

$$
\mathbb{P}\left(H_{R} \mid \eta\right)=\mathbb{E}\left[2^{-X} \mid \eta\right] \geq \frac{1}{2^{k-1}} \mathbb{P}(X<k \mid \eta)
$$

almost surely. Thus, by Markov's (A.5) and the previous inequalities:

$$
\begin{aligned}
\mathbb{P}_{P o}\left(\mathbb{P}\left(H_{R} \mid \eta\right) \leq \frac{1}{2^{k}}\right) & \leq \mathbb{P}_{P o}\left(\mathbb{P}(X \geq k \mid \eta) \geq \frac{1}{2}\right) \\
& \leq 2 \mathbb{E}_{P o}[\mathbb{P}(X \geq k \mid \eta)] \\
& \leq(1-\phi)^{k}
\end{aligned}
$$

Since $c_{0}$ only depends on $\rho$ by the RSW Theorem, we get the desired result. 
As it was explained, for one-arm events of points near the boundary, a version of Theorem 6.5 for the Poisson Model restricted to half plane is also needed:

Theorem 6.10 For every axis-parallel rectangle $R \subset \mathbb{H}$, with aspect ratio $\rho>0$, there exists a constant $\phi^{*}(\rho)>0$, such that

$$
\mathbb{P}_{\left.P o\right|_{\mathbb{H}}}\left(\mathbb{P}\left(H_{R} \mid \eta\right) \geq \frac{1}{2^{k}}\right) \leq\left(1-\phi^{*}\right)^{k}
$$

for all $k \geq N_{0}^{*}(\rho)$.

To prove this, we actually need a version of the RSW Theorem for annealed Voronoi Percolation restricted to the half plane. Since this will be quite useful later, we will also state it as a theorem

Theorem 6.11 For every rectangle $R \subset \mathbb{H}$, there exists a constant $c_{1}>0$, depending only on the aspect ratio of $R$, such that

$$
c_{1}<\mathbb{P}_{P o \mid \mathbb{H}}\left(H_{R}\right)<1-c_{1} .
$$

If one has Theorem 6.11, the proof of Theorem 6.10 follows in the same way by replacing the RSW Theorem for annealed Voronoi Percolation with this version. Since this result requires a more complicated proof, we will refer to the paper for more details on it.

\section{3}

\section{A bound on the probability of the $1-$ arm event}

In this section we will bound the probability of the one arm event for the quenched $\mathbb{P}_{\text {Bin }(n, R)}$ model. We do so using the annulus method. For this reason we require some weak lower bound on crossing probabilities in the quenched model. We prove such bounds by comparison to the results proved for the Poisson Models above.

In translating from the Poisson models, we select the model $\mathbb{P}_{\text {Po }}$ for events strictly in the interior, the model $\mathbb{P}_{P o \mid \mathbb{H}}$ for events overlapping one boundary, and the model $\mathbb{P}_{P o \mid \mathbb{Q}}$ for events in corners.

Lemma 6.12 (Far from the boundary events) Let $R_{\alpha n}^{\prime}$ be an axisparallel rectangle (with area $\alpha n$ ) strictly contained in the interior of $R_{n}$ (a rectangle of area $n$ ), where $0<\alpha \leq \frac{1}{4}$. Let $E \in \mathcal{F}_{R_{\alpha n}^{\prime}}$. Then

$$
\mathbb{P}_{\operatorname{Bin}\left(n, R_{n}\right)}(E) \leq 3 \mathbb{P}_{P o}(E) .
$$




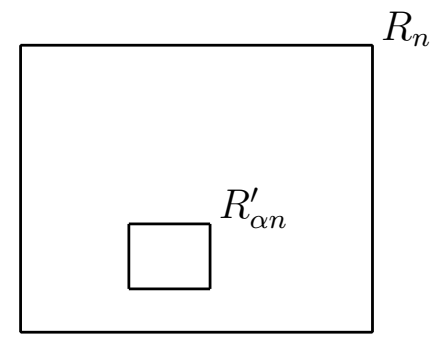

Figure 6.1: The event $E$ depends solely on the colors of the cells whose points lies inside $R_{\alpha n}^{\prime}$.

Proof. Let $R_{n}$ and $R_{\alpha . n}^{\prime}$ be fixed. Now, note that since $E \in \mathcal{F}_{R^{\prime} \alpha . n}$ :

$$
\begin{aligned}
\mathbb{P}_{\text {Po }}(E) & =\sum_{m \geq 0} \mathbb{P}_{\text {Po }}\left(E|| \eta \cap R_{\alpha n}^{\prime} \mid=m\right) \mathbb{P}_{\text {Po }}\left(\left|\eta \cap R_{\alpha n}^{\prime}\right|=m\right) \\
& =\sum_{m \geq 0} \mathbb{P}_{\left.P o\right|_{R_{n}}}\left(E|| \eta \cap R_{n}^{\alpha \prime} \mid=m\right) \mathbb{P}_{\left.P o\right|_{R_{n}}}\left(\left|\eta \cap R_{\alpha n}^{\prime}\right|=m\right) .
\end{aligned}
$$

We also have that:

$$
\begin{aligned}
\mathbb{P}_{\operatorname{Bin}\left(n, R_{n}\right)}(E) & =\sum_{m=0}^{n} \mathbb{P}_{\operatorname{Bin}\left(n, R_{n}\right)}\left(E|| \eta \cap R_{\alpha n}^{\prime} \mid=m\right) \mathbb{P}_{\operatorname{Bin}\left(n, R_{n}\right)}\left(\left|\eta \cap R_{\alpha n}^{\prime}\right|=m\right) \\
& =\sum_{m=0}^{n} \mathbb{P}_{\left.P\right|_{R_{n}}}\left(E|| \eta \cap R_{\alpha n}^{\prime} \mid=m\right) \mathbb{P}_{\operatorname{Bin}\left(n, R_{n}\right)}\left(\left|\eta \cap R_{\alpha n}^{\prime}\right|=m\right) .
\end{aligned}
$$

Now, we want want to compare $x_{m}=\mathbb{P}_{P_{\left.o\right|_{n}}}\left(\left|\eta \cap R_{\alpha n}^{\prime}\right|=m\right)$ with $\sigma_{m}=\mathbb{P}_{\operatorname{Bin}\left(n, R_{n}\right)}\left(\left|\eta \cap R_{\alpha n}^{\prime}\right|=m\right)$. So, we make the following claim:

Claim: We have that for every $0 \leq m \leq n$ :

$$
\frac{\sigma_{m}}{x_{m}} \leq 3
$$

Proof. First, remind that $A_{R_{\alpha . n}^{\prime}} \leq \frac{n}{4}$. The case $m=0$ is clearly bounded, since $(1-\alpha) \cdot e^{\alpha}<1$. So, by Stirling's approximation, for every $1 \leq m \leq 3 n / 4$ we have that:

$$
\sigma_{m}=\left(\begin{array}{c}
n \\
m
\end{array}\right)(\alpha)^{m}(1-\alpha)^{n-m} \leq \frac{e}{2 \pi} \frac{n^{n+1 / 2}(\alpha)^{m}(1-\alpha)^{n-m}}{m^{m+1 / 2}(n-m)^{n-m+1 / 2}}
$$

On the other hand, we have that :

$$
x_{m}=\frac{(\alpha n)^{m} e^{-\alpha n}}{m !} \geq \frac{(\alpha n)^{m} e^{-\alpha n}}{m^{m+1 / 2} e^{-m+1}} .
$$


So, by combining both inequalities, we get that

$$
\frac{\sigma_{m}}{x_{m}} \leq \frac{e^{2}}{2 \pi} \frac{(1-\alpha)^{n-m} e^{-m}}{e^{-\alpha n}}\left(\frac{n}{n-m}\right)^{n-m+1 / 2}
$$

Now, we may observe that the function on the right hand side has a natural continuous extension. Note that for every $m \leq 3 n / 4, m=\beta n$ for some $\beta \in[0,3 / 4]$. So we may define $h:[0,3 / 4] \rightarrow \mathbb{R}^{+}$as:

$$
\begin{aligned}
h(\beta) & :=\frac{e^{2}}{2 \pi \sqrt{1-\beta}}\left(1-\frac{\alpha-\beta}{1-\beta}\right)^{(1-\beta) n} e^{(\alpha-\beta) n} \\
& \leq \frac{e^{2}}{2 \pi \sqrt{1-\beta}} \leq 3 .
\end{aligned}
$$

For $m>3 n / 4$, it is easy to observe that we are multiplying the ratio by $\frac{1}{1-\alpha} \frac{(n-m)}{n}<1 / 3$. So,

$$
\frac{\sigma_{\lfloor 3 n / 4\rfloor}}{x_{\lfloor 3 n / 4\rfloor}} \geq \frac{\sigma_{m}}{x_{m}}
$$

So, by the Equation 1 together with the previous claim:

$$
\begin{aligned}
\mathbb{P}_{B i n\left(n, R_{n}\right)}(E) & \leq 3 \sum_{m=0}^{n} \mathbb{P}_{\left.P o\right|_{n}}\left(E|| \eta \cap R_{\alpha n}^{\prime} \mid=m\right) \mathbb{P}_{\left.P o\right|_{n}}\left(\left|\eta \cap R_{\alpha n}^{\prime}\right|=m\right) \\
& \leq 3 \mathbb{P}_{P o}(E) .
\end{aligned}
$$

We will state two analogous results. The reader shall note that the proof is carried in the exact same way. Since we don't want to be repetitive, we will just state them.

Lemma 6.13 (Close to one boundary events.) Let $R_{\alpha n}^{\prime}$ be an axisparallel rectangle,that has one of its sides "glued" to one of $R_{n}$ sides, where $0<\alpha \leq \frac{1}{4}$. Let $E \in \mathcal{F}_{R_{\alpha n}^{\prime}}$. Then

$$
\mathbb{P}_{B i n\left(n, R_{n}\right)}(E) \leq 3 \mathbb{P}_{P o \mid \mathbb{H}}(E)
$$

The reader may note, that the exact same result will also hold for $\mathbb{P}_{\text {Po }}(E)$. So why we do it for the process restricted to the half-plane? In the practical application, no event will exactly be measurable to $\mathcal{F}_{R_{\alpha n}^{\prime}}$ when it is glued to the left side. What happens is that it will depend on the color of the points slightly out of the boundary of the rectangle $R_{n}$. But, if we consider this side of the rectangle to coincide with the boundary of the half-plane (just 


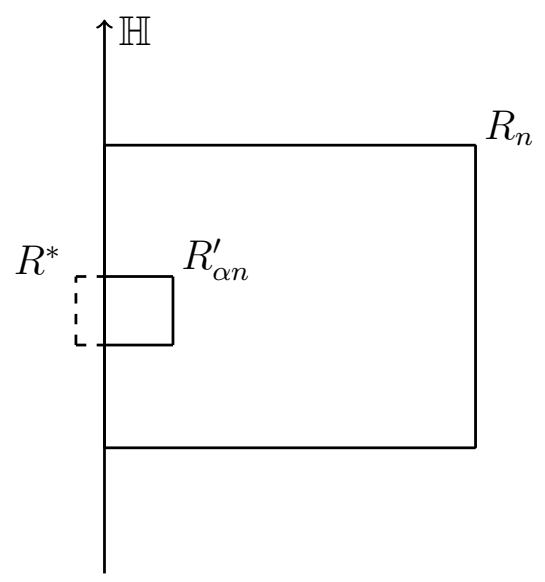

Figure 6.2: The event $E$ depends solely on the colors of the cells whose points lies inside $R_{\alpha n}^{\prime}$. The rectangle $R^{*}$ is the union of $R_{\alpha n}^{\prime}$ with the dashed lines. As pointed out, in the practical application, our event will be $\mathcal{F}_{R^{*}}$-measurable. But, when we restrict to $\mathbb{H}$ it is, indeed, $\mathcal{F}_{R_{\alpha n}^{\prime}}-$ measurable. The line with the arrow represents the division of the half-plane.

turn your heads!), the restriction of the process to the half-plane will be $\mathcal{F}_{R_{\alpha n}^{\prime}}-$ measurable as it is shown in the image above.

Lemma 6.14 (Close to two boundary events.) Let $R_{\alpha n}^{\prime}$ be an axisparallel rectangle,that has two of its sides "glued" to two of $R$ sides, where $0<\alpha \leq \frac{1}{4}$. Let $E \in \mathcal{F}_{R_{\alpha n}^{\prime}}$. Then

$$
\mathbb{P}_{\operatorname{Bin}\left(n, R_{n}\right)}(E) \leq 3 \mathbb{P}_{\text {Po| }}(E)
$$

A similar phenomena will happen, but now, with the quarter-plane. We express it in the drawing below:

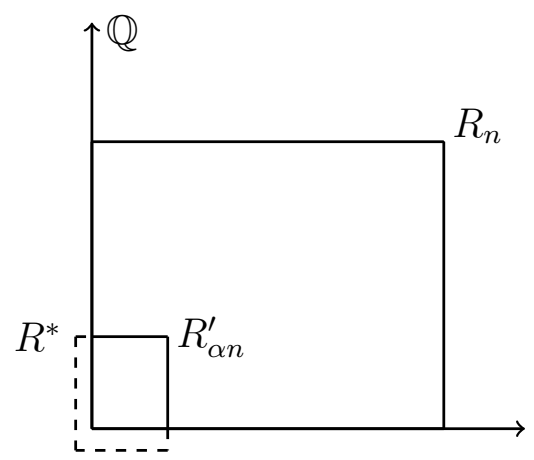

Figure 6.3: The event $E$ depends solely on the colors of the cells whose points lies inside $R_{\alpha n}^{\prime}$. As pointed out, in the practical application, our event will be $\mathcal{F}_{R^{*}}$-measurable. But, when we restrict to $\mathbb{Q}$ it is, indeed, $\mathcal{F}_{R_{\alpha n}^{\prime}}$-measurable. The lines with arrows represents the division of the quarter-plane.

We can now turn ourselves back to bounding the 1-arm event. Given 
$u \in R$ and $d>0$, we write $V(u, d)$ for the event (depending on $\eta$ and $\omega$ ) that there is a red path from $u$ to some point of $R$ at $l_{2}$-distance $d$ from $u$.

In fact we shall focus on the distance $\beta n^{1 / 4}$, where $\beta=\rho^{-1 / 2}$. This is a convenient choice for the required application (in the proof of Lemma 6.20), but of course the result also holds for any distance $d$ at least polynomial in $n$.

Proposition 6.15 For every $\gamma>0$, there exists $\varepsilon>0$ such that the following holds. Let $R^{0}$ be an axis-parallel rectangle with aspect ratio $\rho>0$. Let $\beta:=1 / \sqrt{\rho}$. Let $u \in R_{n}^{0}$. Then, there exists $N_{2}(\rho, \gamma)$ such that

$$
\mathbb{P}_{\operatorname{Bin}\left(n, R_{n}^{0}\right)}\left(\mathbb{P}\left(V\left(u, \beta n^{1 / 4}\right) \mid \eta\right) \geq n^{-\varepsilon}\right) \leq n^{-\gamma}
$$

for all $n \geq N_{2}$.

Proof. Fix a point $u \in R_{n}^{0}$. For each $j \in \mathbb{N}$, let $\mathcal{A}_{j}$ denote the square annulus, centered on $u$, with inner side-length $7^{j}$ and outer side-length $3.7^{j}$. Let $O_{j}$ denote the event that there is a blue circuit around the annulus (that may be partial, in the sense that it may both its endpoints on the boundary of $R$, separating $u$ from the exterior of $A_{j}$. We will divide this proof in three cases

Case 1: The distance from $u$ to any side of $R_{n}^{0}$ is bigger than $n^{1 / 7}$. In this case we will be working with $\mathbb{P}_{\text {Po }}$. Let

$$
J=\left\{j \in \mathbb{N}: n^{1 / 10} \leq 7^{j+1} \leq n^{1 / 9}\right\},
$$

and consider the collection of annulus $\mathcal{C}=\left\{\mathcal{A}_{j}: j \in J\right\}$. Let $\phi>0$ be the constant of Theorem 6.5 corresponding to the aspect ratio $\rho=3$ (rectangles $3: 1)$. Fix $k \in \mathbb{N}$ depending on $\phi$ and $\gamma$. For each $j \in J$, let $D_{j}^{(1)}$ denote the event (depending on $\eta$ ) that $\mathbb{P}\left(O_{j} \mid \eta\right)>2^{-4 k}$. Now, let $D_{j}^{(2)}$ denote the event that for every $z \in \mathcal{A}_{j}$, there exists some point $x \in \eta$ ( $\eta$ chosen accordingly to the Poisson distribution) at distance at most $n^{\frac{1}{32}}$ from $z$. Finally, define:

$$
D_{j}:=D_{j}^{(1)} \cap D_{j}^{(2)},
$$

and note that the events $D_{j}$ are independent with respect to the measure $\mathbb{P}_{P o}$. This is due to the fact that each $D_{j}^{(2)}$ depends on a restriction $\eta_{j}^{\prime}$ of $\eta$ to a certain neighborhood of $\mathcal{A}_{j}$. Since the distance between the annulus $\mathcal{A}_{j}$ and $\mathcal{A}_{j+1}$ is $4.7^{j}$, which is bigger than $n^{1 / 10}$ and we picked our distance on $D_{j}^{(2)}$ to be at most $n^{1 / 32}$, we will have that $\eta_{j}^{\prime}$ and $\eta_{j+1}^{\prime}$ are independent. So, by the properties of the Poisson distribution, those events are disjoint. Next, observe that

$$
\mathbb{P}_{\text {Po }}\left(D_{j}^{(2)}\right) \geq 1-e^{-n^{\frac{1}{32}}} .
$$


Next, observe that, by the FKG inequality and Theorem 6.5 , we have that

$$
\mathbb{P}_{\text {Po }}\left(\mathbb{P}\left(O_{j} \mid \eta\right) \leq 2^{-4 k}\right) \leq 4(1-\phi)^{k}
$$

for all $k \geq N_{0}$. So, $\mathbb{P}_{P o}\left(D_{j}^{c}\right) \leq 5(1-\phi)^{k}$, for all $n \geq N_{2}(\phi, k)$. Since $k$ depends on $\gamma$ and $\phi$ is a fixed constant, one may note that $N_{2}(\gamma)$. Now, let $D^{*}$ denote the event that $D_{j}$ holds for at least half of the elements $j \in J$, and observe that

$$
\mathbb{P}_{P o}\left(D^{*}\right) \geq 1-2^{|J|}\left(5(1-\phi)^{k}\right)^{|J| / 2} \geq 1-n^{-2 \cdot \gamma},
$$

since $|J| \geq \log (n) / 90$, and $k$ was chosen sufficiently large in terms of $\gamma$ and $\phi$. We now, observe that $D^{*} \in \mathcal{F}_{R^{\prime}}$ for the rectangle $R^{\prime}$ strictly contained in $R_{n}^{0}$, with area smaller than $n / 4$, since as pointed out, $D^{*}$ depends on the points contained in the union of the restrictions $\bigcup_{j \in J} \eta_{j}^{\prime}$ defined above (observe that the way we chose the distances and annulus size implies an existence of such $R^{\prime}$ that contains all those points). So, by Lemma 6.12 we actually have that

$$
\mathbb{P}_{\operatorname{Bin}\left(n, R_{n}^{0}\right)}\left(D^{*}\right) \geq 1-n^{-\gamma}
$$

for all $n \geq N_{2}(\gamma)$. Finally, for those $\eta$ such that $D^{*}$ holds, we get that

$$
\mathbb{P}_{B i n\left(n, R_{n}^{0}\right)}\left(V\left(u, n^{\frac{1}{4}} \mid \eta\right)\right) \leq \mathbb{P}\left(\bigcap_{j \in J} O_{j}^{c} \mid \eta\right)=\prod_{j \in J} \mathbb{P}\left(O_{j}^{c} \mid \eta\right) \leq\left(1-2^{-4 k}\right)^{|J| / 2} \leq n^{-\epsilon}
$$

for some $\varepsilon(\gamma)>0$ and $n \geq N_{2}(\gamma)$.

Case 2: The distance from $u$ to any side of $R_{n}^{0}$ is smaller than $n^{1 / 7}$ from one side and bigger than $n^{1 / 5}$ from the rest. In this case we will be working with $\mathbb{P}_{\left.P o\right|_{\mathbb{H}}}$. Let

$$
J=\left\{j \in \mathbb{N}: n^{1 / 7} \leq 7^{j+1} \leq n^{1 / 6}\right\}
$$

and consider the collection of partial annulus (just one of the rectangles that composes the annulus will be out of $R) \mathcal{C}=\left\{\mathcal{A}_{j}: j \in J\right\}$. In this case, the proof will carry out in the exact same way as in Case 1 , using Theorem 6.10 (that may give a different constant $\phi^{*}$, which won't be a problem) to reach that $\mathbb{P}_{P o \mid \mathbb{H}}\left(D^{*}\right) \geq 1-n^{-2 \cdot \gamma}$ for all $n \geq N_{2}(\gamma)$. But, as previously remarked, we will have to consider the half-plane glued to the closest side of $u$. So, when we restrict the events $D_{j}$ to $\mathbb{H}$, we may define similarly the restrictions $\eta_{j}^{\prime}$ of $\eta$ whose points define the events $D_{j}$ and are disjoint. We can also define a rectangle $R^{\prime}$ glued to the side of the rectangle which coincides with the division of $\mathbb{H}$ that satisfies that $\left.D^{*}\right|_{\mathbb{H}} \in \mathcal{F}_{R^{\prime}}$ and has area less than $n / 4$. So, we will be able to conclude that $\mathbb{P}_{\operatorname{Bin}\left(n, R_{n}^{0}\right)}\left(D^{*}\right) \geq 1-n^{-\gamma}$, for all $n \geq N_{2}(\gamma)$ by Lemma 6.13. 
Case 3: The distance from $u$ to a side of $R_{n}^{0}$ is smaller than $n^{1 / 7}$ and from the other (only possible) side is smaller than $n^{1 / 5}$. In this case we will be working with $\mathbb{P}_{\left.P o\right|_{\mathbb{Q}}}$. Let

$$
J=\left\{j \in \mathbb{N}: n^{1 / 5} \leq 7^{j+1} \leq \beta n^{1 / 4}\right\}
$$

and consider the collection of partial annulus (two of the rectangles that composes the annulus will be out of $R) \mathcal{C}=\left\{\mathcal{A}_{j}: j \in J\right\}$. This case is a little more tricky. As the reader may have noticed, we didn't prove a result a result analogous to Theorems 6.5 and 6.10 for the quarter-plane. But, what happens is that since each one of the two rectangles that compose the annulus, are far from the boundary that the other is close. Note that:

$$
\begin{aligned}
& \mathbb{P}_{P o \mid \mathbb{Q}}\left(\mathbb{P}\left(O_{j} \mid \eta\right) \leq 2^{-4 k}\right) \leq \mathbb{P}_{\text {Po| }}\left(\mathbb{P}\left(B_{1} \mid \eta\right) \mathbb{P}\left(B_{2} \mid \eta\right) \leq 2^{-4 k}\right) \\
& \leq \mathbb{P}_{\text {Po|⿺⿻一⿰冫⿰亅⿱丿丶丶 }}\left(\bigcup_{i=1}^{2}\left(\mathbb{P}\left(B_{i} \mid \eta\right) \leq 2^{-4 k}\right)\right) \\
& \leq \sum_{i=1}^{2} \mathbb{P}_{P o \mid \mathbb{Q}}\left(\mathbb{P}\left(B_{i} \mid \eta\right) \leq 2^{-4 k}\right),
\end{aligned}
$$

where $B_{1}$ and $B_{2}$ represent the $3: 1$ rectangles that composes the annulus. Since both of them are at least $n^{1 / 10}$ far from the other boundary we may easily conclude that

$$
\mathbb{P}_{\text {Po| }}\left(\mathbb{P}\left(B_{i} \mid \eta\right) \leq 2^{-4 k}\right) \leq 2 \mathbb{P}_{\text {Po| } \mathbb{H}}\left(\mathbb{P}\left(B_{i} \mid \eta\right) \leq 2^{-4 k}\right)
$$

The rest of the proof will carry out in a similar way to Case 2. The only remaining detail is that now we have $\log (\beta)+\log (n) / 20$ annulus ( pick $N_{2}$ big enough, such that $\log (n) / 100 \geq|\log (\beta)|)$. So $N_{2}$ will depend both on $\gamma$ and $\beta$ ). We also note that this is only a problem when $\beta<1$. This will be the case when $\rho$, the aspect ratio of $R^{0}$ is bigger than 1 . The reader may check the remaining details.

\section{4}

\section{Variance and Influence}

While in the previous section the authors used a standard argument (bounding 1-arm events via the annulus method), in this one, they used a different strategy to bound the dependence of the crossing event on the set point $\eta$, where $\eta$ is taken accordingly to the binomial distribution, in terms of the expected influence of the colors. Remember that in this cases $\eta$ is a set 
of $n$ points in $R$, where each point is chosen independently and uniformly at random. Thus, it is natural to define $f_{R}^{\eta}:\{-1,1\}^{\eta} \rightarrow\{0,1\}$ for the Boolean function that encodes whether or not there exists a horizontal red crossing of $R$. Getting inspired by a the well-known inequality of Efron and Stein [5] they obtained the following theorem:

Theorem 6.16 For every fixed rectangle $R^{0} \subset \mathbb{R}^{2}$,

$$
\operatorname{Var}\left(\mathbb{P}\left(H_{R^{0}} \mid \eta\right)\right) \leq \sum_{i=1}^{n} \mathbb{E}\left[I_{m}\left(f_{R^{0}}^{\eta}\right)^{2}\right]
$$

where the the Variance and the Expectation are taken accordingly to the binomial distribution. Since we only use the binomial distribution until the end of this section, we will omit it to save up notation. The following corollary is an immediate consequence of the above result and Chebyshev's Inequality (A.6).

Corollary 6.17 Let $a(n)=\mathbb{E}\left[\sum_{m=1}^{n} I_{m}\left(f_{R^{0}}^{\eta}\right)^{2}\right]$. Then

$$
\mathbb{P}\left(\left|\mathbb{P}\left(H_{R^{0}} \mid \eta\right)-\mathbb{P}\left(H_{R^{0}}\right)\right| \geq a(n)^{1 / 3}\right) \leq a(n)^{1 / 3}
$$

Even though this is an original and non-trivial result, we will not prove it. In fact, we will prove a result in Chapter 7 that uses similar ideas to this one. Since, we don't want to be repetitive we will only present, we will only present our version, since it is an original and still unpublished result.

\section{5}

\section{Noise Sensitivity of crossings in Quenched Voronoi Percolation}

In this final section we will use the Revealment Theorem to obtain Theorem 6.2. We will also note that the proof that crossings are Noise Sensitive come as a corollary of this theorem. We remind that we are still working only with the binomial. So, let $R^{0}$ be a fixed rectangle with bounded aspect ratio $\rho \in(\theta, 1 / \theta)$. Since we aim to use the Revealment Theorem, we will define an algorithm with small revealment. The algorithm is essentially the same as that done in Chapter 5, so we shall describe it in a more intuitive way. We, again, refer to [15] for more details, since we also did this in a slightly informal way in Chapter 5.

Definição 6.18 (The Schramm-Steif randomized algorithm) Let $\mathcal{A}$ be the algorithm that, given $\eta$, queries bits of $\omega$ as follows: 
- Choose a point $x$ in the middle third of the left-hand side of $R^{0}$ uniformly at random.

- Explored the boundary between red and blue cells, with red on the left, starting from $x$. Here we place boundary conditions as follows: the lefthand side of $R^{0}$ is red above $x$, and blue below, and the bottom of $R^{0}$ is also blue. If this path:

- Reaches the right-hand side of $R^{0}$, then $f_{R^{0}}^{\eta}(\omega)=1$.

- Reaches the bottom of $R^{0}$, and ends at the top, then $f_{R^{0}}^{\eta}(\omega)=0$.

- Ends at the top of $R^{0}$ without reaching the bottom, then go to step 3 .

- Explore the boundary between red and blue cells, with red on the right, starting from $x$. Here we reverse the boundary conditions, i.e., the lefthand side of $R^{0}$ is blue above $x$, and red below, and the top of $R^{0}$ is also blue. If this path:

- Reaches the right-hand side of $R^{0}$, then $f_{R^{0}}^{\eta}(\omega)=1$.

- Otherwise $f_{R^{0}}^{\eta}(\omega)=0$.

Note that we only query those vertices whose cell we meet along one of our paths. Note that we can bound the revealment with the following lemma:

Lemma 6.19 Let $\mathcal{A}$ be the Schramm-Steif randomized algorithm. Then

$$
\delta_{\mathcal{A}}\left(f_{R^{0}}^{\eta}\right) \leq \max _{u \in \eta} \mathbb{P}\left(V\left(u, n^{-1 / 4}\right) \mid \eta\right)+O\left(n^{-1 / 4}\right)
$$

almost surely.

Proof. Let $u \in \eta$, and consider the probability that $u$ is queried by $\mathcal{A}$. First, note that the probability that the random start-point $x$ is within distance $n^{-1 / 4}$ of $u$ is $O\left(n^{-1 / 4}\right)$. But if the distance between $u$ and $x$ is greater than $n^{-1 / 4}$, and $u$ is queried by $\mathcal{A}$, then we have a red path to distance at least $n^{-1 / 4}$. So $V\left(u, n^{-1 / 4}\right)$ holds.

Now, we will use Proposition 6.15 to bound the probability of $V\left(u, n^{-1 / 4}\right)$.

Lemma 6.20 For every $\gamma$, there exists $c(\gamma)$ such that

$$
\mathbb{P}\left(\max _{u \in \eta} \mathbb{P}\left(V\left(u, n^{-1 / 4}\right) \mid \eta\right) \geq n^{-c}\right) \leq \frac{1}{n^{\gamma}}
$$

for every $n \geq N_{3}(\gamma, \theta)$. 
Proof. Let $R^{0}$ be a fixed rectangle of aspect ratio $\rho$. Renormalizing $R^{0}$ to have area $n$ would multiply its distances by $\sqrt{\frac{n}{\rho}}$. So, for every $\gamma>0$, we have by Proposition 6.15 and the union bound that there exists $c(\gamma+1)$ and $N_{3}(\gamma+1,1 / \sqrt{\rho})$, such that

$$
\begin{aligned}
\mathbb{P}\left(\max _{u \in \eta} \mathbb{P}\left(V\left(u, n^{-1 / 4}\right) \mid \eta\right) \geq n^{-c}\right) & \leq \sum_{i=1}^{n} \mathbb{P}\left(\mathbb{P}\left(V\left(u_{i}, \beta n^{1 / 4}\right) \mid \eta\right) \geq n^{-c}\right) \\
& \leq \frac{n}{n^{\gamma+1}}=n^{-\gamma}
\end{aligned}
$$

for every $n \geq N_{3}(\gamma+1,1 / \sqrt{\rho})$. Since $\rho \in(\theta, 1 / \theta)$, and $N$ will be bigger with smaller $1 / \sqrt{\rho}$ (bigger $\theta$ ), we actually have a dependence on a $N_{3}(\gamma, \theta)$.

Now, we will combine this with the following easy consequence of the Revealment Theorem for monotone functions (recall Proposition 5.8):

Theorem 6.21 Given a monotone function $f:\{-1,1\} \rightarrow\{0,1\}$ and a randomized algorithm $\mathcal{A}$ that determines $f$, we have that

$$
\sum_{m=1}^{n} I_{m}(f)^{2} \leq \delta_{\mathcal{A}}(f)
$$

From that and our previous bound we may conclude that

Lemma 6.22 Let $R^{0}$ be a rectangle with bounded aspect ratio $\rho \in(\theta, 1 / \theta)$. For every $\gamma>0$, there exists $c(\gamma)$ such that

$$
\mathbb{P}\left(\sum_{m=1}^{n} I_{m}\left(f_{R^{0}}^{\eta}\right)^{2} \geq n^{-c}\right) \leq \frac{1}{n^{\gamma}}
$$

for all $n \geq N(\gamma, \theta)$.

Note that with this Lemma and the BKS theorem we deduce that crossings in Quenched Voronoi Percolation are Noise Sensitive in the sense that

$$
\mathbb{E}\left[f_{R^{0}}^{\eta}(\omega) f_{R^{0}}^{\eta}\left(\omega_{\varepsilon}\right) \mid \eta\right]-\mathbb{E}\left[_{R^{0}}^{\eta} \mid \eta\right]^{2} \rightarrow 0
$$

as $n \rightarrow \infty$ with probability for every $\varepsilon \in(0,1)$. This is a natural definition since now $\mathbb{E}$ is also random.

We can now finally deduce Theorem 6.2.

Proof.[Proof of Theorem 6.2] Let $R^{0}$ be a rectangle with bounded aspect ratio $\rho \in(\theta, 1 / \theta)$. By Corollary 6.17 and the previous Lemma applied with $\gamma=2$ 
we have that there exists $c_{0}>0$ such that

$$
\mathbb{P}\left(\left|\mathbb{P}\left(H_{R^{0}} \mid \eta\right)-\mathbb{P}\left(H_{R^{0}}\right)\right| \geq n^{-c_{0}}\right) \leq n^{-c_{0}}
$$

for all $n \geq N(\theta)$, as required.

Before we end this Chapter we will show one more corollary of the results above. But for that, we will need a RSW version for the Binomial model. But, this is not so difficult to do, since we already have the RSW Theorem for annealed Voronoi Percolation, and AVP restricted to the half-plane. We exceptionally index the probability to avoid confusion, as we did before.

Theorem 6.23 (RSW Theorem for the Binomial Model) Let $R^{0}$ be a rectangle with aspect ratio $\rho>0$. Then, there exists $c_{2}(\rho)>0$ such that

$$
c_{2}<\mathbb{P}_{B i n\left(n, R^{0}\right)}\left(H_{R^{0}}\right)<1-c_{2}
$$

To prove that we will need two Lemmas which mimic Lemma 6.12 and Lemma 6.13, but for the opposite bound (Lemma 6.14 won't be needed in this case). We will only prove the analogous result to Lemma 6.12 since, as in the previous case, the proof regarding the $\left.\mathbb{P}_{\text {Po }}\right|_{\mathbb{H}}$ will be the same.

Lemma 6.24 Let $R_{\alpha n}^{\prime}$ be an axis-parallel rectangle strictly contained in the interior of $R_{n}$, where $0<\alpha \leq \frac{1}{4}$. Let $E \in \mathcal{F}_{R_{\alpha n}^{\prime}}$. Consider $R_{\alpha . n}^{\prime}$. If $\mathbb{P}_{\text {Po }}(E) \geq c$, then

$$
\mathbb{P}_{\operatorname{Bin}\left(n, R_{n}\right)}(E) \geq \frac{c}{4} e^{-2 / c},
$$

provided that $n \geq 64 c^{-1}$.

Proof. First, observe that in the proof of Lemma 6.12 we have proved that

$$
\mathbb{P}_{P o}(E)=\sum_{m \geq 0} \mathbb{P}_{P o \mid R_{n}}\left(E|| \eta \cap R_{\alpha n}^{\prime} \mid=m\right) \mathbb{P}_{\left.P o\right|_{R_{n}}}\left(\left|\eta \cap R_{\alpha n}^{\prime}\right|=m\right)
$$

and

$$
\mathbb{P}_{B i n\left(n, R_{n}\right)}(E)=\sum_{m \geq 0} \mathbb{P}_{\left.P o\right|_{R_{n}}}\left(E|| \eta \cap R_{\alpha n}^{\prime} \mid=m\right) \mathbb{P}_{B i n\left(n, R_{n}\right)}\left(\left|\eta \cap R_{\alpha n}^{\prime}\right|=m\right) .
$$

Note that by the properties of the Poisson distribution and Chebyshev's Inequality, we have that

$$
\mathbb{P}_{P o}\left(|| \eta \cap R_{\alpha n}^{\prime}|-\alpha n| \geq k \sqrt{n}\right) \leq \frac{\alpha \cdot n}{k^{2} n}=\frac{\alpha}{k^{2}},
$$

since $\mathbb{E}_{P o}\left[\left|\eta \cap R_{\alpha n}^{\prime}\right|\right]=\operatorname{Var}_{P o}\left[\left|\eta \cap R_{\alpha n}^{\prime}\right|\right]=\alpha . n$. 
Now, pick $k(c)=c^{-1 / 2}$. Observe that

$$
\begin{aligned}
c & \leq \sum_{m \geq 0} \mathbb{P}_{\left.P o\right|_{R_{n}}}\left(E|| \eta \cap R_{\alpha n}^{\prime} \mid=m\right) \mathbb{P}_{\left.P o\right|_{R_{n}}}\left(\left|\eta \cap R_{\alpha n}^{\prime}\right|=m\right) \\
& <\sum_{|m-n| \leq \alpha n} \mathbb{P}_{\left.P o\right|_{R_{n}}}\left(E|| \eta \cap R_{\alpha n}^{\prime} \mid=m\right) \mathbb{P}_{\left.P o\right|_{R_{n}}}\left(\left|\eta \cap R_{\alpha n}^{\prime}\right|=m\right)+\frac{c}{2}
\end{aligned}
$$

and we can conclude that

$$
\frac{c}{2}<\sum_{|m-n| \leq \alpha n} \mathbb{P}_{\left.P o\right|_{R_{n}}}\left(E|| \eta \cap R_{\alpha n}^{\prime} \mid=m\right) \mathbb{P}_{\left.P o\right|_{R_{n}}}\left(\left|\eta \cap R_{\alpha n}^{\prime}\right|=m\right)
$$

Now, similarly to what we have done, we will find a lower bound to the ratio $\sigma_{m} / x_{m}$. In this case, we claim that

Claim: Given that $n \geq 64 c^{-1}$, we have that for all $\alpha n-k \sqrt{n} \leq m \leq$ $\alpha n+k \sqrt{n}$,

$$
\frac{\sigma_{m}}{x_{m}} \geq \frac{e^{-2 / c}}{4}
$$

Proof. By similar approximations (using Stirling's formula), we have that for all $m$ in the desired interval

$$
\frac{\sigma_{m}}{x_{m}} \geq \frac{2 \pi}{e^{2}} \frac{(1-\alpha)^{n-m} e^{-m}}{e^{-\alpha n}}\left(\frac{n}{n-m}\right)^{n-m+1 / 2} .
$$

Since $k \sqrt{n}<n / 8$, by the imposed condition, we may also continuously extend this function. Again consider $m=\beta n$ for some $\beta \in\left[\alpha-\frac{k}{\sqrt{n}}, \alpha+\frac{k}{\sqrt{n}}\right]$ and define $h:\left[\alpha-\frac{k}{\sqrt{n}}, \alpha+\frac{k}{\sqrt{n}}\right] \rightarrow R^{+}$as:

$$
\begin{aligned}
h(\beta) & :=\frac{2 \pi}{e^{2} \sqrt{1-\beta}}\left(1-\frac{\alpha-\beta}{1-\beta}\right)^{(1-\beta) n} e^{(\alpha-\beta) n} \\
& \geq \frac{2 \pi}{e^{2} \sqrt{1-\beta}} e^{-\frac{(\alpha-\beta)^{2}}{1-\beta} n} \geq \frac{2 \pi}{e^{2}} e^{-2 k^{2}} \\
& \geq \frac{e^{-2 / c}}{4}
\end{aligned}
$$

With that, we may finally conclude that

$$
\begin{aligned}
\mathbb{P}_{B i n\left(n, R_{n}\right)}(E) & \geq \frac{e^{-c / 2}}{4} \sum_{|m-n| \leq \alpha n} \mathbb{P}_{P o \mid R_{n}}\left(E|| \eta \cap R_{n}^{\prime} \mid=m\right) \mathbb{P}_{\left.P o\right|_{n}}\left(\left|\eta \cap R_{n}^{\prime}\right|=m\right) \\
& >c \frac{e^{-c / 2}}{4}
\end{aligned}
$$


and so we conclude this result.

And a similar result will carry out for $\left.\mathbb{P}_{P o}\right|_{\mathbb{H}}$

Lemma 6.25 (Close to one boundary events.) Let $R_{\alpha n}^{\prime}$ be an axisparallel rectangle that has one of its sides "glued" to one of $R_{n}$ sides, where $0<\alpha \leq \frac{1}{4}$. Let $E \in \mathcal{F}_{R_{\alpha n}^{\prime}}$. Consider $R_{\alpha . n}^{\prime}$. If $\mathbb{P}_{P o \mid \mathbb{H}}(E) \geq c$, then

$$
\mathbb{P}_{\operatorname{Bin}\left(n, R_{n}\right)}(E) \geq \frac{c}{4} e^{-2 / c},
$$

provided that $n \geq 64 c^{-1}$.

Now we can prove the RSW-type result:

Proof.[Proof of RSW Theorem for the Binomial model] As we have remarked (many times) during this thesis, we only need to prove that for all $n \in \mathbb{N}$, $\mathbb{P}_{B i n\left(n, R^{0}\right)}\left(H_{R^{0}}\right) \geq c_{2}>0$ for $R^{0}$ with aspect ratio 1.5 , since we also have by duality that for all $n \in \mathbb{N}, \mathbb{P}_{\operatorname{Bin}(n, S)}\left(H_{S}\right)=1 / 2$, where $S$ is the square.

So consider $R^{0}$ a rectangle with aspect ratio $\rho=\frac{3}{2}$ and renormalize it to have area $n$. So, we can say without loss of generality that $R_{n}^{0}=[0, \sqrt{\rho \cdot n}] \times\left[0, \sqrt{\frac{n}{\rho}}\right]$. Now consider consider the two rectangles $R_{n}^{1}:=$ $\left[0, \frac{2}{3} \sqrt{\rho \cdot n}\right] \times\left[\frac{3}{8} \sqrt{\frac{n}{\rho}}, \frac{5}{8} \sqrt{\frac{n}{\rho}}\right]$ and $R_{n}^{2}:=\left[\frac{1}{3} \sqrt{\rho \cdot n}, \sqrt{\rho \cdot n}\right] \times\left[\frac{3}{8} \sqrt{\frac{n}{\rho}}, \frac{5}{8} \sqrt{\frac{n}{\rho}}\right]$. Also define $R_{n}^{3}$ to be the rectangle formed by the intersection between $R_{n}^{1}$ and $R_{n}^{2}$ and note that $R^{3}=\left[\frac{1}{3} \sqrt{\rho \cdot n}, \frac{2}{3} \sqrt{\rho \cdot n}\right] \times\left[\frac{3}{8} \sqrt{\frac{n}{\rho}}, \frac{5}{8} \sqrt{\frac{n}{\rho}}\right]$. Now, let $D_{j}, j \in\{1,2,3\}$ denote the event that for every $z \in R^{j}$, there exists some point $x \in \eta$ ( $\eta$ chosen accordingly to the Poisson distribution) at distance at most $n^{\frac{1}{32}}$ from $z$. Now, define the events $B_{1}:=H_{R_{n}^{1}} \cap D_{1}, B_{2}:=H_{R_{n}^{2}} \cap D_{2}$ and $B_{3}:=V_{R_{n}^{3}} \cap D_{3}$. Now, thanks to Theorem 4.5, Theorem 6.11, and the bound that we made on $D_{j}$, we have that there exists $c>0$, such that for all $n \geq N$,

$$
\mathbb{P}_{P o \mid \mathbb{H}}\left(B_{1}\right) \geq c, \mathbb{P}_{\text {Po| }}\left(B_{2}\right) \geq c \text { and } \mathbb{P}_{\text {Po }}\left(B_{3}\right) \geq c \text {. }
$$

Thanks to the conditions imposed by $D_{j}$, we have that $\left.B_{1}\right|_{\mathbb{H}} \in \mathcal{F}_{R_{1}^{\prime}},\left.B_{2}\right|_{\mathbb{H}} \in$ $\mathcal{F}_{R_{2}^{\prime}}$, and $B_{3} \in \mathcal{F}_{R_{3}^{\prime}}$, where each $R_{i}^{\prime}$ obey the hypothesis on Lemmas 6.24 and 6.25 (we won't give many details on this since we have done it before! Check it!). So, we have that there exists $\phi(c)$ and $N_{1}(c)$ (so they depend on the aspect ratio $\rho$ ) such that for all $n \geq N_{1}(c)$

$$
\mathbb{P}_{\operatorname{Bin}\left(n, R^{0}\right)}\left(B_{1}\right)=\mathbb{P}_{\operatorname{Bin}\left(n, R_{n}^{0}\right)}\left(B_{1}\right) \geq \phi, \mathbb{P}_{\operatorname{Bin}\left(n, R^{0}\right)}\left(B_{2}\right) \geq \phi \text { and } \quad P_{\operatorname{Bin}\left(n, R^{0}\right)}\left(B_{3}\right) \geq \phi
$$


Finally, by the FKG Inequality (see the image below), we conclude that for every $n$ (when $n<\max \left\{N, N_{1}\right\}$ this is trivial)

$$
\mathbb{P}_{\operatorname{Bin}\left(n, R^{0}\right)}\left(H_{R^{0}}\right) \geq c_{2}>0,
$$

and the rest of the RSW Theorem follows from that (by using well-known arguments at this point).

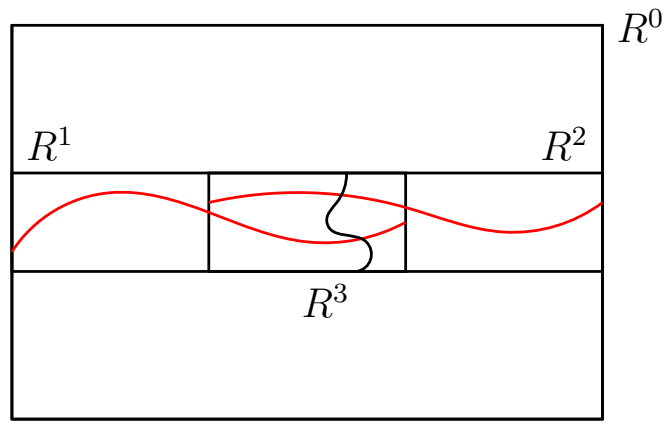

Figure 6.4: Note that the intersection of those three crossings make a horizontal red crossing in $R^{0}$.

So, we end this Chapter by observing that Theorem 6.2 with this RSW result gives us that

Theorem 6.26 For every $R$ with aspect ratio $\rho$, there exists $c(\rho)>0$ and $N(\rho) \in \mathbb{N}$, such that

$$
\mathbb{P}_{\operatorname{Bin}(n, R)}\left(c<\mathbb{P}\left(H_{R} \mid \eta\right)<1-c\right) \geq n^{-c},
$$

for all $n \geq N$. 


\section{7}

\section{On the rate of convergence in Quenched Voronoi Percolation}

In this final section we show that the arguments discussed in Chapter 6 may be iterated to obtain the following stronger bound on the probability that $\mathbb{P}\left(H_{R^{0}} \mid \eta\right)$ is far from its mean. We will denote by $\theta$ and $\tau$ two real constants in $(0,1)$.

Theorem 7.1 There exists a sequence $\gamma_{k}$ of positive real numbers such that the following holds: Let $R^{0}$ be an axis-parallel rectangle with bounded aspect ratio $\rho \in(\theta, 1 / \theta)$. For each $k \geq 2$ and for every $t \in[\tau, \infty)$ there exists $N^{(k)}(\tau, \theta) \in \mathbb{N}$ such that

$$
\mathbb{P}_{\operatorname{Bin}\left(n, R^{0}\right)}\left(\left|\mathbb{P}\left(H_{R^{0}} \mid \eta\right)-\mathbb{P}_{\operatorname{Bin}\left(n, R^{0}\right)}\left(H_{R^{0}}\right)\right| \geq t\right) \leq e^{-t \gamma_{k}(\log n)^{k}}
$$

for all $n \geq N^{(k)}$.

The structure of the proof will follow the same as in Theorem 6.2. Remind that by the end of Chapter 6 we got that

$$
\mathbb{P}_{B i n\left(n, R^{0}\right)}\left(\left|\mathbb{P}\left(H_{R^{0}} \mid \eta\right)-\mathbb{P}_{B i n\left(n, R^{0}\right)}\left(H_{R^{0}}\right)\right| \geq n^{-c}\right) \leq n^{-c},
$$

for all $n \geq N$. The natural idea is to pick such improved version and use it in place of Theorem 6.5 to get a stronger bound for the one-arm event. We would, then, turn ourselves back to Section 6.4 and use the Revealment Theorem to finally obtain that

$$
\mathbb{P}_{\operatorname{Bin}\left(n, R^{0}\right)}\left(\left|\mathbb{P}\left(H_{R^{0}} \mid \eta\right)-\mathbb{P}_{\operatorname{Bin}\left(n, R^{0}\right)}\left(H_{R^{0}}\right)\right| \geq t\right) \leq e^{-t \gamma_{2}(\log n)^{2}}
$$

for all $n \geq N^{(2)}$.

But this is, indeed, a very optimistic idea. When trying to do it in detail two problems arise. The first one is that we need the independence properties of the $\mathbb{P}_{\text {Po }}$ and $\mathbb{P}_{P \text { oo| } \mathbb{H}}$ models, but we have only proved (1) for the Binomial model. The other one is that Corollary 6.17 of Section 6.4 doesn't give us that the desired stronger outer bound of decay $e^{-c(\log n)^{2}}$. 
When trying to solve the first problem, it would be sufficient to prove a converse to Lemma 6.12, i.e., low probability events on the Binomial model implies that the same event has low probability in the Poisson model. Unfortunately, this is impossible (consider the event that the event that the number of points in $R$ is not $n$, for example). So, we will study crossings in a smaller rectangle $R$, inside $4 R$. This can be understood as a different model, but we will simply think as the study of the event $H_{R}$ for the rectangle $4 R$, following the notation $\mathbb{P}_{\operatorname{Bin}(n, 4 R)\left(H_{R}\right)}$.

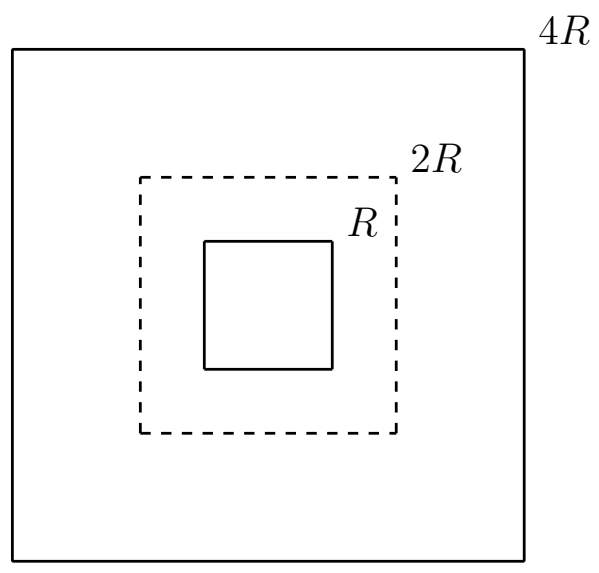

Figure 7.1: When we study such model one may note that we are studying events that depend on points that are far enough from the boundary This makes possible the comparison between this model and the Poisson one as we will see later. We also draw in dashed lines the rectangle $2 R$. It will be useful to think of him since we will be studying events $E \in \mathcal{F}_{2 R}$ because the event $H_{R}$ will depend solely on points inside $2 R$ with a very high probability.

Note that this model urges to be compared with the Poisson Model. In fact, we have already established one side of the comparison with Lemma 6.12. The other side will be done in the next Section, see Lemma 7.5.

Our strategy to resolve the first problem is now clear. We simply prove (1) for the model $\mathbb{P}_{\operatorname{Bin}(n, 4 R)}$ and deduce an equivalent bound in the Poisson model using Lemma 7.5. More generally, as we can translate back and forth between the $\mathbb{P}_{P o}$ and $\mathbb{P}_{\operatorname{Bin}(n, 4 R)}$ models more easily, it is natural to prove the main result first for the $\mathbb{P}_{\operatorname{Bin}(n, 4 R)}$ model.

Theorem 7.2 There exists a sequence $\gamma_{k}$ of positive real numbers such that the following holds: Let $R^{0}$ be an axis-parallel rectangle with bounded aspect ratio $\rho \in(\theta, 1 / \theta)$. For each $k \geq 2$ and for every $t \in[\tau, \infty)$ there exists $N^{(k)}(\tau, \theta) \in \mathbb{N}$ such that

$$
\mathbb{P}_{\operatorname{Bin}\left(n, 4 R^{0}\right)}\left(\left|\mathbb{P}\left(H_{R^{0}} \mid \eta\right)-P_{\operatorname{Bin}\left(n, 4 R^{0}\right)}\left(H_{R^{0}}\right)\right| \geq t\right) \leq e^{-t \gamma_{k}(\log n)^{k}}
$$


for all $n \geq N^{(k)}$.

To deal with boundaries, we will also need to do the same for $\mathbb{P}_{\left.P o\right|_{\mathbb{H}}}$. It is useful to define $2 R^{*}$ and $4 R^{*}$ as translations of $2 R$ and $4 R$. Specifically, translated so that they are contained in the half plane. So we will study $H_{R}$ inside the rectangle $4 R^{*}$.

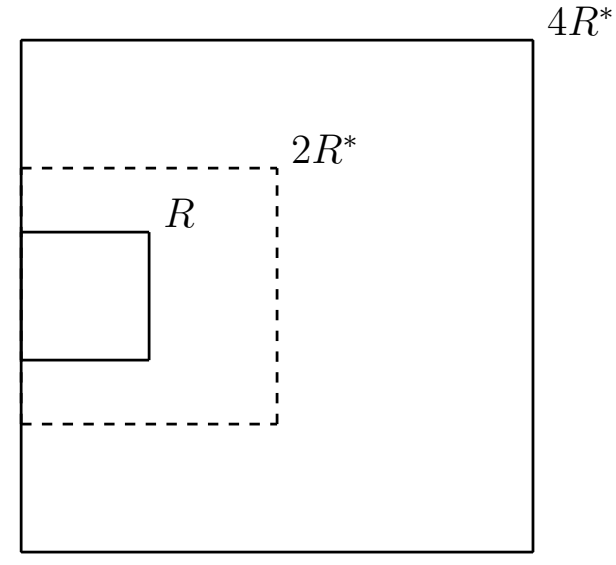

Figure 7.2: Now we will study crossings of a rectangle glued to one boundary and far from the others. This makes possible the comparison between this model and the Poisson restricted to the half plane, where the half plane is positioned in the same glued side. We also draw in dashed lines the rectangle $2 R^{*}$. It will be useful to think of him since we will be studying events $E \in \mathcal{F}_{2 R^{*}}$ (when naturally restricted to $\mathbb{H}$ ).

We remark that we also need to do the same thing for $R$ glued to the top, bottom and right side. Since all the results are naturally valid for all those cases we will omit doing it to avoid being repetitive. This model is naturally compared to $\mathbb{P}_{P o \mid \mathbb{H}}$ where we take $\mathbb{H}$ to coincide with left side of $R$.

We now turn to the second problem. In the previous Chapter we used Corollary 6.17, which states that

$$
\mathbb{P}\left(\left|\mathbb{P}\left(H_{R^{0}} \mid \eta\right)-\mathbb{P}\left(H_{R^{0}}\right)\right| \geq a(n)^{1 / 3}\right) \leq a(n)^{1 / 3}
$$

where $a(n)=\mathbb{E}\left[\sum_{m=1}^{n} I_{m}\left(f_{R^{0}}^{\eta}\right)^{2}\right]$, to achieve our bound (1). In order to achieve a stronger probability bound (at the cost of considering a larger deviation) we require a different inequality relating $\left|\mathbb{P}\left(H_{R^{0}} \mid \eta\right)-\mathbb{P}\left(H_{R^{0}}\right)\right|$ with $a(n)$. This inequality is given in Section 7.3, see Theorem 7.14.

So, to give an overview of this chapter, we will divide it in 5 sections. The first section will study the relations between all the defined models. In the second section we give improved bounds on one-arm events and use them, together with the Revealment Theorem, to give improved bounds on the sum of the squares of influences. The third section will be the stronger version 
of Corollary 6.17 following a similar proof to that given in [1]. In the fourth section we will finally kick-start the induction and prove Theorem 7.2. In the fifth section we return to the original model and deduce Theorem 7.1.

\section{1}

\section{Relations between the models}

In this section we will establish the connection between the models. Since $2 R$ has one fourth of the area of $4 R$, we can obtain from what was done in Section 6.3 that:

Lemma 7.3 (Far from the boundary events) Let $k=n / 16$. Consider $E \in \mathcal{F}_{2 R_{k}}$. Then

$$
\mathbb{P}_{\text {Bin }\left(n, 4 R_{k}\right)}(E) \leq 3 \mathbb{P}_{\text {Po }}(E)
$$

Lemma 7.4 (Close to one boundary events) Let $k=n / 16$ and $E \in$ $\mathcal{F}_{2 R_{k}^{*}}$. Then

$$
\mathbb{P}_{\operatorname{Bin}\left(n, 4 R_{k}^{*}\right)}(E)<3 \mathbb{P}_{\text {Po| } \mathbb{H}}(E) .
$$

But, as we have previously remarked, we also want those Lemmas in the "opposite" direction.

Lemma 7.5 Let $E \in \mathcal{F}_{2 R}$. If there exists $\varepsilon>0$ such that for every $m \geq N_{1}$ :

$$
\mathbb{P}_{\operatorname{Bin}(m, 4 R)}(E)<\varepsilon,
$$

then, we have that

$$
\mathbb{P}_{\text {Po }}(E)<\varepsilon+\mathbb{P}_{\text {Po }}\left(|\eta \cap 4 R|<N_{1}\right) .
$$

Proof. Let $R, 2 R$ and $4 R$ be fixed. Since $E \in \mathcal{F}_{2 R}$ :

$$
\begin{aligned}
\mathbb{P}_{\text {Po }}(E) & =\sum_{m \geq 0} \mathbb{P}_{\text {Po }}(E|| \eta \cap 4 R \mid=m) \mathbb{P}_{\text {Po }}(|\eta \cap 4 R|=m) \\
& =\sum_{m \geq 0} \mathbb{P}_{\text {Po| } 4 R}(E|| \eta \cap 4 R \mid=m) \mathbb{P}_{\text {Po }}(|\eta \cap 4 R|=m) \\
& =\sum_{m \geq 0} \mathbb{P}_{\text {Bin }(m, 4 R)}(E) \mathbb{P}_{\text {Po }}(|\eta \cap 4 R|=m) \\
& \leq \varepsilon+\mathbb{P}_{\text {Po }}\left(|\eta \cap 4 R|<N_{1}\right) .
\end{aligned}
$$

As an easy consequence of the above lemma for $N_{1}=n$ we deduce the following corollary: 
Corollary 7.6 Let $R_{n}$ be a rectangle of area $n$. Let $E \in \mathcal{F}_{2 R_{n}}$. If there exists $\varepsilon>0$ such that for every $m \geq n$ :

$$
\mathbb{P}_{\operatorname{Bin}\left(m, 4 R_{n}\right)}(E)<\varepsilon
$$

Then

$$
\mathbb{P}_{\text {Po }}(E)<\varepsilon+e^{-n}
$$

Again, we may obtain the same result for the half-plane. We will state only on the latter form, since it will be more useful for our purposes.

Lemma 7.7 Let $R_{n}$ be a rectangle of area $n$. Let $E \in \mathcal{F}_{2 R_{n}^{*}}$. If there exists $\varepsilon>0$ such that for every $m \geq n$ :

$$
\mathbb{P}_{\operatorname{Bin}\left(m, 4 R_{n}^{*}\right)}(E)<\varepsilon
$$

Then

$$
\mathbb{P}_{P o \mid H}(E)<\varepsilon+e^{-n} \text {. }
$$

Now that we have built the desired connection, we may start generalizing the desired results.

\section{2}

\section{Improved bound for the sum of squares of the Influences}

In this section we will give an improved bound on the probability that the sum of squares of the Influences is at least $n^{-\varepsilon}$. This result (Theorem 7.8) is a strengthening of Lemma 6.22. Our approach, is similar to that given in Chapter 6 . The main difference is that we prove an improved bound for the one arm event. We also make some other minor changes, such as to the randomized algorithm.

We remark that we will first do this for the models $\mathbb{P}_{\text {Po }}$ and $\mathbb{P}_{\operatorname{Bin}(n, 4 R)}$. Doing it for the models $\mathbb{P}_{P o \mid \mathbb{H}}$ and $\mathbb{P}_{\operatorname{Bin}\left(n, 4 R^{*}\right)}$ will be essentially equivalent and we will just point out to the reader how to cover the remaining details.

For $\eta$, a set of $n$ points in $4 R$, each of them chosen uniformly and independently at random, we denote by $g_{R}^{\eta}:\{-1,1\}^{\eta} \rightarrow\{0,1\}$ the function which encodes whether or not there is a horizontal red crossing in $R$ in the Voronoi tiling given by $\eta$. Do not confuse this function with the function $f_{R}^{\eta}$ of Chapter 6 ! The difference is that the points are now distributed in $4 R$, while the function still encodes the existence of a red horizontal crossing in $R$. 
Theorem 7.8 Let $R^{1}$ be an axis-parallel rectangle of aspect ratio $3: 1$. Suppose that there exist constants $c, a \in \mathbb{R}^{+}$and $k, N \geq 1$ such that

$$
\mathbb{P}_{\text {Po }}\left(c<\mathbb{P}\left(H_{R_{n}^{1}} \mid \eta\right)<1-c\right) \geq 1-e^{-a(\log n)^{k}},
$$

for all $n \geq N$. Then, for every axis-parallel rectangle $R^{0}$ of bounded aspect ratio $\rho \in(\theta, 1 / \theta)$, there exist constants $M_{1}(a, \theta, k, N)$ and $\varepsilon(c)$ such that

$$
\mathbb{P}_{\operatorname{Bin}\left(n, 4 R^{0}\right)}\left(\sum_{\ell=1}^{n} I_{\ell}^{2}\left(g_{R^{0}}^{\eta}\right) \geq n^{-\varepsilon}\right) \leq e^{-\frac{a}{100}(\log n)^{k+1}}
$$

for all $n \geq M_{1}$.

To prove this Theorem, we will need the following Proposition that achieves an improved bound for the one-arm event. Again we focus on a onearm event to distance $\rho^{-1 / 2} n^{1 / 4}$.

Proposition 7.9 Suppose that condition (2) holds with constants $c, a, k, N$ and that $R^{0}$ is an axis-parallel rectangle with aspect ratio $\rho \in(\theta, 1 / \theta)$. Then, there exist constants $\varepsilon(c)$ and $N_{1}(a, \theta, k, N)$ such that the following holds. Let $u \in \frac{3}{2} R_{n}^{0}$ and define $\beta:=1 / \sqrt{\rho}$. Then

$$
\mathbb{P}_{\operatorname{Bin}\left(n, 4 R_{n}^{0}\right)}\left(\mathbb{P}\left(V\left(u, \beta n^{\frac{1}{4}} \mid \eta\right) \geq n^{-\varepsilon}\right)\right) \leq e^{-\frac{a}{80}(\log (n))^{k+1}}
$$

for every $n \geq N_{1}$.

Proof. Fix $u \in \frac{3}{2} R_{n}^{0}$. For each $j \in \mathbb{N}$, let $A_{j}$ denote the square annulus, centered on $u$, with inner side-length $7^{j}$ and outer side-length $3.7^{j}$. Let $O_{j}$ be the event that there exists a blue circuit around the annulus $A_{j}$. Let

$$
J=\left\{j \in \mathbb{N}: n^{\frac{1}{8}} \leq 7^{j+1} \leq \beta n^{\frac{1}{4}}\right\}
$$

Denote by $\mathcal{C}=\left\{A_{j}: j \in J\right\}$ the collection of annulus (which won't be partial in that case because of the way we set the distances). Pick a sufficiently big $l(c)>0$, so that $2^{-l}<c$, where $c$ is the same constant as in (2). Now, for each $j \in J$, let $D_{j}^{(1)}$ denote the event that $\mathbb{P}\left(O_{j} \mid \eta\right)>2^{-4 l}$ and let $D_{j}^{(2)}$ denote the event that for every $z \in A_{j}$, there exists some point $x \in \eta$ at distance at most $n^{\frac{1}{32}}$ from $z$. Finally, define

$$
D_{j}:=D_{j}^{(1)} \cap D_{j}^{(2)},
$$

and note that the events $D_{j}$ are independent with respect to the Poisson distribution, since the distance between two annulus is $4.7^{j}$, which is always bigger than the condition imposed by $D_{j}^{(2)}$. So, again, just as it happened in 
Chapter 6 we will have a collection of events which depend on disjoint sets, being independent with respect to $\mathbb{P}_{P o}$. Next we observe that

$$
\mathbb{P}_{\text {Po }}\left(D_{j}^{(2)}\right) \geq 1-e^{-n^{1 / 32}} \geq 1-e^{-a(\log n)^{k}}
$$

for all $n \geq N(a, k)$. Next, observe that, $A_{j}$ is composed of 4 rectangles with aspect ratio $3: 1$. Then, by the FKG inequality combined with condition (2) we get that

$$
\mathbb{P}_{\text {Po }}\left(\mathbb{P}\left(O_{j} \mid \eta\right) \leq 2^{-4 l}\right) \leq 4 e^{-a(\log n)^{k}}
$$

for all $n \geq N^{8}$. So, $\mathbb{P}_{P o}\left(D_{j}^{c}\right) \leq 5 e^{-a(\log n)^{k}}$, for $n \geq \max \left\{N(a, k), N^{8}\right\}$

Now consider $D^{*}$ the event that $D_{j}$ holds for at least half of the elements $j \in J$, and observe that there exists $N^{*}(a, \theta, k)$ such that

$$
\mathbb{P}_{\text {Po }}\left(D^{*}\right) \geq 1-2^{|J|}\left(5 e^{-a(\log n)^{k}}\right)^{|J| / 2} \geq 1-e^{-\frac{a}{64}(\log n)^{k+1}},
$$

for all $n \geq N^{*}(a, \theta)$ (in fact $N^{*}=e^{8 a^{-1}}+\theta^{-50}$ is sufficiently large).

We now observe that $D^{*} \in \mathcal{F}_{2 R_{n}^{0}}$. Consider now Lemma 7.3 to obtain that

$$
\mathbb{P}_{\operatorname{Bin}\left(n, 4 R_{n}^{0}\right)}\left(D^{*}\right) \geq 1-5 e^{-\frac{\alpha}{64}(\log (n))^{k+1}},
$$

for all $n \geq \max \left\{N(a, k), N^{*}(a, \theta), N^{8}\right\}$. So, there exists $N^{* *}(a)$ such that

$$
\mathbb{P}_{\operatorname{Bin}\left(n, 4 R_{n}^{0}\right)}\left(D^{*}\right) \geq 1-e^{-\frac{\alpha}{80}(\log (n))^{k+1}},
$$

for all $n \geq \max \left\{N(a, k), N^{*}(a, \theta), N^{* *}(a), N^{8}\right\}$.

Finally, for those $\eta$ such that $D^{*}$ holds, we get that

$$
\begin{aligned}
\mathbb{P}_{\operatorname{Bin}\left(n, 4 R_{n}^{0}\right)}\left(V\left(u, n^{\frac{1}{4}} \mid \eta\right)\right) \leq \mathbb{P}_{\operatorname{Bin}\left(n, 4 R_{n}^{0}\right)}\left(\bigcap_{j \in J} O_{j}^{c} \mid \eta\right) & =\prod_{j \in J} \mathbb{P}_{\operatorname{Bin}\left(n, 4 R_{n}^{0}\right)}\left(O_{j}^{c} \mid \eta\right) \\
& \leq\left(1-2^{-4 l}\right)^{|J| / 2} \leq n^{-\varepsilon}
\end{aligned}
$$

for some $\varepsilon(l)>0$. Pick $N_{1}=\max \left\{N^{8}, N(a, k), N^{*}(a, \theta), N^{* *}(a)\right\}$ and we get the desired result, since $l$ depends on $c$.

Consider the rectangle $R^{0}$. Now, just as we did in Chapter 6, we will define an algorithm $\mathcal{A}$ that decides $g_{R^{0}}^{\eta}$ and has low revealment. The algorithm will be, essentially the same as in Chapter 6 , but now, we want to decide a horizontal red crossing in $R^{0}$ instead of $4 R^{0}$. So, we will do the following:

Definição 7.10 (Slightly modified Schramm-Steif randomized algorithm)

Let $\mathcal{A}$ be the algorithm that, given $\eta$, queries bits of $\omega$ as follows. First, consider the restriction of $\eta$ to $R^{0},\left.\eta\right|_{R^{0}}$. With that, the algorithm will follow in the exact same way: 
- Choose a point $x$ in the middle third of the left-hand side of $R^{0}$ uniformly at random.

- Explored the boundary between red and blue cells, with red on the left, starting from $x$. Here we place boundary conditions as follows: the lefthand side of $R^{0}$ is red above $x$, and blue below, and the bottom of $R^{0}$ is also blue. If this path:

- Reaches the right-hand side of $R^{0}$, then $g_{R^{0}}^{\eta}(\omega)=1$.

- Reaches the bottom of $R^{0}$, and ends at the top, then $g_{R^{0}}^{\eta}(\omega)=0$.

- Ends at the top of $R^{0}$ without reaching the bottom, then go to step 3 .

- Explore the boundary between red and blue cells, with red on the right, starting from $x$. Here we reverse the boundary conditions, i.e., the lefthand side of $R^{0}$ is blue above $x$, and red below, and the top of $R^{0}$ is also blue. If this path:

- Reaches the right-hand side of $R$, then $g_{R^{0}}^{\eta}(\omega)=1$.

- Otherwise $g_{R^{0}}^{\eta}(\omega)=0$.

Note that this algorithm queries a bit outside $\frac{3}{2} R^{0}$ with probability $O\left(n^{-1 / 4}\right.$ ) (in fact, we much lower probability as we saw with the events $D_{j}^{(2)}$ ). So, we may derive a bound for the revealment with the same argument as we did in Lemma 6.19:

Lemma 7.11 Let $\mathcal{A}$ be the alternate Schramm-Steif randomized algorithm.

Then

$$
\delta_{\mathcal{A}}\left(g_{R^{0}}^{\eta}\right) \leq \max _{u \in \eta \cap \frac{3}{2} R^{0}} \mathbb{P}\left(V\left(u, n^{-1 / 4}\right) \mid \eta\right)+O\left(n^{-1 / 4}\right)
$$

almost surely.

Now, we may use Proposition 7.9 to conclude that

Lemma 7.12 We have that

$$
\mathbb{P}_{\operatorname{Bin}\left(n, 4 R^{0}\right)}\left(\mathbb{P}\left(V\left(u, n^{-\frac{1}{4}} \mid \eta\right) \geq n^{-\varepsilon}\right)\right) \leq e^{-\frac{a}{100}(\log (n))^{k+1}}
$$

for every $n \geq M_{1}(a, \theta, k, N)$.

Proof. When we re-normalize $R^{0}$ to have area $n$ the distances are multiplied by $\beta n^{1 / 2}$, where $\beta=1 / \sqrt{\rho}$. By the union bound and the fact that there are at most $n$ points in $\frac{3}{2} R_{n}^{0}$ we may conclude by Proposition 7.9 that

$$
\mathbb{P}_{B i n\left(n, 4 R^{0}\right)}\left(\mathbb{P}\left(V\left(u, n^{-\frac{1}{4}} \mid \eta\right) \geq n^{-\varepsilon}\right)\right) \leq n \cdot e^{-\frac{a}{80}(\log (n))^{k+1}}
$$


for every $n \geq N_{1}(a, \theta, k, N)$ (note that the worst cases for $\beta$ are the extremes $1 / \theta$ and $\theta)$. Since there exists $N^{* * *}(a)$ such that

$$
n e^{-\frac{a}{80}(\log n)^{k+1}} \leq e^{-\frac{a}{100}(\log n)^{k+1}}
$$

for all $n \geq N^{* * *}(a)$, we can pick $M_{1}=\max \left\{N_{1}, N^{* * *}(a)\right\}$, and get the desired result.

Proof.[Proof of Theorem 7.8] Finally, one may deduce with the Revealment Theorem for monotone functions that there exists $\varepsilon(c)$ such that

$$
\mathbb{P}_{\operatorname{Bin}\left(n, 4 R^{0}\right)}\left(\sum_{\ell=1}^{n} I_{\ell}^{2}\left(g_{R^{0}}^{\eta}\right) \geq n^{-\epsilon}\right) \leq e^{\frac{a}{100}(\log n)^{k+1}}
$$

for all $n \geq M_{1}(a, \theta, k, N):=\max \left\{N^{8}, N(a, k), N^{*}(a, \theta), N^{* *}(a), N^{* * *}(a)\right\}$.

Now, we want this to carry out to the models $\mathbb{P}_{P o \mid \mathbb{H}}$ and $\mathbb{P}_{B i n\left(n, 4 R^{0 *}\right)}$. For that, first, define $h_{R}^{\eta}$ to be the same as $g_{R}^{\eta}$, but the set of $n$ points $\eta$ is taken, naturally, in $4 R^{*}$.

Theorem 7.13 Let $R^{1}$ be an axis-parallel rectangle of aspect ratio $3: 1$. Suppose that there exists $c, a \in \mathbb{R}^{+}$and $k, N \in \mathbb{N}$ such that

$$
\mathbb{P}_{\text {Po| } \mathbb{H}}\left(c<\mathbb{P}\left(H_{R_{n}^{1}} \mid \eta\right)<1-c\right) \geq 1-e^{-a(\log n)^{k}},
$$

for all $n \geq N$. Then, for every axis-parallel rectangle $R^{0}$ of bounded aspect ratio $\rho \in(\theta, 1 / \theta)$, there exists $M_{1}(a, \theta, k, N)$ and $\varepsilon(c)$ such that

$$
\mathbb{P}_{\operatorname{Bin}\left(n, 4 R^{0 *}\right)}\left(\sum_{\ell=1}^{n} I_{\ell}^{2}\left(h_{R^{0}}^{\eta}\right) \geq n^{-\varepsilon}\right) \leq e^{-\frac{a}{100}(\log n)^{k+1}}
$$

for all $n \geq M_{1}$.

The only difference on the proof of this Theorem, will be the proof of Proposition 7.9. But, we don't even have to break in cases as we did in Chapter 6 , since we are comparing just with $\mathbb{P}_{\left.P o\right|_{\mathbb{H}}}$. The only difference is that some annulus will be partial (formed by three rectangles) and some will be full. Since we don't want to be over repetitive, we trust the reader to convince themselves of that and carry out the remaining details.

\section{3}

\section{Improved bound on the crossing probabilities for Voronoi Percolation}

This section will mimic the result of Section 6.4. We will be working with the model $\mathbb{P}_{B i n(n, 4 R)}$ (or $\mathbb{P}_{B i n\left(n, 4 R^{*}\right)}$ all the time. The idea will be that combined with Theorem 7.8 and Theorem 6.5 (or 6.10), we will be able to start and induction, and prove the desired improved bound. We will begin proving 
it for $\mathbb{P}_{\operatorname{Bin}(n, 4 R)}$, but the reader will note that it is exactly analogous to the case $\mathbb{P}_{\operatorname{Bin}\left(n, 4 R^{*}\right)}$.

Theorem 7.14 Let $R$ be an axis parallel rectangle and denote by $R^{\prime}$ its rotation by $\pi / 2$. Suppose that there exists $k \geq 2, M_{1} \in \mathbb{N}, \gamma>0$ and $\varepsilon>0$ such that

$$
\begin{gathered}
\mathbb{P}_{\operatorname{Bin}(n, 4 R)}\left(\sum_{\ell=1}^{n} I_{\ell}^{2}\left(g_{R}^{\eta}\right) \geq n^{-\varepsilon}\right) \leq e^{-\gamma(\log n)^{k}} \\
\mathbb{P}_{\operatorname{Bin}\left(n, 4 R^{\prime}\right)}\left(\sum_{\ell=1}^{n} I_{\ell}^{2}\left(g_{R^{\prime}}^{\eta}\right) \geq n^{-\varepsilon}\right) \leq e^{-\gamma(\log n)^{k}}
\end{gathered}
$$

for all $n \geq M_{1}$. Then, for any $t>0$, there exists $N_{2}(t, \gamma, \varepsilon, k)$ such that:

$$
\mathbb{P}_{\operatorname{Bin}(n, 4 R)}\left(\left|\mathbb{P}\left(H_{R} \mid \eta\right)-\mathbb{P}_{\operatorname{Bin}(n, 4 R)}\left(H_{R}\right)\right| \geq t\right) \leq e^{-t(\log n)^{k} \frac{\gamma}{100}}
$$

for all $n \geq N_{2}$.

For the proof of this Theorem, we require the following Lemma. Note how it is similar to the idea of relating Variance and Influence in Section 6.4:

Lemma 7.15 For all $\lambda>0$ we have

$$
\operatorname{Var}_{B i n(n, 4 R)}\left(e^{\frac{\lambda}{2} Z}\right) \leq \frac{\lambda^{2}}{4} \mathbb{E}_{B i n(n, 4 R)}\left[e^{\lambda Z} \sum_{\ell=1}^{n} e^{\lambda . I_{\ell}\left(g_{R}^{\eta}\right)} I_{\ell}^{2}\left(g_{R}^{\eta}\right)\right]
$$

where $Z:=\mathbb{P}\left(H_{R} \mid \eta\right)$.

Proof. Define $q^{\eta}:=e^{\lambda Z / 2}$ for a fixed $\lambda>0$ and $q_{m}:=\mathbb{E}_{\operatorname{Bin}(n, 4 R)}\left[q^{\eta} \mid \mathcal{F}_{m}\right]$ where $\mathcal{F}_{m}$ is the $\sigma$-algebra generated by $\eta_{1}, \ldots, \eta_{m}$. Define $\eta^{-}$as $\eta$ with $\eta_{m}$ deleted. We have by the Martingale properties that (see [1] for more details):

$$
\operatorname{Var}_{\operatorname{Bin}(n, 4 R)}\left(q_{m}-q_{m-1}\right) \leq \mathbb{E}_{\operatorname{Bin}(n, 4 R)}\left[\left(q^{\eta}-q^{\eta^{-}}\right)^{2}\right]
$$

Now we will prove the following claim that will complete the proof

Claim: We have that

$$
\left|q^{\eta}-q^{\eta^{-}}\right| \leq \frac{\lambda}{2} e^{\frac{\lambda}{2} Z} e^{\frac{\lambda}{2} \cdot I_{m}\left(g_{R}^{\eta}\right)} I_{m}\left(g_{R}^{\eta}\right), \text { almost surely }
$$

To see this, first consider the case $q^{\eta}>q^{\eta^{-}}$and observe that by the Mean Value Theorem

$$
q^{\eta}-q^{\eta^{-}} \leq \frac{\lambda}{2} e^{\frac{\lambda}{2} Z} I_{m}\left(g_{R}^{\eta}\right), \text { almost surely }
$$


When $q^{\eta}<q^{\eta^{-}}$, we combine the MVT with the fact that $\mid \mathbb{P}\left(H_{R} \mid \eta\right)$ $\mathbb{P}\left(H_{R} \mid \eta^{-}\right) \mid \leq I_{m}\left(g_{R}^{\eta}\right)$, almost surely (see [1] for more details; despite being a different model, it will follow from the same argument). So, we get that:

$$
q^{\eta^{-}}-q^{\eta} \leq \frac{\lambda}{2} e^{\frac{\lambda}{2} Z} e^{\frac{\lambda}{2} \cdot I_{m}\left(g_{R}^{\eta}\right)} I_{m}\left(g_{R}^{\eta}\right), \text { almost surely }
$$

proving the desired claim. Since

$$
\operatorname{Var}_{B i n(n, 4 R)}\left(q^{\eta}\right)=\sum_{m=1}^{n} \operatorname{Var}_{B i n(n, 4 R)}\left(q_{m}-q_{m-1}\right)
$$

we conclude the Lemma.

It is also useful to note the following lemma from analysis (see Lemma 3.19 of [5] for more details):

Lemma 7.16 Let $G:(0,1) \rightarrow(0,+\infty)$ be a function that satisfies the following properties:

1. $\lim _{x \rightarrow 0} \frac{(G(x)-1)}{x}=0$

2. $\left(1-x^{2}\right) G(x) \leq G(x / 2)^{2}$, for every $x \in(0,1)$.

Then;

$$
G(x) \leq\left(1-x^{2}\right)^{-2}
$$

for every $x \in(0,1)$.

We are now ready to prove the theorem.

Proof.[Proof of Theorem 7.14] Define $\mathcal{A}:=\left\{\eta: \sum_{\ell=1}^{n} I_{\ell}^{2}\left(g_{R}^{\eta}\right) \leq n^{-\varepsilon}\right\}$. Observe that when the event $\mathcal{A}$ holds, we have that for any $0<\lambda<n^{\varepsilon / 2}$

$$
\sum_{\ell=1}^{n} e^{\lambda . I_{\ell}\left(g_{R}^{\eta}\right)} I_{\ell}^{2}\left(g_{R}^{\eta}\right) \leq e . n^{-\varepsilon}
$$

So, by the Inequality (10), we have that for all $\lambda<n^{\epsilon / 2}$ :

$$
\begin{aligned}
\mathbb{E}_{\operatorname{Bin}(n, 4 R)}\left[e^{\lambda Z}\right]- & \mathbb{E}_{\operatorname{Bin}(n, 4 R)}^{2}\left[e^{\frac{\lambda}{2} Z}\right] \leq \frac{\lambda^{2}}{4} \mathbb{E}_{\operatorname{Bin}(n, 4 R)}\left[e^{\lambda Z} \sum_{\ell=1}^{n} e^{\lambda . I_{\ell}\left(g_{R}^{\eta}\right)} I_{\ell}^{2}\left(g_{R}^{\eta}\right)\right] \\
& \leq \frac{e \lambda^{2}}{4 n^{\varepsilon}} \mathbb{E}_{\operatorname{Bin}(n, 4 R)}\left[e^{\lambda Z}\right]+\frac{\lambda^{2}}{4} \mathbb{E}_{\operatorname{Bin}(n, 4 R)}\left[e^{\lambda Z} \sum_{\ell=1}^{n} e^{\lambda . I_{\ell}\left(g_{R}^{\eta}\right)} I_{\ell}^{2}\left(g_{R}^{\eta}\right) \mathbb{1}_{\mathcal{A}^{c}}\right]
\end{aligned}
$$

Observe that for the second part of the equation:

$$
\frac{\lambda^{2}}{4} \mathbb{E}_{\operatorname{Bin}(n, 4 R)}\left[e^{\lambda Z} \sum_{\ell=1}^{n} e^{\lambda \cdot I_{\ell}\left(g_{R}^{\eta}\right)} I_{\ell}^{2}\left(g_{R}^{\eta}\right) \mathbb{1}_{\mathcal{A}^{c}}\right] \leq n \frac{\lambda^{2} e^{2 \lambda}}{4} \mathbb{P}_{\operatorname{Bin}(n, 4 R)}\left(\mathcal{A}^{c}\right)
$$


Also, by Condition 8 of our statement, we have that for all $n \geq M_{1}$ :

$$
\mathbb{P}_{\operatorname{Bin}(n, 4 R)}\left(\mathcal{A}^{c}\right) \leq e^{-\gamma(\log n)^{k}}
$$

So, for $\lambda<\frac{\gamma}{3}(\log n)^{k}$

$$
\frac{\lambda^{2}}{4} \mathbb{E}_{B i n(n, 4 R)}\left[e^{\lambda Z} \sum_{\ell=1}^{n} e^{\lambda . I_{\ell}\left(g_{R}^{\eta}\right)} I_{\ell}^{2}\left(g_{R}^{\eta}\right) \mathbb{1}_{\mathcal{A}^{c}}\right] \leq \frac{\lambda^{2}}{4} e^{-\frac{\gamma}{3}(\log n)^{k}+\log n}
$$

for all $n \geq M_{1}$. Since $\mathbb{E}_{\operatorname{Bin}(n, 4 R)}\left[e^{\lambda Z}\right] \geq 1$ :

$\mathbb{E}_{\operatorname{Bin}(n, 4 R)}\left[e^{\lambda Z}\right]-\mathbb{E}_{\operatorname{Bin}(n, 4 R)}^{2}\left[e^{\frac{\lambda}{2} Z}\right] \leq \mathbb{E}_{\operatorname{Bin}(n, 4 R)}\left[e^{\lambda Z}\right]\left(\frac{e \lambda^{2}}{4 n^{\varepsilon}}+\frac{\lambda}{4} e^{\frac{-\gamma}{3}(\log n)^{k}+\log n}\right)$

Note that there exists $M_{2}(\varepsilon, \gamma, k)$ (the worst case is when $\left.\lambda=\frac{\gamma}{3}(\log n)^{k}\right)$ such that

$$
\frac{e \lambda^{2}}{4 n^{\varepsilon}}+\frac{\lambda}{4} e^{-\frac{\gamma}{3}(\log n)^{k}+\log n}<\frac{100}{\gamma^{2}(\log n)^{2 k}}
$$

for all $n \geq M_{2}$. Let $M_{3}=\max \left\{M_{1}, M_{2}\right\}$. Then:

$$
\mathbb{E}_{\operatorname{Bin}(n, 4 R)}\left[e^{\lambda Z}\right]-\mathbb{E}_{\operatorname{Bin}(n, 4 R)}^{2}\left[e^{\frac{\lambda}{2} Z}\right] \leq \frac{\mathbb{E}_{\operatorname{Bin}(n, 4 R)}\left[e^{\lambda Z}\right] \cdot 100 \lambda^{2}}{\gamma^{2}(\log n)^{2 k}}
$$

for all $n \geq M_{3}$ and $0<\lambda<\frac{\gamma}{3}(\log n)^{k}$. Now we define $F:[0,+\infty) \rightarrow \mathbb{R}$

$$
F(\lambda)= \begin{cases}\mathbb{E}_{\operatorname{Bin}(n, 4 R)}\left[e^{\lambda(Z-\mathbb{E} Z)}\right], & \text { if } 0 \leq \lambda \leq \frac{\gamma}{3}(\log n)^{k}, \\ 0 & \text { if } \lambda \geq \frac{\gamma}{3}(\log n)^{k}\end{cases}
$$

Note that

$$
\left(1-\frac{100 \lambda^{2}}{\gamma^{2}(\log n)^{2 k}}\right) F(\lambda) \leq(F(\lambda / 2))^{2}
$$

Now, we consider $G(x):=F\left(\frac{\gamma(\log n)^{k}}{10} x\right)$ for every $x \in(0,1)$ and observe that the following properties of $G$ hold.

First, note that $G(0)=1$ and $G^{\prime}(0)=0$, since it satisfies the conditions of Leibniz's Rule. Next we have that:

$$
\left(1-x^{2}\right) G(x) \leq G(x / 2)^{2}
$$

for every $x \in(0,1)$.

We may now apply this Lemma 7.16 to get that for every $\lambda \in$ $\left(0, \frac{\gamma}{10}(\log n)^{k}\right)$ 


$$
F(\lambda) \leq\left(1-\frac{100 \lambda^{2}}{\gamma^{2}(\log n)^{2 k}}\right)^{-2}
$$

Pick $\lambda_{0}=\frac{\gamma(\log n)^{k}}{20}$. Then $F\left(\lambda_{0}\right) \leq 2$ for every $n \geq M_{3}$.

Finally, we let $H(x):=e^{x \frac{\gamma}{20}(\log n)^{k}}$. By Markov's Inequality we have that for every $t>0$ :

$$
\begin{aligned}
\mathbb{P}_{B i n(n, 4 R)}\left(Z-\mathbb{E}_{B i n(n, 4 R)}[Z] \geq t\right) & \leq \frac{\mathbb{E}\left[H\left(Z-\mathbb{E}_{\operatorname{Bin}(n, 4 R)}[Z]\right)\right]}{H(t)} \\
& \leq 2 . e^{-t \frac{\gamma}{20}(\log n)^{k}}
\end{aligned}
$$

Since for any probability measure

$$
\mathbb{P}(|Z-\mathbb{E}[Z]| \geq t) \leq \mathbb{P}(Z-\mathbb{E}[Z] \geq t)+\mathbb{P}(Z-\mathbb{E}[Z] \leq-t),
$$

and by the Inequality (9), we may conclude by duality that

$$
\mathbb{P}_{(\operatorname{Bin}(n, 4 R)}\left(\left|Z-\mathbb{E}_{\operatorname{Bin}(n, 4 R)}[Z]\right| \geq t\right) \leq 4 . e^{-t \frac{\gamma}{20}(\log n)^{k}}
$$

for every $n \geq M_{3}$. Let $t>0$ be a fixed constant and pick up $M_{4}(\gamma, t)$ such that

$$
\text { 4. } e^{-t \frac{\gamma}{20}(\log n)^{k}} \leq e^{-t \frac{\gamma}{100}(\log n)^{k}} .
$$

So, we get that for $N_{2}=\max \left\{M_{3}, M_{4}(\gamma, t)\right\}$

$$
\mathbb{P}_{\operatorname{Bin}(n, 4 R)}\left(\left|Z-\mathbb{E}_{\operatorname{Bin}(n, 4 R)}[Z]\right| \geq t\right) \leq e^{-t \frac{\gamma}{100}(\log n)^{k}}
$$

for every $n \geq N_{2}(t, \gamma, \varepsilon, k):=\max \left\{M_{1}, M_{2}(\varepsilon, \gamma, k), M_{4}(\gamma, t)\right\}$.

The reader may note that the only fact that we used from $\mathbb{P}_{\operatorname{Bin}(n, 4 R)}$ is that it has a fixed number of points. So we may state the following theorem for $\mathbb{P}_{\operatorname{Bin}\left(n, 4 R^{*}\right)}$ and the proof will carry out in the exact same way.

Theorem 7.17 Let $R$ be an axis parallel rectangle and denote by $R^{\prime}$ its rotation by $\pi / 2$. Suppose that there exists $k \geq 1, M_{1} \in \mathbb{N}, \gamma>0$ and $\varepsilon>0$ such that

$$
\begin{array}{r}
\mathbb{P}_{\operatorname{Bin}\left(n, 4 R^{*}\right)}\left(\sum_{\ell=1}^{n} I_{\ell}^{2}\left(h_{R}^{\eta}\right) \geq n^{-\varepsilon}\right) \leq e^{-\gamma(\log n)^{k}} \\
\mathbb{P}_{\operatorname{Bin}\left(n, 4 R^{\prime *}\right)}\left(\sum_{\ell=1}^{n} I_{\ell}^{2}\left(h_{R^{\prime}}^{\eta}\right) \geq n^{-\varepsilon}\right) \leq e^{-\gamma(\log n)^{k}}
\end{array}
$$


for all $n \geq M_{1}$. Then, for any $t>0$, there exists $N_{2}(t, \gamma, \varepsilon, k)$ such that:

$$
\mathbb{P}_{\operatorname{Bin}\left(n, 4 R^{*}\right)}\left(\left|\mathbb{P}\left(H_{R} \mid \eta\right)-\mathbb{P}_{\operatorname{Bin}\left(n, 4 R^{*}\right)}\left(H_{R}\right)\right| \geq t\right) \leq e^{-t(\log n)^{k} \frac{\gamma}{100}}
$$

for all $n \geq N_{2}$.

\section{4}

\section{Proof of the Main Theorem for the auxiliary models}

In this section we prove Theorem 7.2. In fact we shall prove a version which is more explicit about the value of $N^{(k)}$. We show that we may take $N^{(k)}=N^{8^{k-1}}$, a double exponential function, where the base $N$, is a constant which depends only on $\theta$ and $\tau$.

Theorem 7.18 There exists a sequence $\gamma_{k}$ of positive real numbers such that the following holds: Let $R^{0}$ be an axis-parallel rectangle with bounded aspect ratio $\rho \in(\theta, 1 / \theta)$ (with $\theta \leq 1 / 3)$. For each $k \geq 2$ and for every $t \in[\tau, \infty)$ (with $\tau \leq \psi / 2$, where $\psi$ is a constant defined in (13))

$$
\mathbb{P}_{B i n\left(n, 4 R^{0}\right)}\left(\left|\mathbb{P}\left(H_{R^{0}} \mid \eta\right)-\mathbb{P}_{B i n\left(n, 4 R^{0}\right)}\left(H_{R^{0}}\right)\right| \geq t\right) \leq e^{-t \gamma_{k}(\log n)^{k}},
$$

for all $n \geq N^{8^{k-1}}$, where $N=N(\theta, \tau)$ is the same constant defined in (14).

Imposing that $\theta \leq 1 / 3$ and $\tau \leq \psi / 2$ loses no generality, since those are the harder cases that includes when we make no demands on $\theta$ and $\tau$. From now on Just as we did in the previous sections, we will do it for the model $\mathbb{P}_{\operatorname{Bin}(n, 4 R)}$ and observe that an analogous proof will give us the same theorem for $\mathbb{P}_{B i n\left(n, 4 R^{*}\right)}$.

This result is proved by induction. To prove the base case $k=2$ we require a result at the $k=1$ level. This result, which we state as Lemma 7.19, is essentially just a version of Theorem 6.2 for the $\mathbb{P}_{B i n(n, 4 R)}$ model. Since the result is very close to that theorem we simply sketch the proof.

Lemma 7.19 For all $\theta \in(0,1 / 2)$, there exists $M(\theta) \geq 1$ and $1 / 4>\gamma>0$ such that the following holds. Let $R^{0}$ be an axis-parallel rectangle with aspect ratio $\rho \in(\theta, 1 / \theta)$. Then

$$
\mathbb{P}_{\operatorname{Bin}\left(n, 4 R^{0}\right)}\left(\left|\mathbb{P}\left(H_{R^{0}} \mid \eta\right)-\mathbb{P}_{\operatorname{Bin}\left(n, 4 R^{0}\right)}\left(H_{R^{0}}\right)\right| \geq n^{-\gamma}\right) \leq n^{-\gamma}
$$

for every $n \geq M$.

Proof.[Sketch of the proof] It will follow essentially from the following three basic facts: 
RSW Theorem for the $\mathbb{P}_{B i n(n, 4 R)}$ model: Observe that as a consequence of the RSW Theorem for annealed Voronoi Percolation and Lemma 6.24 we have that the RSW Theorem for the $\mathbb{P}_{\operatorname{Bin}(n, 4 R)}$ model. In particular, if we pick the Rectangle $R_{1}$ of aspect ratio $3: 1$, we have that there exists $\psi>0$ such that for all $n$

$$
\psi<\mathbb{P}_{\operatorname{Bin}\left(n, 4 R^{1}\right)}\left(H_{R^{1}}\right)<1-\psi .
$$

Weaker bound for the Poisson model: That is, we have by Theorem 6.5 that there exists $\phi$ depending only on the aspect ratio of $R^{1}$ (which is a fixed constant 3), such that

$$
\mathbb{P}_{P o}\left(\mathbb{P}\left(H_{R^{1}} \mid \eta\right) \leq \frac{1}{2^{k}}\right) \leq(1-\phi)^{k}
$$

for all sufficiently large $k \in \mathbb{N}$.

And finally, a version of Corollary 6.17 for the $\mathbb{P}_{B i n(n, 4 R)}$ model: Indeed, the same proof gives us:

Corollary 7.20 For every rectangle $R$, let $a(n)=\mathbb{E}\left[\sum_{m=1}^{n} I_{m}\left(g_{R}^{\eta}\right)^{2}\right]$. Then

$$
\mathbb{P}_{\operatorname{Bin}(n, 4 R)}\left(\left|\mathbb{P}\left(H_{R} \mid \eta\right)-\mathbb{P}_{\operatorname{Bin}(n, 4 R)}\left(H_{R}\right)\right| \geq a(n)^{1 / 3}\right) \leq a(n)^{1 / 3}
$$

With those, the only difference for the proof of Theorem 6.2 is that we will define an algorithm to decide $g_{R}^{\eta}$ just as we did Chapter 7.1. We expect the readers to convince themselves of that.

We will apply Lemma 7.19 for $\theta=1 / 3$. So, it works for $R^{1}$ the rectangle of aspect ratio $3: 1$. So, there exists $M(1 / 3)$ such that:

$$
\mathbb{P}_{\operatorname{Bin}\left(n, 4 R^{1}\right)}\left(\left|\mathbb{P}\left(H_{R^{1}} \mid \eta\right)-\mathbb{P}_{\operatorname{Bin}\left(n, 4 R^{1}\right)}\left(H_{R^{1}}\right)\right| \geq n^{-\gamma}\right) \leq n^{-\gamma}
$$

for every $n \geq M$.

We may now define the constant $N=N(\theta, \tau)$ included in the statement of Theorem 7.18. We will also be interest in picking up $\theta \leq 1 / 3$ so it contains the ratio of the rectangle $R^{1}$. We recall that $\psi$ is defined in (13). Set $\varepsilon(\psi / 2)>0$ as in Theorem 7.8. Let $N(\theta, \tau)$ be defined as the smallest integer such that

$$
\log (N) \geq 100|\log (\theta)|+(400 / \psi)^{8}+1 / \tau^{8}+(400 / \gamma)^{8}+\left(1 / \varepsilon^{4}\right)^{1 / \varepsilon^{4}}+M
$$

Now note that for every $n \geq N$ such that $\psi / 2>n^{-\gamma}$ for all $n \geq N$. So, we have that for $R^{1}$

$$
\mathbb{P}_{\operatorname{Bin}\left(n, 4 R^{1}\right)}\left(\frac{\psi}{2}<\mathbb{P}\left(H_{R^{1}} \mid \eta\right)<1-\frac{\psi}{2}\right) \geq 1-e^{-\gamma \log n}
$$


for all $n \geq N$.

Define the events $E^{(1)}:=\left\{\frac{\psi}{2}<\mathbb{P}\left(H_{R^{1}} \mid \eta\right)<1-\frac{\psi}{2}\right\}$ and $E^{(2)}:=\{$ no point outside $2 R^{1}$ interferes in the event $\left.E^{(1)}\right\}$. Set $E:=E^{(1)} \cap E^{(2)}$ and observe that both $E \in \mathcal{F}_{2 R^{1}}$ and

$$
\mathbb{P}_{\operatorname{Bin}\left(n, 4 R^{1}\right)}(E) \geq 1-e^{-\frac{\gamma}{2} \log n},
$$

for all $n \geq N$. Consider $R_{n}^{1}$. In particular, we may renormalize the event $E$, to an event $E_{n}:=E_{n}^{(1)} \cap E_{n}^{(2)}$, where $E_{n}^{(1)}:=\left\{\frac{\psi}{2}<\mathbb{P}\left(H_{R_{n}^{1}} \mid \eta\right)<1-\frac{\psi}{2}\right\}$ and $E^{(2)}:=\left\{\right.$ no point outside $2 R_{n}^{1}$ interferes in the event $\left.E_{n}^{(1)}\right\}$. Since, we have that

$$
\mathbb{P}_{\operatorname{Bin}\left(m, 4 R^{1}\right)}\left(E^{c}\right) \leq e^{-\frac{\gamma}{2} \log m}
$$

for all $m \geq N$, by the properties of the binomial distribution, the same applies to the renormalized rectangle

$$
\mathbb{P}_{\operatorname{Bin}\left(m, 4 R_{n}^{1}\right)}\left(E_{n}^{c}\right) \leq e^{-\frac{\gamma}{2} \log m}
$$

for all $m \geq N$. From which it follows that

$$
\mathbb{P}_{\operatorname{Bin}\left(m, 4 R_{n}^{1}\right)}\left(E_{n}^{c}\right) \leq e^{-\frac{\gamma}{2} \log n}
$$

for all $m \geq n$, whenever $n \geq N$.

We may now finally use the comparison from $\mathbb{P}_{\operatorname{Bin}\left(n, 4 R_{n}^{1}\right)}$ to $\mathbb{P}_{\text {Po }}$. Applying Corollary 7.6 we obtain

$$
\mathbb{P}_{\text {Po }}\left(\frac{\psi}{2}<\mathbb{P}\left(H_{R_{n}^{1}} \mid \eta\right)<1-\frac{\psi}{2}\right) \geq 1-e^{-\frac{\gamma}{4} \log n},
$$

for all $n \geq N(\theta, \tau)$.

Proof.[Proof of Theorem 7.18] We will prove this result by induction:

Base Case $k=2$ : Our approach will be based on Theorem 7.8 and Theorem 7.14. Let $R^{0}$ be a rectangle of bounded aspect ratio $\rho \in(\theta, 1 / \theta)$. By the conditions satisfied in Inequality (15), we can use Theorem 7.8 to deduce that there exists $\varepsilon(\psi)$ (the same that we picked before) such that for all $R \in\left(R^{0}, R^{0 \prime}, R^{1}, R^{1 \prime}\right)$,

$$
\mathbb{P}_{B i n\left(n, 4 R^{0}\right)}\left(\sum_{\ell=1}^{n} I_{\ell}^{2}\left(g_{R^{0}}^{\eta}\right) \geq n^{-\varepsilon}\right) \leq e^{-\frac{\gamma}{400}(\log n)^{2}}
$$

for all $n \geq M_{1}$. We remind the reader that the constant $M_{1}$ of Theorem 7.8 may be taken to be $M_{1}=\max \left\{N^{8}, N(\gamma, 1), N^{*}(\gamma, \theta), N^{* *}(\gamma), N^{* * *}(\gamma)\right\}$, where $N(\gamma, 1), N^{*}(\gamma, \theta), N^{* *}(\gamma)$ and $N^{* * *}(\gamma)$ are the functions defined in the proofs of Proposition 7.9 and Lemma 7.12 respectively. It is easily checked, with the value of $N$ we have chosen (14) that the maximum is obtained by $N^{8}$. And so we have(16) for all $n \geq M_{1}=N^{8}$. 
With that, all the inequalities are satisfied by $N$ already, and so $N^{8}$ is by a large margin the maximum.

We may now use the condition given by (16), and with Theorem 7.14 we conclude that for any $t>0$ and $R \in\left(R^{0}, R^{0 \prime}, R^{1}, R^{1 \prime}\right)$,

$$
\mathbb{P}_{B i n(n, 4 R)}\left(\left|\mathbb{P}\left(H_{R} \mid \eta\right)-\mathbb{P}_{B i n(n, 4 R)}\left(H_{R}\right)\right| \geq t\right) \leq e^{-t(\log n)^{2} \frac{\gamma}{(200)^{2}}},
$$

for all $n \geq N_{2}$. We should again ask ourselves if we can decide the behavior of $N_{2}$. We remind the reader that $N_{2}$ of Theorem 7.14 may be taken to be that $N_{2}:=\max \left\{M_{1}, M_{2}(\varepsilon, \gamma, k), M_{4}(\gamma, t)\right\}$, where $M_{2}(\varepsilon, \gamma, k)$ and $M_{4}(\gamma, t)$ are the functions defined in the proof of Theorem 7.14. Remember that as we have seen, $M_{1}=N^{8}$. In fact, this again will be the chosen maximum. To observe this, it is useful that $N$ also satisfies that $\log (N) \geq\left(1 / \varepsilon^{4}\right)^{1 / \varepsilon^{4}}+1 / \tau^{8}+(400 / \gamma)^{8}$, when controlling $M_{2}$ and $M_{4}$. One may also note that the worst case when controlling $M_{4}$, given by Inequality (12), is when $t=\tau$. So, using simple inequalities together with the imposed conditions, one may conclude that $N_{2}=N(\theta, \tau)^{8}$ for every $t \in[\tau, \infty)$.

And so we have proved that for $R^{0}$ with bounded aspect ratio $\rho \in(\theta, 1 / \theta)$ (which includes the rectangle of aspect ratio $3: 1$ ) we have that for all $t \in[\tau, \infty)$

$$
\mathbb{P}_{\operatorname{Bin}\left(n, 4 R^{0}\right)}\left(\left|\mathbb{P}\left(H_{R^{0}} \mid \eta\right)-P_{\operatorname{Bin}\left(n, 4 R^{0}\right)}\left(H_{R^{0}}\right)\right| \geq t\right) \leq e^{-t(\log n)^{2} \frac{\gamma}{200^{2}}}
$$

for all $n \geq N^{(2)}=N^{8}$.

Applying this for $R^{1}$ and $t=\psi / 2$, which is perfectly fine since they belong to the intervals give us that:

$$
\mathbb{P}_{\text {Po }}\left(\frac{\psi}{2}<\mathbb{P}\left(H_{R_{n}^{1}} \mid \eta\right)<1-\frac{\psi}{2}\right) \geq 1-e^{-\frac{\psi}{2}(\log n)^{2} \frac{\gamma}{4(200)^{2}}}
$$

for all $n \geq N^{(2)}=: N^{8}$, where $N$ is the same as the one defined in (14). With that, we have a reasonable guess to our induction. That is:

Inductive step $k \geq 3$ : We think of $k$ as $j+1$ for some $j \geq 2$. So we may assume the following by the induction hypothesis. Let $R^{0}$ be an axis parallel rectangle with bounded aspect ratio $\rho \in(\theta, 1 / \theta)$ and $t \in[\tau, \infty)$ the following holds:

$$
\mathbb{P}_{\operatorname{Bin}\left(n, 4 R^{0}\right)}\left(\left|\mathbb{P}\left(H_{R^{0}} \mid \eta\right)-P_{\operatorname{Bin}\left(n, 4 R^{0}\right)}\left(H_{R^{0}}\right)\right| \geq t\right) \leq e^{-t\left(\frac{\psi}{2}\right)^{j-2}(\log n)^{j} \frac{\gamma}{(200)^{2(j-1)}}}
$$

for all $n \geq N^{(j)}=N^{8^{j-1}}$.

By arguing as above ( $k=2$ case) we obtain 


$$
\mathbb{P}_{\text {Po }}\left(\frac{\psi}{2}<\mathbb{P}\left(H_{R_{n}^{1}} \mid \eta\right)<1-\frac{\psi}{2}\right) \geq 1-e^{-\left(\frac{\psi}{2}\right)^{j-1}(\log n)^{j} \frac{\gamma}{4(200)^{2(j-1)}}}
$$

for all $n \geq N^{8^{j-1}}$.

With Inequality (17) and what was already done in the case $k=2$, the proof of the induction step it is fairly easy. One may note that by Theorem 7.8:

$$
\mathbb{P}_{\operatorname{Bin}\left(n, 4 R^{0}\right)}\left(\sum_{\ell=1}^{n} I_{\ell}^{2}\left(g_{R^{0}}^{\eta}\right) \geq n^{-\varepsilon}\right) \leq e^{-\left(\frac{\psi}{2}\right)^{j-1}(\log n)^{j+1} \frac{\gamma}{400(200)^{2(j-1)}}},
$$

for all $n \geq M_{1}$. Again, we ask ourselves what will be the behavior of $M_{1}$. Using now the hypothesis that $N$ also satisfies that $\log (N) \geq(400 / \psi)^{8}$, turns this easy with the same previous arguments. In fact, the growth of the exponent in $N^{8^{j-1}}$ is turning the inequalities even easier, even though the exponent on $\alpha(\log n)^{j}$ is getting bigger and the constant $\alpha$ is getting smaller. With simple computations we may conclude that $M_{1}=\left(N^{8^{j-1}}\right)^{8}=N^{8^{j}}$.

We will now use Theorem 7.14 to conclude that

$$
\mathbb{P}_{B i n(n, 4 R)}\left(\left|\mathbb{P}\left(H_{R} \mid \eta\right)-\mathbb{P}_{\operatorname{Bin}(n, 4 R)}\left(H_{R}\right)\right| \geq t\right) \leq e^{-t\left(\frac{\psi}{2}\right)^{j-1}(\log n)^{j+1} \frac{\gamma}{(200)^{2 j}}}
$$

for all $n \geq N_{2}$. Again $N_{2}=N^{8^{j}}$. Then, we conclude the induction step, thus, finishing the proof. We left the remaining details to the reader.

Now that we have proved it we finally observe that the same result is valid for the $\mathbb{P}_{\operatorname{Bin}\left(n, 4 R^{*}\right)}$ (maybe with different constants, but just pick the worst !)

Theorem 7.21 Let $R^{0}$ be an axis-parallel rectangle with bounded aspect ratio $\rho \in(\theta, 1 / \theta)$. For each $k \geq 2$, the following holds: For every $t \in[\tau, \infty)$

$$
\mathbb{P}_{B i n\left(n, 4 R^{0 *}\right)}\left(\left|\mathbb{P}\left(H_{R^{0}} \mid \eta\right)-P_{B i n\left(n, 4 R^{0 *}\right)}\left(H_{R^{0}}\right)\right| \geq t\right) \leq e^{-t\left(\frac{\psi}{2}\right)^{k-2}(\log n)^{k} \frac{\gamma}{200^{2(k-1)}}}
$$

for all $n \geq N^{(k)}(\theta, \tau)=N^{8^{k-1}}$.

This comes with the exact same proof as we did for the $\mathbb{P}_{\operatorname{Bin}\left(n, 4 R^{0}\right)}$ case but substituting Lemma 7.6 for Lemma 7.7, Theorem 7.8 for Theorem 7.13, Theorem 7.8 for Theorem 7.17, and that $\mathbb{P}_{\operatorname{Bin}\left(n, 4 R^{1 *}\right)}\left(H_{R^{1}}\right)$ is in the interval $(\psi, 1-\psi)$ for every $n$, which comes from Lemma 6.25 instead of 6.24 . We also get as a of the proof that for all $k \geq 1$

$$
\mathbb{P}_{P o \mid \mathbb{H}}\left(\frac{\psi}{2}<\mathbb{P}\left(H_{R_{n}^{1}} \mid \eta\right)<1-\frac{\psi}{2}\right) \geq 1-e^{-\left(\frac{\psi}{2}\right)^{k-1}(\log n)^{k} \frac{\gamma}{4(200)^{2(k-1)}}}
$$


for all $n \geq N^{8^{k-1}}$. With this fact, that will be fundamental in the proof of our final result, we end this chapter.

\section{5}

\section{Final remarks to achieve the improvement on the original model}

In this final section we will turn ourselves back to the original model $\mathbb{P}_{\operatorname{Bin}(n, R)}$ so we can achieve the improved bound for it. We will also do two corollaries of this improved bound. This result will come from three facts. The first two were proven in the last section, and we now state them as theorems. Consider $\gamma_{k}:=\left(\frac{\psi}{2}\right)^{k-1} \frac{\gamma}{4(200)^{2(k-1)}}$. So

Theorem 7.22 Let $R^{1}$ be the rectangle of aspect ratio $3: 1$. So, for every $k \geq 1$ we have that

$$
\mathbb{P}_{\text {Po }}\left(\frac{\psi}{2}<\mathbb{P}\left(H_{R_{n}^{1}} \mid \eta\right)<1-\frac{\psi}{2}\right) \geq 1-e^{-\gamma_{k}(\log n)^{k}}
$$

for all $n \geq N^{8^{k-1}}$.

Theorem 7.23 Let $R^{1}$ be the rectangle of aspect ratio $3: 1$. So, for every $k \geq 1$ we have that

$$
\mathbb{P}_{P o \mid \mathbb{H}}\left(\frac{\psi}{2}<\mathbb{P}\left(H_{R_{n}^{1}} \mid \eta\right)<1-\frac{\psi}{2}\right) \geq 1-e^{-\gamma_{k}(\log n)^{k}}
$$

for all $n \geq N^{8^{k-1}}$.

The other important fact is that similarly to the fact that we can translate Corollary 6.17 to the $\mathbb{P}_{\operatorname{Bin}(n, 4 R)}$ model, we can also translate Theorem 7.14 to a version for the original binomial model $\mathbb{P}_{\operatorname{Bin}(n, R)}$, without any change in the proof.

Theorem 7.24 Let $R$ be an axis parallel rectangle rectangle and denote by $R^{\prime}$ its rotation by $\pi / 2$. Suppose that there exists $k \geq 2, M_{1} \in \mathbb{N}, \gamma>0$ and $\varepsilon>0$ such that

$$
\begin{gathered}
\mathbb{P}_{\operatorname{Bin}(n, R)}\left(\sum_{\ell=1}^{n} I_{\ell}^{2}\left(f_{R}^{\eta}\right) \geq n^{-\varepsilon}\right) \leq e^{-\gamma(\log n)^{k}} \\
\mathbb{P}_{\operatorname{Bin}\left(n, R^{\prime}\right)}\left(\sum_{\ell=1}^{n} I_{\ell}^{2}\left(f_{R^{\prime}}^{\eta}\right) \geq n^{-\varepsilon}\right) \leq e^{-\gamma(\log n)^{k}}
\end{gathered}
$$

for all $n \geq M_{1}$. Then, for any $t>0$, there exists $N_{2}(t, \gamma, \varepsilon, k)$ such that:

$$
\mathbb{P}_{\operatorname{Bin}(n, 4 R)}\left(\left|\mathbb{P}\left(H_{R} \mid \eta\right)-\mathbb{P}_{\operatorname{Bin}(n, 4 R)}\left(H_{R}\right)\right| \geq t\right) \leq e^{-t(\log n)^{k} \frac{\gamma}{100}}
$$


for all $n \geq N_{2}$.

With those 2 Theorems, we will prove Theorem 7.1. More specifically

Theorem 7.25 Let $R^{0}$ be an axis-parallel rectangle with bounded aspect ratio $\rho \in(\theta, 1 / \theta)$. For each $k \geq 1$, the following holds: For every $t \in[\tau, \infty)$

$$
\mathbb{P}_{\operatorname{Bin}\left(n, R^{0}\right)}\left(\left|\mathbb{P}\left(H_{R^{0}} \mid \eta\right)-P_{\operatorname{Bin}\left(n, R^{0}\right)}\left(H_{R^{0}}\right)\right| \geq t\right) \leq e^{-t(\log n)^{k+1} \frac{\gamma_{k}}{(200)^{2}}}
$$

for all $n \geq N^{8^{k}}$.

Proof. With Theorems 7.22 and 7.23 we can produce a similar proof of Theorem 7.8 for the Binomial model for each $k \geq 1$. The only difference is that we will have to break in 3 cases just as we did in the proof of Theorem 6.2. This will make the constant a little bit worse, since in the first case we can only use that $|J| \geq \log n / 90$. But this won't be a major problem. In fact, we can guarantee that for all $R^{0}$ with bounded aspect ratio $\rho \in(\theta, 1 / \theta)$ there exists $\varepsilon(\psi)$ (the same as before) such that

$$
\mathbb{P}_{B i n\left(n, R^{0}\right)}\left(\sum_{\ell=1}^{n} I_{\ell}^{2}\left(f_{R^{0}}^{\eta}\right) \geq n^{-\varepsilon}\right) \leq e^{-(\log n)^{k+1} \frac{\gamma_{k}}{200}}
$$

for all $n \geq\left(N^{8^{k-1}}\right)^{8}$ (by the same reasons as before). With Theorem 7.24 we can finally conclude that for all $k \geq 1$ and $R^{0} \in(\rho, 1 / \rho)$ and $t \in[\tau, \infty)$ :

$$
\mathbb{P}_{\operatorname{Bin}\left(n, R^{0}\right)}\left(\left|\mathbb{P}\left(H_{R^{0}} \mid \eta\right)-P_{\operatorname{Bin}\left(n, R^{0}\right)}\left(H_{R^{0}}\right)\right| \geq t\right) \leq e^{-t(\log n)^{k+1} \frac{\gamma_{k}}{(200)^{2}}},
$$

for all $n \geq N^{8^{k}}$.

We can now derive from that, the following two corollaries:

Corollary 7.26 There exists $N=N(\theta, \tau)$ such that the following holds. Let $R^{0}$ be an axis-parallel rectangle with aspect ratio $\rho \in(\theta, 1 / \theta)$. Then for every $t \in[\tau, \infty)$

$$
\mathbb{P}_{\operatorname{Bin}\left(n, R^{0}\right)}\left(\left|\mathbb{P}\left(H_{R^{0}} \mid \eta\right)-\mathbb{P}_{\operatorname{Bin}\left(n, R^{0}\right)}\left(H_{R^{0}}\right)\right| \geq t\right) \leq e^{-e^{(\log \log n)^{2} / 20}},
$$

for every $n \geq N$.

Proof. Let $\tau>0$ be fixed. Consider $R^{0}$ in a collection of rectangles with bounded aspect ratio $\rho \in(\theta, 1 / \theta)$. By the choice of $N(\theta, \tau)$ and the behavior of $\gamma_{k}$ we have by Theorem 7.25 that 


$$
\mathbb{P}_{\operatorname{Bin}\left(n, R^{0}\right)}\left(\left|\mathbb{P}\left(H_{R^{0}} \mid \eta\right)-\mathbb{P}_{\operatorname{Bin}\left(n, R^{0}\right)}\left(H_{R}\right)\right| \geq t\right) \leq e^{-t(\log n)^{k+1} \frac{\gamma_{k}}{(200)^{2}}} \leq e^{-(\log n)^{k / 2}}
$$

for all $n \geq N^{8^{k}}$. Now pick $N^{*}(\theta, \tau)$ such that:

$$
\log \log n \geq 2 \log \log N
$$

for all $n \geq N^{*}$. We will show that

$$
\mathbb{P}_{\operatorname{Bin}\left(n, R^{0}\right)}\left(\left|\mathbb{P}\left(H_{R^{0}} \mid \eta\right)-\mathbb{P}_{\operatorname{Bin}\left(n, R^{0}\right)}\left(H_{R^{0}}\right)\right| \geq t\right) \leq e^{-\log n^{(\log (\log n)) / 20}}
$$

for all $n \geq N^{*}$.

Choose the maximum $k(n)$ such that $n \geq N^{8^{k}}$. Since $n<N^{8^{k+1}}$, we have by the Inequality (21) that for all $n \geq N^{*}$

$$
k \geq \log \log n / 10
$$

With that, we can conclude that

$$
e^{-(\log n)^{k / 2}} \leq e^{-(\log n)^{\log \log n / 20}}
$$

for all $n \geq N^{*}$. By the Inequality (20) we finally have

$$
\mathbb{P}_{\operatorname{Bin}_{n, R}}\left(\left|\mathbb{P}\left(H_{R} \mid \eta\right)-\mathbb{P}\left(H_{R}\right)\right| \geq t\right) \leq e^{-\log n^{(\log (\log n)) / 20}}=e^{-e^{(\log (\log n))^{2} / 20}}
$$

for all $n \geq N^{*}$, concluding our proof.

We can also prove the almost sure convergence result. We will get by the Borel-Cantelli Lemma that:

Corollary 7.27 For every rectangle $R^{0}$ with aspect ratio $\rho \in(\theta, 1 / \theta)$ :

$$
\mathbb{P}_{\operatorname{Bin}\left(n, R^{0}\right)}\left(\lim _{n \rightarrow \infty}\left|\mathbb{P}\left(H_{R^{0}} \mid \eta\right)-\mathbb{P}_{\operatorname{Bin}\left(n, R^{0}\right)}\left(H_{R^{0}}\right)\right|=0\right)=1
$$

We remark that we can't state that $\lim _{n \rightarrow \infty} \mathbb{P}\left(H_{R^{0}} \mid \eta\right)=\lim _{n \rightarrow \infty} P\left(H_{R^{0}}\right)$, since the convergence of crossing probabilities $\mathbb{P}\left(H_{R^{0}}\right)$ in Voronoi percolation remains open. .

Proof. Note that

$$
\begin{aligned}
& \mathbb{P}_{\operatorname{Bin}\left(n, R^{0}\right)}\left(\lim _{n \rightarrow \infty}\left|\mathbb{P}\left(H_{R^{0}} \mid \eta\right)-\mathbb{P}_{\operatorname{Bin}\left(n, R^{0}\right)}\left(H_{R^{0}}\right)\right|=0\right)= \\
& 1-\mathbb{P}_{\operatorname{Bin}\left(n, R^{0}\right)}\left(\bigcup_{j \geq 1} \limsup _{n \rightarrow \infty}\left|\mathbb{P}\left(H_{R^{0}} \mid \eta\right)-\mathbb{P}_{\operatorname{Bin}\left(n, R^{0}\right)}\left(H_{R^{0}}\right)\right|>1 / j\right)
\end{aligned}
$$


Now, let $j \in \mathbb{N}$ be fixed. Define $E_{n, j}:=\left\{\left|\mathbb{P}\left(H_{R^{0}} \mid \eta\right)-\mathbb{P}_{B i n\left(n, R^{0}\right)}\left(H_{R^{0}}\right)\right| \geq\right.$ $1 / j\}$. Note that by Theorem 7.25 (for $k=1$ ) we have that there exists $M=N(1 / j)$

$$
\mathbb{P}_{\operatorname{Bin}\left(n, R^{0}\right)}\left(\left|\mathbb{P}\left(H_{R^{0}} \mid \eta\right)-\mathbb{P}_{B i n\left(n, R^{0}\right)}\left(H_{R^{0}}\right)\right| \geq 1 / j\right) \leq e^{-a(\log n)^{2}}
$$

for all $n \geq N^{8}$. So, we have that for every fixed $j$ that $\sum_{n=1}^{\infty} \mathbb{P}_{\operatorname{Bin}_{n, R}}\left(E_{n, j}\right)<\infty$. By the first Borel-Cantelli Lemma (A.3), we have that

$$
\mathbb{P}_{B i n\left(n, R^{0}\right)}\left(\limsup _{n \rightarrow \infty} E_{n, j}\right)=0
$$

Finally, we may use the union bound to conclude that

$\mathbb{P}_{\operatorname{Bin}\left(n, R^{0}\right)}\left(\lim _{n \rightarrow \infty}\left|\mathbb{P}\left(H_{R^{0}} \mid \eta\right)-P\left(H_{R^{0}}\right)\right|=0\right) \geq 1-\sum_{j=1}^{\infty} \mathbb{P}_{B i n\left(n, R^{0}\right)}\left(\limsup _{n \rightarrow \infty} E_{n, j}\right)=1$

and we finish our proof.

With that, we have finally reached the end of this journey. I would be very happy to talk with interested people in this area. Percolation and Noise Sensitivity Theory are both new and amazing fields of mathematics. I would also like to thank my advisor Simon Griffiths. If it wasn't for him, I would never be able to accomplish this. More than that, I would have quit mathematics. I also thank professor Daniel Ahlberg for his useful commentaries. I wish that we can start new projects soon.

We all walked through a long journey, that I hope it has just started. 


\section{Bibliography}

[1] AHLBERG, D.; GRIFFITHS, S.; MORRIS, R. ; TASSION, V.. Quenched voronoi percolation. Advances in Mathematics, 286:889 - 911, 2016.

[2] BENJAMINI, I.; KALAI, G. ; SCHRAMM, O.. Noise sensitivity of Boolean functions and applications to percolation. Inst. Hautes udes Sci. Publ. Math., (90):5-43 (2001), 1999.

[3] BOLLOBÁS, B.; RIORDAN, O.. The critical probability for random voronoi percolation in the plane is $1 / 2$. Probability Theory and Related Fields, 136(3):417-468, Dec 2005.

[4] BOLlOBÁS, B.; RIORDAN, O.. Percolation. Cambridge University Press, 2006.

[5] BOUCHERON, S.; LUGOSI, G. ; MASSART, P.. Concentration Inequalities: A nonasymptotic theory of independence. OUP Oxford, 01 2013.

[6] BROAdBent, S. R.; HAMMERSLEY, J. M.. Percolation processes: I. crystals and mazes. Mathematical Proceedings of the Cambridge Philosophical Society, 53(3):629-641, 1957.

[7] DURRETT, R.. Probability: Theory and Examples. Cambridge Series in Statistical and Probabilistic Mathematics. Cambridge University Press, 5 edition, 2019.

[8] FORTUIN, C. M.; KASTELEYN, P. W. ; GINIBRE, J.. Correlation inequalities on some partially ordered sets. Comm. Math. Phys., 22(2):89-103, 1971.

[9] GARBAN, C.; STEIF, J. E.. Noise Sensitivity of Boolean Functions and Percolation. Institute of Mathematical Statistics Textbooks. Cambridge University Press, 2014.

[10] GRIMMETT, G.. Percolation. Springer-Verlag, 1989.

[11] HARRIS, T. E.. A lower bound for the critical probability in a certain percolation process. Mathematical Proceedings of the Cambridge Philosophical Society, 56(1):13-20, 1960. 
[12] JOOSTEN, M.. Random fractals and scaling limits in percolation. 2007.

[13] MOSSEL, E.; O'DONNELL, R. ; OLESZKIEWICZ, K.. Noise stability of functions with low influences: invariance and optimality. Annals of Mathematics, 171 (2010):295-341, 2010.

[14] O'DONNELL, R.. Analysis of Boolean Functions. Cambridge University Press, 2014.

[15] SCHRAMM, O.; STEIF, J. E.. Quantitative noise sensitivity and exceptional times for percolation. Annals of Mathematics, 171 (2010):619-672, 2010.

[16] SMIRNOV, S.; WERNER, W.. Critical exponents for two-dimensional percolation. Math. Res. Lett., 8(5-6):729-744, 2001.

[17] STEIF, J. E.. A mini course on percolation theory. Jyväskylä lectures in mathematics, (3), 2011.

[18] TASSION, V.. Crossing probabilities for voronoi percolation. The Annals of Probability, 44(5):3385-3398, 2016.

[19] VORONOI, G.. Nouvelles applications des parametres continus a la theorie des formes quadratiques. premier memoire. sur quelques proprietes des formes quadratiques positives parfaites. Journal fur die reine und angewandte Mathematik, 133:97-178, 1908. 


\section{A}

\section{Classic Inequalities}

We state and prove some of the most used inequalities in this section. Since some of them are widely known and given in Basic Probability courses, we will just state them and refer to [7] for more details. In contrast, the less usual ones will be rigorously proved.

Theorem A.1 (Holder's Inequality) Let $(\Omega, \mathcal{F}, \mu)$ be a measure space, and let $p, q \in[0,1]$ with $1 / p+1 / q=1$. Then, for all $f, g$ real-valued measurable functions on $(\Omega, \mathcal{F}, \mu)$ :

$$
\|f g\|_{1} \leq\|f\|_{p}\|g\|_{q}
$$

\section{Theorem A.2 (Lebesgue Dominated Convergence Theorem) Let}

$\left(f_{n}\right)$ be a sequence of real-valued measurable functions on a measure space $(\Omega, \mathcal{F}, \mu)$. Suppose that $f_{n}(x) \rightarrow f(x), \mu$-almost surely. Suppose that there exists an integrable function $g$ such that $\left|f_{n}(x)\right| \leq g(x)$, for every $n$ and for almost every $x \in \Omega$. Then, $f$ is integrable and

$$
\lim _{n \rightarrow \infty} \int_{\Omega} f_{n} d \mu=\int_{\Omega} f d \mu
$$

Theorem A.3 (First Borel-Cantelli Lemma) Let $E_{1}, E_{2}, \ldots$ be a sequence of events in a probability space. If we have that

$$
\sum_{i=1}^{\infty} \mathbb{P}\left(E_{i}\right)<\infty
$$

then

$$
\mathbb{P}\left(\bigcap_{n=1}^{\infty} \bigcup_{k=n}^{\infty} E_{k}\right)=0
$$

Theorem A.4 (Second Borel-Cantelli Lemma) Let $E_{1}, E_{2}, \ldots$ be a sequence of independent events (it may also be weakened to pairwise independence) in a probability space. If

$$
\sum_{i=1}^{\infty} \mathbb{P}\left(E_{i}\right)=\infty
$$


then

$$
\mathbb{P}\left(\bigcap_{n=1}^{\infty} \bigcup_{k=n}^{\infty} E_{k}\right)=1
$$

Theorem A.5 (Markov's Inequality) If $g: \mathbb{R}^{+} \rightarrow \mathbb{R}^{+}$is a monotonically increasing function, $X$ is a random variable $a \geq 0$ and $g(a)>0$, then

$$
\mathbb{P}(|X| \geq a) \leq \frac{\mathbb{E}[g(|X|)]}{g(a)}
$$

Theorem A.6 (Chebyshev's Inequality) If $X$ is a random variable, $\mathbb{E}[X]$ if finite, and $\operatorname{Var}(X), a>0$, then

$$
\mathbb{P}(|X-E[X]| \geq a) \leq \frac{\operatorname{Var}(X)}{a^{2}}
$$

Theorem A.7 (Stirling's Approximation) We have that for all $n \geq 1$

$$
\sqrt{2 \pi} n^{n+\frac{1}{2}} e^{-n} \leq n ! \leq e n^{n+\frac{1}{2}} e^{-n}
$$

The first inequality to be proved is of fundamental importance to Percolation Theory. It was first proved by Harris in [11], and later extended to other contests by Fortuin, Kasteleyn, and Ginibre in [8]. As it is stated below, the reader shall note that it is both valid for our applications in Quenched Voronoi Percolation and Percolation on $\mathbb{Z}^{2}$. But first, we need to take a few definitions to a more general context. This was based in [10].

Definição A.8 Consider $\left(\Omega, \mathcal{F}, \mathbb{P}_{p}\right)$ a probability space. We take $\Omega=$ $\prod_{s \in S}\{-1,1\}$ where $S$ is finite or countably infinite, $\mathcal{F}$ is the $\sigma$-field generated by the cylinder sets of $\Omega$, and $\mathbb{P}_{p}$ is a product measure on $(\Omega, \mathcal{F})$ defined by :

$$
\mathbb{P}=\prod_{s \in S} \mu_{s}
$$

where $\mu_{s}$ is given by $\mu_{s}(\omega(s)=1)=p, \mu_{s}(\omega(s)=-1)=1-p$, for vectors $\omega=(\omega(s): s \in S) \in \Omega$ and $p \in[0,1]$.

Similar to what we have done before, we consider a real-valued random variable $X$ on $(\Omega, \mathcal{F})$ to be increasing if $X(\omega) \leq X\left(\omega^{\prime}\right)$ whenever $\omega \leq \omega^{\prime}$ ( i.e. $\omega_{i} \leq \omega_{i}^{\prime}$, for every $i \in S$ ). A is called an increasing event int $\mathcal{F}$ if $\mathbb{1}_{A}(\omega) \leq \mathbb{1}_{A}\left(\omega^{\prime}\right)$ for $\omega \leq \omega^{\prime}$. 
One may note that this do not depend upon the lattice structure of the percolation process. So, the following results will be valid for the Triangular Lattice, $\mathbb{Z}^{2}$, and even Quenched Voronoi Percolation (having in mind the fact that the Poisson Point Process generates a countably infinite number of points with probability 1).

This first theorem is rather intuitive, but will proved to get the reader used to the previous definition.

Theorem A.9 If $X$ is a real-valued increasing random variable on $(\Omega, \mathcal{F})$ then

$$
\mathbb{E}_{p_{1}}(X) \leq \mathbb{E}_{p_{2}}(X), \text { whenever } p_{1} \leq p_{2},
$$

so long as the mean value exist. If $A$ is an increasing event in $\mathcal{F}$ then

$$
\mathbb{P}_{p_{1}}(A) \leq P_{p_{2}}(A), \text { whenever } p_{1} \leq p_{2}
$$

Proof. Let the random variables $(Y(\omega): \omega \in \Omega)$ be independent and uniformly distributed on $[0,1]$. We write

$$
\eta_{p}(\omega)= \begin{cases}1 & Y(\omega)<p \\ -1 & \text { otherwise }\end{cases}
$$

If $p_{1} \leq p_{2}$ then $\eta_{p_{1}} \leq \eta_{p_{2}}$, and since $X$ is an increasing random variable on $(\Omega, \mathcal{F}) X\left(\eta_{p_{1}}\right) \leq X\left(\eta_{p_{2}}\right)$. So we may take expectation on both sides and conclude that $\mathbb{E}_{p_{1}}(X) \leq \mathbb{E}_{p_{2}}(X)$. The second part follows when we apply the first part to $X=\mathbb{1}_{A}$.

Now we are finally able to state the $F K G$ inequality.

Theorem A.10 (Classic FKG Inequality) If $X$ and $Y$ are both increasing (or both decreasing) bounded real-valued random variables, then

$$
\mathbb{E}_{p}(X Y) \geq \mathbb{E}_{p}(X) E_{p}(Y)
$$

If one of the random variables in increasing, and the other decreasing, we get the inverted inequality $\mathbb{E}_{p}(X Y) \leq \mathbb{E}_{p}(X) E_{p}(Y)$.

Proof. First we prove it for real-valued random variables $X$ and $Y$ which only depend on a finite number of states of $\Omega$. Then, we will lift this restriction.

Suppose then that $X$ and $Y$ are increasing real-valued random variables which depend only on the states of $\omega_{1}, \ldots, \omega_{n}$. We will prove this result by induction in $n$. 
Suppose that $n=1$. Then, by monotonicity of $X$ and $Y$ we have that for every pair $a_{1}, a_{2} \in \Omega$

$$
\left(X\left(a_{1}\right)-X\left(a_{2}\right)\right)\left(Y\left(a_{1}\right)-Y\left(a_{2}\right)\right) \geq 0 .
$$

Thus,

$$
\begin{aligned}
0 & \leq \sum_{a_{1}, a_{2}}\left(X\left(a_{1}\right)-X\left(a_{2}\right)\right)\left(Y\left(a_{1}\right)-Y\left(a_{2}\right)\right) \mathbb{P}_{p}\left(\omega=a_{1}\right) \mathbb{P}_{p}\left(\omega=a_{2}\right) \\
& =2\left(\mathbb{E}_{p}(X Y)-E_{p}(X) E_{p}(Y)\right) .
\end{aligned}
$$

Now suppose that the result is valid for all $n<k$ and that $X$ and $Y$ are increasing functions that only depend on the states of $\omega_{1}, \ldots, \omega_{k}$. Then

$$
\begin{aligned}
E_{p}(X Y) & =E_{p}\left(E_{p}\left(X Y \mid \omega_{1}, \ldots, \omega_{k-1}\right)\right) \\
& =E_{p}\left(E_{p}\left(X \mid \omega_{1}, \ldots, \omega_{k-1}\right) E_{p}\left(Y \mid \omega_{1}, \ldots, \omega_{k-1}\right)\right)
\end{aligned}
$$

since, for fixed $\omega_{1}, \ldots, \omega_{k-1}$, we have that $X$ and $Y$ are increasing in $\omega_{k}$, and so, we may apply the case $k=1$. We also have that $E_{p}\left(X \mid \omega_{1}, \ldots, \omega_{k-1}\right)$ is an increasing function in $\omega, \ldots, \omega_{k-1}$, and the same is valid for $Y$. So, by the induction hypothesis

$$
\begin{aligned}
E_{p}(X Y) & \left.=E_{p}\left(E_{p}\left(X \mid \omega_{1}, \ldots, \omega_{k-1}\right) E_{p}\left(Y \mid \omega_{1}, \ldots, \omega_{k-1}\right)\right)\right) \\
& \geq E_{p}\left(E_{p}\left(X \mid \omega_{1}, \ldots, \omega_{k-1}\right)\right) E_{p}\left(E_{p}\left(Y \mid \omega_{1}, \ldots, \omega_{k-1}\right)\right) \\
& =\mathbb{E}_{p}(X) E_{p}(Y) .
\end{aligned}
$$

and we have proved our desired induction. We may now lift the condition that $X$ and $Y$ depend only on finitely many elements of $\Omega$. Suppose, then that $X$ and $Y$ obey the conditions of the theorem. Let $\omega_{1}, \omega_{2}, \ldots$ be a fixed ordering of the elements of $\Omega_{n}$. Define $X_{n}:=\mathbb{E}_{p}\left(X \mid \omega_{1}, \ldots, \omega_{n}\right)$, and $\left.Y_{n}:=E_{p}\left(Y \mid \omega_{1}, \ldots, \omega_{n}\right)\right)$. For each $n, X_{n}$ and $Y_{n}$ are increasing functions of the states $\omega_{1}, \ldots, \omega_{n}$, and so we may apply the previous result to yield that

$$
\mathbb{E}_{p}\left(X_{n} Y_{n}\right) \geq E_{p}\left(X_{n}\right) E_{p}\left(Y_{n}\right)
$$

Now we may use the Martingale Convergence Theorem, stated in Section 2, since $X$ and $Y$ are both bounded, and $\omega_{1}, \ldots, \omega_{n}$ is a filtration, we get that

$$
X_{n} \rightarrow X, a . s,
$$


and

$$
Y_{n} \rightarrow Y, a . s
$$

So, by dominated convergence, the left-hand side converges to $\mathbb{E}_{p}(X Y)$, and we finally have that

$$
\mathbb{E}_{p}(X Y) \geq E_{p}(X) E_{p}(Y)
$$

concluding our proof.

It is immediate from this that if we take $x, y, z, w$ in $Z^{2}$, or $\mathbb{T}$, and even points in $\mathbb{R}^{2}$ for a fixed Voronoi Tiling, and define $A$ to be the event that there is a red path from $x$ to $y$, and $B$ the event that there is a blue path from $z$ to $w$. Then $\mathbb{P}(A \cap B) \geq P(A) . P(B)$. So this applications of the FKG Inequality are well formalized.

For the annealed Voronoi Percolation we will follow an argument from [4]. But first we need to formalize some previous notions to this context.

Definição A.11 Consider $\mathcal{P}$ to be the Poisson Process generated in the plane. Now, with the Voronoi Tessellation in mind, we color each tile red with probability $p$ and each tile blue with probability $1-p$. This will leave us with a partition in two processes $\left(\mathcal{P}^{+}, \mathcal{P}^{-}\right)$. Consider $E$ to be an increasing event, if for every configuration $\omega_{1}=\left(X_{1}^{+}, X_{1}^{-}\right)$in $E$ and every configuration $\omega_{2}=\left(X_{2}^{+}, X_{2}^{-}\right)$, with $X_{1}^{+} \subseteq X_{2}^{+}$and $X_{1}^{-} \supseteq X_{2}^{-}$, we have $\omega_{2} \in E$. This means that $E$ is preserved when adding red points, or deleting blue points. The definition of an increasing function $f\left(\mathcal{P}^{+}, \mathcal{P}^{-}\right)$follows the same as before.

Lemma A.12 (FKG for annealed Voronoi Percolation) Let $E_{i}=$ $E_{i}\left(\mathcal{P}^{+}, \mathcal{P}^{-}\right), i=1,2$, be two increasing events. Then for any $0 \leq p \leq 1$, we have that

$$
\mathbb{P}_{p}\left(E_{1} \cap E_{2}\right) \geq \mathbb{P}_{p}\left(E_{1}\right) \mathbb{P}_{p}\left(E_{2}\right),
$$

where $\mathbb{P}_{p}$ is the probability measure associated to the Poisson Process in the plane with p-red coloring as denoted before.

Proof. Fixing $\mathcal{P}^{-}$, for each $i$, gives us that $\mathbb{1}_{E_{i}}$ is increasing in $\mathcal{P}^{+}$. So by the standard FKG inequality

$$
\mathbb{E}_{p}\left(\mathbb{1}_{E_{1}} \mathbb{1}_{E} \mid \mathcal{P}^{-}\right) \geq \mathbb{E}_{p}\left(\mathbb{1}_{E_{1}} \mid \mathcal{P}^{-}\right) \mathbb{E}_{p}\left(\mathbb{1}_{E_{2}} \mid \mathcal{P}^{-}\right)
$$

for every $\mathcal{P}^{-}$. Taking, over $\mathcal{P}^{-}$, the expectation on both sides, we get that :

$$
\mathbb{E}_{p}\left(\mathbb{1}_{E_{1}} \mathbb{1}_{E_{2}}\right) \geq \mathbb{E}_{p}\left(\mathbb{E}_{p}\left(\mathbb{1}_{E_{1}} \mid \mathcal{P}^{-}\right) \mathbb{E}_{p}\left(\mathbb{1}_{E_{2}} \mid \mathcal{P}^{-}\right)\right)
$$


But the functions $\mathbb{E}_{p}\left(\mathbb{1}_{E_{i}} \mid \mathcal{P}^{-}\right)$are both decreasing in $\mathcal{P}^{-}$. So we may use the previous theorem again to finally get that:

$\mathbb{E}_{p}\left(\mathbb{E}_{p}\left(\mathbb{1}_{E_{1}} \mid \mathcal{P}^{-}\right) \mathbb{E}_{p}\left(\mathbb{1}_{E_{2}} \mid \mathcal{P}^{-}\right)\right) \geq \mathbb{E}_{p}\left(\mathbb{E}_{p}\left(\mathbb{1}_{E_{1}} \mid \mathcal{P}^{-}\right)\right) \mathbb{E}_{p}\left(\mathbb{E}_{p}\left(\mathbb{1}_{E_{2}} \mid \mathcal{P}^{-}\right)\right)=\mathbb{E}_{p}\left(\mathbb{1}_{E_{1}}\right) \mathbb{E}_{p}\left(\mathbb{1}_{E_{2}}\right)$

So we conclude our lemma.

Our next inequality will be the celebrated van der Berg's, Kesten's inequality. The way it is stated here, and the proof of it may be found in $[12]$.

Let $A$ and $B$ be two increasing events. For $\omega \in \Omega_{n}$ and a set $S \subseteq[n]$ define $\omega_{S}(x)=\omega(x)$ if $x \in S$ and $\omega_{S}(x)=-1$ otherwise. We define :

$$
A \circ B:=\left\{\omega \in \Omega_{n}: \exists S \subseteq\{1, \ldots, n\} \text { such that } \omega_{S} \in A \text { and } \omega_{[n] \backslash S} \in B\right\}
$$

\section{Theorem A.13 (The BK Inequality for annealed Voronoi Percolation)} Let $A$ and $B$ be two red-increasing events. Then

$$
\mathbb{P}_{\text {Po }}(A \circ B) \leq \mathbb{P}_{\text {Po }}(A) \cdot \mathbb{P}_{\text {Po }}(B)
$$

Now we will state and prove a very simple but useful inequality. This can be proved in any context that the FKG inequality is valid, since it is a direct application of it. We particularly use it in the context of Percolation on $\mathbb{Z}^{2}$ and annealed Voronoi Percolation on $\mathbb{R}^{2}$.

Theorem A.14 (The Square Root Trick) Let $A_{1}, \ldots, A_{n}$ be increasing events and write $A:=\mathbb{P}\left(A_{1} \cup \ldots \cup A_{n}\right) \geq p$. We have that

$$
\max _{1 \leq i \leq n} \mathbb{P}_{p}\left(A_{i}\right) \geq 1-\left(1-\mathbb{P}_{p}(A)\right)^{1 / n} .
$$

Proof. First note that

$$
\mathbb{P}_{p}(A)=1-\mathbb{P}_{p}\left(A_{1}^{c} \cap \ldots \cap A_{n}^{c}\right) .
$$

Now by the FKG inequality

$$
\begin{aligned}
\mathbb{P}_{p}(A) & \leq 1-\mathbb{P}_{p}\left(A_{1}^{c}\right) \ldots \mathbb{P}_{p}\left(A_{n}^{c}\right) \\
& \leq 1-\left(1-\max _{1 \leq i \leq n} \mathbb{P}_{p}\left(A_{i}\right)\right)^{n}
\end{aligned}
$$

and thus we conclude the proof. 